\title{
THÉORÈMES LIMITES AVEC POIDS POUR LES MARTINGALES VECTORIELLES
}

\author{
FAOUZi ChaABAne ${ }^{1}$ And FAÏZA MaAOUiA ${ }^{1}$
}

\begin{abstract}
We give limit theorems specifying weak and strong rates of convergence associated to a quadratic extension of the martingale almost-sure central limit theorem. Some typical examples are discussed to illustrate how to make use of them in statistic.

Résumé. On donne des théorèmes limites précisant les vitesses de convergence (en loi et au sens presque-sûr) associées à une extension quadratique du théorème de limite centrale presque-sûre pour les martingales discrètes vectorielles. Des exemples typiques illustrent l'usage qu'on peut en faire en statistique.
\end{abstract}

AMS Subject Classification. 60F05, 60F42, 60F15.

Reçu le 26 janvier 1999. Révisé le 30 juin 1999 et le 31 août 2000.

\section{INTRODUCTION}

\subsection{Motivation. Exemples}

Une approche générale du théorème de la limite centrale presque-sûre (TLCPS) pour les martingales vectorielles discrètes a été développée dans [5] et [6]. Elle se situe dans l'un des cadres habituels assurant la convergence en loi d'une martingale vectorielle convenablement normalisée vers une loi gaussienne ou un mélange de lois non nécessairement gaussiennes. Pour la commodité du lecteur, les résultats seront rappelés en annexe à la fin de ce travail.

Notre but ici est de les compléter par des théorèmes limites avec poids (loi forte des grands nombres, théorème de limite centrale, loi du logarithme itéré) associés de manière naturelle à une extension quadratique du TLCPS. Les deux exemples suivants sont éclairants quant à la signification précise de l'extension visée et de la transcription statistique qu'on peut en tirer.

Exemple 1. (Échantillon d'une loi sur $\mathbb{R}^{d}$ )

Soit $\left(X_{i}, i \in \mathbb{N}^{*}\right)$ une suite de v.a. $d$-dimensionnelles, indépendantes et identiquement distribuées selon une loi de moyenne $m$ et de covariance inversible $\Gamma$. Considérons les estimateurs empiriques de $m$ et $\Gamma$ :

$$
\widehat{m}_{n}=n^{-1} \sum_{i=1}^{n} X_{i}, \quad \widehat{\Gamma}_{n}=(n-1)^{-1} \sum_{i=1}^{n}\left(X_{i}-\widehat{m}_{n}\right) *\left(X_{i}-\widehat{m}_{n}\right)
$$

\footnotetext{
Keywords and phrases: Martingale vectorielle, théorème de la limite centrale presque-sûre, lois fortes quadratique et logarithmique des grands nombres, loi du logarithme itéré.

1 Équipe d'Analyse Stochastique et Modélisation Statistique, DGRST E07/C15, Faculté des Sciences de Bizerte, Tunisie ; e-mail: Faouzi.Chaabane@fsb.rnu.tn \& Faiza.Maaouia@fst.rnu.tn
} 
$\left({ }^{*} x\right.$ étant le transposé du vecteur $\left.x\right)$. La formulation statistique du TLCPS pour la suite $\left(X_{i}, i \in \mathbb{N}^{*}\right)$ est :

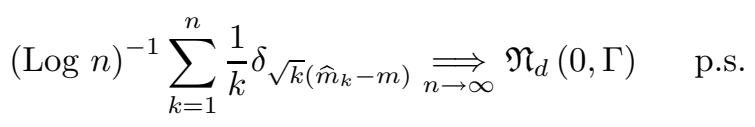

(i.e convergence étroite pour presque toutes les trajectoires des mesures empiriques logarithmiques associées à la suite $\left(\sqrt{n}\left(\widehat{m}_{n}-m\right)\right)$ vers la loi gaussienne $d$-dimensionnelle centrée et de covariance $\left.\Gamma\right)$. On en déduit que, hors d'un ensemble négligeable, l'inégalité suivante a lieu :

$$
\underline{\varliminf}(\log n)^{-1} \sum_{k=1}^{n}\left(\widehat{m}_{k}-m\right) *\left(\widehat{m}_{k}-m\right) \geq \Gamma
$$

au sens des matrices réelles symétriques semi-définies positives. En fait, on dispose du résultat plus précis :

$$
(\log n)^{-1} \sum_{k=1}^{n}\left(\widehat{m}_{k}-m\right) *\left(\widehat{m}_{k}-m\right) \underset{n \rightarrow \infty}{\longrightarrow} \Gamma \quad \text { p.s. }
$$

traduisant la convergence p.s. des moments du second ordre de la mesure empirique logarithmique considérée en (1.1) et appelé loi forte quadratique des grands nombres (LFQ). D'où l'on déduit les deux variantes significatives suivantes :

$$
\begin{gathered}
\widehat{\widehat{\Gamma}}_{n}=(\log n)^{-1} \sum_{k=1}^{n}\left(\widehat{m}_{k}-\widehat{m}_{n}\right) *\left(\widehat{m}_{k}-\widehat{m}_{n}\right) \underset{n \rightarrow \infty}{\longrightarrow} \Gamma \quad \text { p.s. } \\
\operatorname{tr}\left\{\Gamma^{-1 / 2} \widehat{\Gamma}_{n} \Gamma^{-1 / 2}\right\} \underset{n \rightarrow \infty}{\longrightarrow} d \quad \text { p.s. }
\end{gathered}
$$

Dans la suite, on montrera comment on peut préciser les vitesses de convergence (en loi ou au sens p.s.) pour de telles propriétés. En particulier, on peut établir grâce au théorème 2.5, que sous l'hypothèse supplémentaire $\mathbb{E}\left\{\left\|X_{1}\right\|^{2 \beta}\right\}<\infty$ pour un $\left.\left.\beta \in\right] 1,2\right]$, les deux alternatives gaussiennes suivantes au test classique de Whishart ont lieu :

$$
\begin{gathered}
\left\{\sqrt{\log n}\left[\widehat{\widehat{\Gamma}}_{n}-\Gamma\right], \sqrt{n}\left(\widehat{m}_{n}-m\right)\right\} \underset{n \rightarrow \infty}{\stackrel{\mathcal{L}}{\longrightarrow}} \mathfrak{N}_{d \times d}(0,2 \Sigma) \otimes \mathfrak{N}_{d}(0, \Gamma) \\
\left\{\frac{1}{2 \sqrt{d}} \sqrt{\log n}\left[\operatorname{tr}\left\{\Gamma^{-1 / 2} \widehat{\widehat{\Gamma}}_{n} \Gamma^{-1 / 2}\right\}-d\right], \sqrt{n}\left(\widehat{m}_{n}-m\right)\right\} \underset{n \rightarrow \infty}{\stackrel{\mathcal{L}}{\longrightarrow}} \mathfrak{N}(0,1) \otimes \mathfrak{N}_{d}(0, \Gamma)
\end{gathered}
$$

avec $\Sigma=\Gamma \otimes \Gamma+{ }^{\perp}\left(\operatorname{Vect}(\Gamma){ }^{*} \operatorname{Vect}(\Gamma)\right)(\otimes$ dénote le produit tensoriel de mesures ou de matrices ; Vect $(A)$ désigne le vecteur obtenu en empilant les vecteurs colonnes de la matrice $A$ et ${ }^{\perp}\left(\operatorname{Vect}(\Gamma){ }^{*} \operatorname{Vect}(\Gamma)\right)$ est la matrice à blocs dont le bloc d'indices $1 \leq i, j \leq d$ est $\Gamma_{j}{ }^{*} \Gamma_{i}$ où $\Gamma_{1}, \ldots, \Gamma_{d}$ sont les vecteurs colonnes de $\Gamma$ ).

Sous l'hypothèse d'existence d'un moment d'ordre 4 pour les v.a. $\left(X_{i}\right)$, on peut améliorer les vitesses de convergence des résultats (1.5) et (1.6) en exploitant la méthode de "moyennisation" suivante (cf. [9]) : on 
choisit deux réels $\alpha$ et $\gamma$ tels que $\frac{1}{\beta}<\alpha<\gamma \leq 1$ et on pose :

$$
\begin{aligned}
& w_{n}=n^{-\frac{\alpha+\gamma}{2}} \exp \left(2 \sum_{i=1}^{n} k^{-\alpha}\right) \text { pour } n \geq 1 \text { et } w_{0}=1 ; \\
& \tilde{m}_{n}=\left(\sum_{i=1}^{n} w_{i}\right)^{-1} \sum_{i=1}^{n} w_{i} X_{i} .
\end{aligned}
$$

Autrement dit $\left(\widetilde{m}_{n}\right)$ est l'estimateur des moindres carrés pondérés par le poids $\left(w_{n}\right)$ de $m$; il minimise le contraste $m \longmapsto \sum_{i=1}^{n} w_{i}\left\|X_{i}-m\right\|^{2}$. Cet estimateur satisfait aux propriétés suivantes :

$$
\widetilde{m}_{n} \underset{n \rightarrow \infty}{\longrightarrow} m \text { p.s. et } n^{\alpha / 2}\left(\widetilde{m}_{n}-m\right) \underset{n \rightarrow \infty}{\stackrel{\mathfrak{L}}{\longrightarrow}} \mathfrak{N}_{d}(0, \Gamma),
$$

car d'une part le "poids" $\left(w_{n}\right)$ vérifie :

$$
n^{-\alpha}\left(\sum_{i=1}^{n} w_{i}\right)^{2} \sim\left(\sum_{i=1}^{n} w_{i}^{2}\right) \sim n^{\alpha} w_{n}^{2}
$$

et d'autre part la marche aléatoire $\left(M_{n}\right)$ avec :

$$
M_{n}=\sum_{i=1}^{n} w_{i}\left(X_{i}-m\right)=\left(\sum_{i=1}^{n} w_{i}\right)\left(\widetilde{m}_{n}-m\right)
$$

est telle que : $\mathbb{E}\left(M_{n}{ }^{*} M_{n}\right)=\left(\sum_{i=1}^{n} w_{i}^{2}\right) \Gamma$.

L'estimateur obtenu par "moyennisation" arithmétique de $\widetilde{m}_{n}: \bar{m}_{n}=n^{-1} \sum_{i=1}^{n} \widetilde{m}_{i}$ converge en loi avec la vitesse optimale $\sqrt{n}: \sqrt{n}\left(\bar{m}_{n}-m\right) \underset{n \rightarrow \infty}{\stackrel{\mathfrak{L}}{\longrightarrow}} \mathfrak{N}_{d}(0, \Gamma)$.

Les transcriptions des propriétés (1.1-1.4) pour l'estimateur à poids $\widetilde{m}_{n}$ sont les suivantes :

$$
\begin{gathered}
(1-\alpha) n^{-(1-\alpha)} \sum_{k=1}^{n} k^{-\alpha} \delta_{\sqrt{k^{\alpha}}\left(\widetilde{m}_{k}-m\right)}^{\longrightarrow} \underset{n \rightarrow \infty}{\longrightarrow} \mathfrak{N}_{d}(0, \Gamma) \quad \text { p.s. } ; \\
(1-\alpha) n^{-(1-\alpha)} \sum_{k=1}^{n}\left(\widetilde{m}_{k}-m\right)^{*}\left(\widetilde{m}_{k}-m\right) \underset{n \rightarrow \infty}{\longrightarrow} \Gamma \quad \text { p.s. } ; \\
\check{\Gamma}_{n}=(1-\alpha) n^{-(1-\alpha)} \sum_{k=1}^{n}\left(\widetilde{m}_{k}-\bar{m}_{n}\right)^{*}\left(\widetilde{m}_{k}-\bar{m}_{n}\right) \underset{n \rightarrow \infty}{\longrightarrow} \Gamma \text { p.s. } \\
\operatorname{tr}\left\{\Gamma^{-1} \check{\Gamma}_{n} \Gamma^{-1 / 2}\right\} \underset{n \rightarrow \infty}{\longrightarrow} d \text { p.s. }
\end{gathered}
$$

Celles des propriétés (1.5) et (1.6) sont :

$$
\left\{\sqrt{\frac{n^{(1-\alpha)}}{1-\alpha}}\left[\check{\Gamma}_{n}-\Gamma\right], \sqrt{n}\left(\bar{m}_{n}-m\right)\right\} \underset{n \rightarrow \infty}{\stackrel{\mathfrak{L}}{\longrightarrow}} \mathfrak{N}_{d \times d}\left(0, \frac{1}{2} \Sigma\right) \otimes \mathfrak{N}_{d}(0, \Gamma)
$$




$$
\left\{\sqrt{\frac{n^{(1-\alpha)}}{1-\alpha}}\left[\operatorname{tr}\left\{\Gamma^{-1 / 2} \check{\Gamma}_{n} \Gamma^{-1 / 2}\right\}-d\right], \sqrt{n}\left(\bar{m}_{n}-m\right)\right\} \underset{n \rightarrow \infty}{\stackrel{\mathfrak{L}}{\longrightarrow}} \mathfrak{N}\left(0, \sigma^{2}\right) \otimes \mathfrak{N}_{d}(0, \Gamma)
$$

avec $\sigma^{2}=\operatorname{tr}\left(\Gamma^{2}\right)$.

L'exemple suivant présente l'avantage d'illustrer divers cadres considérés dans cet article et justifie la présentation des résultats en trois paragraphes.

Exemple 2. (Modèle autorégressif réel d'ordre p)

Soit $\left(\varepsilon_{n}\right)$ une suite de v.a. réelles, i.i.d. selon une loi centrée, de variance $\sigma^{2}>0$ et possédant un moment d'ordre 4 fini :

$$
\mathbb{E}\left(\varepsilon_{n}\right)=0, \quad \mathbb{E}\left(\varepsilon_{n}^{2}\right)=\sigma^{2} \text { et } \mathbb{E}\left(\varepsilon_{n}^{4}\right)<\infty,
$$

appelée bruit blanc.

Un processus autorégressif réel d'ordre $\mathbf{p}\left(\mathrm{AR}(\mathrm{p})\right.$ en abrégé), associé au bruit $\left(\varepsilon_{n}\right)$ s'écrit :

$$
Y_{n}=a_{1} Y_{n-1}+\cdots+a_{p} Y_{n-1}+\varepsilon_{n}, \quad n \geq 1
$$

où $a_{1}, \cdots, a_{p}$ sont des constantes réelles.

Pour un tel processus, on étudie l'estimation ponctuelle et ensembliste (régions de confiance) des paramètres $* \theta=\left(a_{1}, \ldots, a_{p}\right)$ et $\sigma^{2}$.

$$
\text { Notant : } \quad A=\left[\begin{array}{llllll}
a_{1} & \cdot & \cdot & \cdot & a_{p-1} & a_{p} \\
1 & 0 & \cdot & \cdot & \cdot & 0 \\
0 & \cdot & & & & \cdot \\
: & & \cdot & & & \vdots \\
0 & \cdot & \cdot & 0 & 1 & 0
\end{array}\right] \text {, }
$$

$\widetilde{\varepsilon}_{n}={ }^{*}\left(\varepsilon_{n}, 0, \cdots, 0\right)$ et $\widetilde{Y}_{n}$ le vecteur ${ }^{*}\left(Y_{n}, Y_{n+1}, \ldots, Y_{n+p-1}\right)$, on vérifie que :

$$
Y_{n}={ }^{*} \theta \widetilde{Y}_{n-1}+\varepsilon_{n}, \quad \widetilde{Y}_{n}=A \widetilde{Y}_{n-1}+\widetilde{\varepsilon}_{n}
$$

autrement dit $\left(\widetilde{Y}_{n}\right)$ est un processus autorégressif $p$-dimensionnel d'ordre $\mathbf{1}\left(A R_{p}(1)\right)$.

Le processus $\mathrm{AR}(\mathrm{p})$ est dit stable, purement instable ou purement explosif selon que tous les zéros de son polynôme caractéristique $P(z)=1-a_{1} z-\cdots-a_{p} z^{p}$ sont à l'extérieur strict, à l'intérieur strict ou sur le cercle unité.

Lorsque $P$ n'a pas de zéro de module égal à 1 , on dira ici que le processus $\mathrm{AR}(\mathrm{p})$ est mixte.

L'estimateur des moindres carrés ordinaires $\left(\widehat{\theta}_{n}\right)$ ou l'estimateur des moindres carrés pondérés $\left(\widetilde{\theta}_{n}\right)$ de $\theta$ peuvent-être définis par l'algorithme :

$$
\theta_{n+1}^{w}=\theta_{n}^{w}+w_{n}\left(P_{n}^{w}\right)^{-1} \widetilde{Y}_{n}\left(Y_{n+1}-{ }^{*} \theta_{n}^{w} \widetilde{Y}_{n}\right)
$$

avec :

$$
\begin{aligned}
& P_{n}=P_{n}^{w}=I_{p}+\sum_{k=0}^{n} w_{k} \widetilde{Y}_{k}^{*} \widetilde{Y}_{k} ; \theta_{0}^{w} \text { quelconque mais indépendant de }\left(Y_{n}, n \geq 1\right) ; \\
& \widehat{\theta}_{n}=\theta_{n}^{w} \text { si } w \equiv 1 ; \widetilde{\theta}_{n}=\theta_{n}^{w} \text { si }\left(w_{n}\right) \text { est le poids défini par }(1.7) .
\end{aligned}
$$

Pour de tels estimateurs, la relation :

$$
P_{n-1}^{w}\left(\theta_{n}^{w}-\theta\right)=\sum_{k=1}^{n} w_{k-1} \tilde{Y}_{k-1} \varepsilon_{k}
$$


joue un rôle fondamental dans l'étude asymptotique des erreurs d'estimation $\left(\theta_{n}^{w}-\theta\right)$ ou des erreurs de prédiction $\left(\Pi_{n}={ }^{*}\left(\theta_{n}^{w}-\theta\right) \widetilde{Y}_{n}\right)$ du fait que $M_{n}=\sum_{k=1}^{n} w_{k-1} \widetilde{Y}_{k-1} \quad \varepsilon_{k}$ est une martingale localement de carré intégrable au sens précisé ci-dessous en 1.3 .

Le comportement asymptotique des erreurs de prédiction est particulièrement utile pour l'étude de l'estimateur empirique de la covariance du bruit :

$$
\widehat{\sigma}_{n}^{2}=n^{-1} \sum_{i=1}^{n}\left(Y_{i}-{ }^{*} \widehat{\theta}_{i-1} \widetilde{Y}_{i-1}\right)^{2} \text { ou } \widetilde{\sigma}_{n}^{2}=n^{-1} \sum_{i=1}^{n}\left(Y_{i}-{ }^{*} \widetilde{\theta}_{i-1} \widetilde{Y}_{i-1}\right)^{2} .
$$

La propriété 1.4 s'étend à un modèle $\mathrm{AR}(\mathrm{p})$ stable, purement explosif ou mixte. En effet, on montrera ( $c f$. Sect. 2.2) que, dans le cas stable, la LFQ sur les erreurs d'estimation $\left(\widetilde{\theta}_{n}-\theta\right)$ s'écrit :

$$
(1-\alpha) n^{-(1-\alpha)} \sum_{k=1}^{n} *\left(\widetilde{\theta}_{k}-\theta\right) v_{n}^{-2} Q_{n}\left(\widetilde{\theta}_{k}-\theta\right) \underset{n \rightarrow \infty}{\longrightarrow} p \sigma^{2} \text { p.s. }
$$

où $Q_{n}=I_{p}+\sum_{k=0}^{n} w_{k}^{2} \widetilde{Y}_{k} * \widetilde{Y}_{k}, v_{n}^{2}=\sum_{k=1}^{n} w_{k}^{2}$.

Dans le cas purement explosif, la LFQ pour les erreurs d'estimation $\left(\widehat{\theta}_{n}-\theta\right)$ s'écrit :

$$
n^{-1} \sum_{k=1}^{n} *\left(\widehat{\theta}_{k}-\theta\right) Q_{k-1}\left(\widehat{\theta}_{k}-\theta\right) \underset{n \rightarrow \infty}{\longrightarrow} p \sigma^{2} \text { p.s. }
$$

où $Q_{n}=I_{p}+\sum_{k=0}^{n} \widetilde{Y}_{k} * \widetilde{Y}_{k}$ (cf. Sect. 3.2).

Les vitesses de convergence liées à ces deux propriétés seront également précisées.

La différence entre les deux formulations (1.18) et (1.19) de la LFQ s'explique par le fait que l'excitation $\left(Q_{n}\right)$ croit modérément dans le cas stable de l'exemple 2 et de manière exponentielle dans le cas purement explosif de celui-ci. Plus précisément : $\left(V_{n}^{-1} Q_{n}{ }^{*} V_{n}^{-1}\right)$ est une suite p.s. convergente avec $V_{n}=\sqrt{n^{\alpha}} w_{n} I_{p}$ et $V_{n+1}^{-1} V_{n} \underset{n \rightarrow \infty}{\longrightarrow} I_{p}$ dans le premier cas; et $V_{n}=A^{n}$ dans le second.

Dans le cadre de l'AR(p) mixte, ces deux situations sont simultanément présentes.

\subsection{Commentaires bibliographiques}

Pour des travaux antérieurs sur la loi forte quadratique des grands nombres LFQ relatifs aux martingales unidimensionnelles à temps discret ou continu, on pourra consulter $[3,4,9,19,21,24] \ldots$ Dans [22], une loi LFQ a été établie pour des algorithmes stochastiques vectoriels.

Les vitesses de convergence pour les processus empiriques logarithmiques unidimensionnels :

$$
H_{n}(x)=(\log n)^{-1} \sum_{k=1}^{n} \mathbf{1}_{\left\{\frac{S_{k}}{\sqrt{k}} \leq x\right\}} \text { où } S_{n}=\sum_{k=1}^{n} X_{k},
$$

ont été données par Csörgö et Horvath [11] via une approximation forte de la marche $\left(S_{n}\right)$ par une trajectoire brownienne. Dans cette perspective, des théorèmes limites avec poids ont été dégagés par le premier auteur dans [7] pour les martingales unidimensionnelles discrètes. Et par le second auteur dans [20] pour les martingales fonctionnelles additives d'un processus de Markov (à temps discret ou continu) récurrent. Berkes et al. [3] utilisent également cette approche pour généraliser les résultats de [11] à une loi appartenant au domaine d'attraction d'une loi stable symétrique d'indice $\alpha \in] 0,2[$. 
Les principaux résultats et leurs transcriptions statistiques sont présentés aux paragraphes 2,3 et 4 . Les preuves des résultats probabilistes sont donnés au paragraphe 5. Les illustrations relatives aux modèles autorégressifs sont clairement liées à ces résultats ; nous en ommettons les preuves par soucis de ne pas reproduire les mêmes schémas de démonstrations.

\subsection{Notations et hypothèses clés}

\section{Notations}

On note $\left(e_{1}, \ldots, e_{d}\right),\|$.$\| la base canonique et la norme euclidienne de \mathbb{R}^{d}$. Pour une matrice réelle carrée $A$ : ${ }^{*} A, \operatorname{tr}(A)$, Dét $A$ sont respectivement la matrice transposée, la trace, le déterminant. Si de plus $A$ est à spectre réel, $\lambda_{\max }(A)$ désignera sa plus grande valeur propre. La norme de $A$ est définie par $\|A\|^{2}=\lambda_{\max }\left(A^{*} A\right)$. Si $A_{1}$ et $A_{2}$ sont deux matrices carrées, on désigne par $\operatorname{Diag}\left(A_{1}, A_{2}\right)$ la matrice $\left[\begin{array}{cc}A_{1} & 0 \\ 0 & A_{2}\end{array}\right]$.

Les résultats porteront sur des suites de v.a. $M=\left(M_{n}\right)_{n \in \mathbb{N}}$ définies sur un espace de probabilité $(\Omega, \mathfrak{F}, \mathbb{F}, \mathbb{P})$ muni d'une filtration discrète $\mathbb{F}=\left(\mathfrak{F}_{n}\right)_{n \in \mathbb{N}}$, à valeurs dans $\mathbb{R}^{d}, \mathbb{F}$-adaptées et vérifiant pour tout $n$, les propriétés suivantes :

$$
M_{0}=0 ; \quad \mathbb{E}\left\{\Delta M_{n+1} / \mathfrak{F}_{n}\right\}=0 ; \quad \mathbb{E}\left\{\left\|\Delta M_{n+1}\right\|^{2} / \mathfrak{F}_{n}\right\}<\infty
$$

où

$$
\Delta M_{n+1}=M_{n+1}-M_{n} ; \quad n \in \mathbb{N} .
$$

Elles seront appelées des martingales localement de carré intégrable. Évidemment cela ne signifie pas nécessairement que les v.a. $\left(\left\|M_{n}\right\|\right)$ sont intégrables ou de carré intégrable. Pour de telles martingales, on note $[M]=\left([M]_{n}\right)_{n \in \mathbb{N}},\langle M\rangle=\left(\langle M\rangle_{n}\right)_{n \in \mathbb{N}}$ la variation quadratique et la variation quadratique prévisible définies respectivement par :

$[M]_{n}=\sum_{k=1}^{n}\left(\Delta M_{k}\right) *\left(\Delta M_{k}\right)$ et $[M]_{0}=0$,

$\Delta\langle M\rangle_{n+1}=\langle M\rangle_{n+1}-\langle M\rangle_{n}=\mathbb{E}\left\{\Delta M_{n+1}{ }^{*} \Delta M_{n+1} / \mathfrak{F}_{n}\right\}$ et $\langle M\rangle_{0}=0$.

\section{Hypothèses clés}

On donne $M=\left(M_{n}\right)_{n \in \mathbb{N}}$ une martingale satisfaisant (1.20) et $V=\left(V_{n}\right)_{n \in \mathbb{N}^{*}}$ une suite de matrices réelles $d \times d$, non aléatoires, inversibles, satisfaisant aux conditions de croissance régulière ou exponentielle ou mixte explicitées ci-dessous.

La propriété suivante est sous-jacente aux divers cadres de ce travail :

$$
Z_{n}=V_{n}^{-1} M_{n} \underset{n \rightarrow \infty}{\stackrel{\mathcal{L}}{\longrightarrow}} Z_{\infty}
$$

la limite $Z_{\infty}$ étant de la forme : $Z_{\infty}=\sum \stackrel{n \rightarrow \infty}{n}$ où $\eta$ est une v.a. (éventuellement dégénérée) et $\left(\sum(x)\right)$ est un processus spatial indépendant de $\eta$.

La propriété TLCG est en particulier vérifiée lorsque le couple (M,V) satisfait l'hypothèse H-2) ou bien les hypothèses H-1) et H'-2) suivantes (cf. commentaires ci-dessous).

- Hypothèse $\boldsymbol{H}$-1) sur le comportement asymptotique de la variation quadratique prévisible de $M$ : $C_{n}=V_{n}^{-1}\langle M\rangle_{n}{ }^{*} V_{n}^{-1} \underset{n \rightarrow \infty}{\longrightarrow} C$ p.s. où $C$ est une matrice aléatoire ou non.

- Hypothèse $\boldsymbol{H}$-2) sur le comportement asymptotique de la fonction caractéristique conditionnelle des v.a. $\left(V_{n}^{-1} \Delta M_{k}\right)$ :

Il existe une v.a. $\eta$ sur $(\Omega, \mathfrak{F}, \mathbb{P})$, éventuellement dégénérée, à valeurs dans un espace vectoriel de dimension finie 
$\mathfrak{X}$ et une probabilité $\mathcal{Q}$ sur l'espace $\mathfrak{C}=\mathfrak{C}\left(\mathfrak{X}, \mathbb{R}^{d}\right)$ des fonctions continues de $\mathfrak{X}$ dans $\mathbb{R}^{d}$, tels qu'en posant pour $u \in \mathbb{R}^{d}$ et $x \in \mathfrak{X}:$

$$
\Phi_{\infty}(x, u)=\int_{\mathbb{R}^{d}} \exp (i\langle u, \xi\rangle) \pi(x, d \xi), \Phi_{n}(u)=\prod_{k=1}^{n} \mathbb{E}\left\{\exp \left(i\left\langle u, V_{n}^{-1} \Delta M_{k}\right\rangle\right) / \mathfrak{F}_{k-1}\right\}
$$

$\left(\pi(x,\right.$.$) étant la loi image de \mathcal{Q}$ par l'application canonique $f \longmapsto f(x)$ de $\mathfrak{C}$ dans $\left.\mathbb{R}^{d}\right)$, on ait $\mathbb{P}$-presque-sûrement pour tout $u \in \mathbb{D}, \mathbb{D}$ dénombrable et dense dans $\mathbb{R}^{d}$ :

i) $\quad \Phi_{n}(u) \underset{n \rightarrow \infty}{\longrightarrow} \Phi_{\infty}(\eta, u)$, ii) $\Phi_{\infty}(\eta, u)$ non nulle.

- Condition de Lindeberg pour la convergence p.s. H'-2) :

$\forall \delta>0, \sum_{n=1}^{N} \mathbb{E}\left\{\left\|V_{N}^{-1} \Delta M_{n}\right\|^{2} \mathbf{1}_{\left\{\left\|V_{N}^{-1} \Delta M_{n}\right\|>\delta\right\}} / \mathfrak{F}_{n-1}\right\} \underset{N \rightarrow \infty}{\longrightarrow} 0$ p.s.

Le fait que les suites $\left(V_{n}^{-1}\langle M\rangle_{n}{ }^{*} V_{n}^{-1}\right)$ et $\left(V_{n}^{-1}[M]_{n}{ }^{*} V_{n}^{-1}\right)$ soient "proches" d'une certaine manière est important pour l'obtention d'une partie des résultats. Les deux hypothèses suivantes le traduisent :

- Hypothèse $\boldsymbol{H}$-3) sur le comportement asymptotique de la variation quadratique de $M$ :

$$
V_{n}^{-1}\left(\langle M\rangle_{n}-[M]_{n}\right){ }^{*} V_{n}^{-1} \underset{n \rightarrow \infty}{\longrightarrow} 0 \quad \text { p.s. }
$$

- Hypothèse $\boldsymbol{H}^{\prime}$-3) alternative à $\left.\mathrm{H}-3\right)$ :

La série $\sum\left[\log \left(\text { Dét } V_{n+1}\right)^{2}\right]^{-\beta} \mathbb{E}\left\{\left\|V_{n+1}^{-1} \Delta M_{n+1}\right\|^{2 \beta} / \mathfrak{F}_{n}\right\}$ est p.s. convergente pour un réel $\left.\left.\beta \in\right] 1,2\right]$.

Soit $\mu_{\infty}=\mu_{\infty}(\omega,$.$) la probabilité de transition (éventuellement non aléatoire), loi de la v.a. limite Z_{\infty}(\omega)=$ $\sum(\eta(\omega))$ dans le TLCG. La propriété suivante, automatiquement vérifiée sous H-1) et H'-2) (cf. commentaires ci-dessous) est naturelle :

- Hypothèse $\boldsymbol{H}$-4) sur le lien entre la covariance de $\mu_{\infty}$ et $C$ :

$$
C=\int x^{*} x d \mu_{\infty}(x) \text {. }
$$

\section{Remarques sur les hypothèses clés}

1) Sous l'hypothèse H-2) la convergence en loi dans le théorème de la limite centrale généralisé (TLCG) est stable, ce qui signifie que pour toute v.a. $d$-dimensionnelle $\varsigma$ sur $(\Omega, \mathfrak{F})$, le couple $\left(\varsigma, Z_{n}\right)$ converge en loi vers le couple $\left(\varsigma, Z_{\infty}\right)$. En particulier sous H-1) et H-2) :

$$
\left(Z_{n}, C_{n}\right) \underset{n \rightarrow \infty}{\stackrel{\mathcal{L}}{\longrightarrow}}\left(Z_{\infty}, C\right) \text {. }
$$

2) Les hypothèses H-1) et H'-2) impliquent que H-2) a lieu avec:

$$
\Phi_{\infty}(u)=\mathbb{E}\left\{\exp \left(-\frac{1}{2}{ }^{*} u C u\right)\right\}
$$

(cf. [6]). Et que l'on a : $V_{n}^{-1}[M]_{n}{ }^{*} V_{n}^{-1} \underset{n \rightarrow \infty}{\longrightarrow} C$ en probabilité (cf. [16]).

3) Des exemples éclairants ont été proposés dans [6] pour justifier la formulation assez technique de l'hypothèse H-2). Ces exemples montrent que la loi de la v.a. limite $Z_{\infty}$ dans TLCG n'est pas toujours un mélange de lois gaussiennes, même si la normalisation $\left(V_{n}\right)$ est à croissance régulière (i.e. vérifiant les conditions $(\mathcal{C})$ ci-dessous).

\section{RÉSUltats RELATIFs AUX MARTINGALES À CROISSANCE RÉGULIÈRE}

\section{1. Énoncés des théorèmes}

Les théorèmes de ce paragraphe s'appliquent à l'exemple 1 et au cas stable de l'exemple 2 .

- Conditions de croissance régulière $(\mathcal{C})$ sur la normalisation $\left(V_{n}\right)$

Il s'agit d'étendre la notion de croissance régulière d'une suite de réels à une normalisation matricielle. 
On dit que la suite de normalisation $\left(V_{n}\right)$ vérifie les conditions $(\mathcal{C})$, si les trois propriétés C-1), C-2), C-3) suivantes ont lieu où l'on note : $\Lambda_{n}=V_{n+1}^{-1} V_{n}$.

C-1) $V_{n}{ }^{*} V_{n} \leq V_{n+1}{ }^{*} V_{n+1}$ (au sens des matrices réelles symétriques positives);

C-2) si $n \rightarrow \infty, a_{n}=\operatorname{tr}\left(I-{ }^{*} \Lambda_{n} \Lambda_{n}\right)$ tend vers $0, A_{n}=\sum_{i=1}^{n} a_{i}$ croît vers $\infty$;

$$
a_{n}^{-1}\left(I-\Lambda_{n}{ }^{*} \Lambda_{n}\right) \underset{n \rightarrow \infty}{\longrightarrow} S \text { avec } S \text { inversible. }
$$

Les conditions $(\mathcal{C})$ sont notamment réalisées si :

$\Delta$ La normalisation est scalaire : $V_{n}=v_{n} I_{d}$ où $v_{n}=v(n)$ et $v: \mathbb{R}_{+} \longrightarrow \mathbb{R}_{+}$est une fonction continue, strictement croissante vers l'infini et vérifiant : $\frac{v_{n}}{v_{n+1}} \underset{n \rightarrow \infty}{\longrightarrow} 1$. Par exemple : $v(t)=t^{\rho}(\log t)^{\delta}$ avec $\rho>0$ et $\delta \in \mathbb{R}$ ou $\rho=0$ et $\delta>0$.

ム La normalisation est diagonale : $V_{n}=\operatorname{Diag}\left(v_{n}(1), \cdots, v_{n}(d)\right)$ où les $\left(v_{n}(j)\right)$ sont des normalisations scalaires du type précédent satisfaisant en outre la propriété suivante :

$$
\forall 1 \leq j \leq d, \quad a_{n}^{-1}\left[1-\left(\frac{v_{n}(j)}{v_{n+1}(j)}\right)^{2}\right] \underset{n \rightarrow \infty}{\longrightarrow} s_{j}^{2}, 0<s_{j}<\infty
$$

où $\left(a_{n}\right)$ est une suite telle que $\sum_{n} a_{n}=\infty$. Dans ce cas $S=\operatorname{Diag}\left\{s_{1}^{2}, \cdots, s_{d}^{2}\right\}$ et $\sum_{j=1}^{d} s_{j}^{2}=1$.

$\Delta$ Si les conditions $\mathrm{C}-1$ ) et C-2) sont vérifiées, alors on a C-3) dès que la propriété suivante est réalisée :

C-3-bis) $\quad \Lambda_{n}=I-a_{n} U+a_{n} \Delta_{n} ; \lim _{n \rightarrow \infty} \Delta_{n}=0$ pour une matrice $U$ telle que $S=U+{ }^{*} U$ soit définie positive.

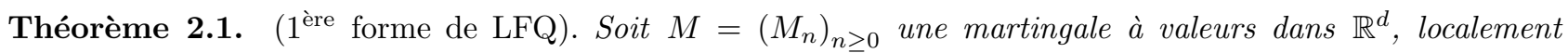
de carré intégrable (au sens de la Sect. 1.3) et $\left(V_{n}\right)$ une normalisation réalisant les conditions de croissance régulière $(\mathcal{C})$. On suppose que le couple $(M, V)$ satisfait aux hypothèses $\{H-1), H$-2), $\left[H\right.$-3) ou $\left.\left.H^{\prime}-3\right)\right]$ et $H$-4)\}.

1) Les propriétés suivantes sont alors vérifiées :

i) loi forte quadratique des grands nombres :

$$
\left(\log \left[\operatorname{Dét} V_{n}\right]^{2}\right)^{-1} \sum_{k=1}^{n}\left[1-\left(\frac{\text { Dét } V_{k}}{\text { Dét } V_{k+1}}\right)^{2}\right] V_{k}^{-1} M_{k}^{*} M_{k}^{*} V_{k}^{-1} \underset{n \rightarrow \infty}{\longrightarrow} C \text { p.s. ; }
$$

ii) loi du logarithme : $\left(\log \left[\operatorname{Dét} V_{n}\right]^{2}\right)^{-1 / 2} V_{n}^{-1} M_{n} \underset{n \rightarrow \infty}{\longrightarrow} 0$ p.s.

iii) $\lim _{n \rightarrow \infty}\left(\log \left[\operatorname{Dét} V_{n}\right]^{2}\right)^{-1} \sum_{k=1}^{n} * M_{k}\left(Q_{k}^{-1}-Q_{k+1}^{-1}\right) M_{k}=1$ p.s. sur $\{$ Dét $C>0\}$, avec $Q_{n}=I+\langle M\rangle_{n}$.

2) Ces propriétés sont en particulier vérifiées en substituant l'hypothèse $H^{\prime}$-2) au couple $\{H$-2), H-4)\}.

3) Lorsque les matrices $\left(V_{n}\right)$ sont diagonales, les propriétés i), ii) et iii) sont encore vérifiées sous les hypothèses $H$-1) et $\left.H^{\prime}-3\right)$.

Remarque 1. Sous l'hypothèse H'-3), la propriété ii) améliore le résultat suivant dû à Wei pour les suites régressives vectorielles $(c f .[10,13,14,26,27])$ :

$$
{ }^{*} M_{n} Q_{n}^{-1} M_{n}=O\left(\log \operatorname{Dét} Q_{n}\right) \text { p.s. sur }\left\{\log \operatorname{Dét} Q_{n} \rightarrow \infty\right\} .
$$

Corollaire 2.2. Dans le cadre du théorème 2.1, la loi forte logarithmique :

$$
\left(\log \left[\operatorname{Dét} V_{N}\right]^{2}\right)^{-1} \sum_{n=1}^{N}\left[1-\left(\frac{\text { Dét } V_{n}}{\operatorname{Dét} V_{n+1}}\right)^{2}\right] f\left(V_{n}^{-1} M_{n}\right) \underset{N \rightarrow \infty}{\longrightarrow} \int f d \mu_{\infty}
$$


a lieu hors d'un ensemble négligeable $\Delta$ indépendant de la fonction $f: \mathbb{R}^{d} \longrightarrow \mathbb{R}^{d}$, pourvu que celle-ci soit $\mu_{\infty}(\omega, \bullet)$-presque-partout continue pour $\omega \notin \Delta$ et que $x \longmapsto\|x\|^{-2}|f(x)|$ soit bornée à l'infini.

Pour préciser les vitesses de convergence correspondantes à la LFQ, on supposera dorénavant que la condition C-3-bis) est réalisée. Alors la propriété cruciale suivante, objet du lemme 5.2 a lieu sous l'hypothèse H-1) :

$$
\exp \left(-A_{N}\right) \sum_{n=1}^{N}\left[\exp \left(A_{n}\right)-\exp \left(A_{n-1}\right)\right] a_{n}^{-1} V_{n+1}^{-1}\left(\langle M\rangle_{n+1}-\langle M\rangle_{n}\right){ }^{*} V_{n+1}^{-1} \underset{N \rightarrow \infty}{\longrightarrow} \widetilde{C} \text { p.s. ; }
$$

$\widetilde{C}$ est la matrice aléatoire ou non définie par :

$$
\widetilde{C}=U C+C^{*} U ; \quad U \text { étant la matrice introduite dans C-3-bis). }
$$

Théorème 2.3 (TLC de la LFQ). Soit (M,V) un couple comme dans le théorème 2.1 avec une normalisation $V=\left(V_{n}\right)$ satisfaisant les conditions de croissance régulière $C$-1), $C$-2) et $C$-3-bis).

1) Pour toute matrice $R$ symétrique positive et de taille $d$ :

$$
\left\{A_{n}^{-1 / 2} \sum_{k=0}^{n} \operatorname{tr}\left\{\left(R-{ }^{*} \Lambda_{k} R \Lambda_{k}\right) V_{k}^{-1}\left(M_{k}{ }^{*} M_{k}-[M]_{k}\right){ }^{*} V_{k}^{-1}\right\}, V_{n}^{-1} M_{n}\right\} \underset{n \rightarrow \infty}{\stackrel{\mathcal{L}}{\longrightarrow}} \nu_{\infty} \otimes \mu_{\infty}
$$

où $\nu_{\infty}$ est la loi d'une v.a. de la forme $2 \sqrt{\operatorname{tr}\{\widetilde{C} R C R\}} G$, G étant une v.a. gaussienne centrée, réduite et indépendante de $C$.

2) Si la condition $C$-3-bis) a lieu avec $\Delta_{n}=O\left(A_{n}^{-3 / 2}\right) \quad(n \rightarrow \infty)$ et si pour un $\rho>\frac{1}{2}$ :

$$
A_{n}^{\rho}\left|\operatorname{tr}\left(V_{n}^{-1}[M]_{n}{ }^{*} V_{n}^{-1}\right)-\operatorname{tr}(C)\right|=O(1) \text { p.s. },(n \rightarrow \infty),
$$

ou bien

$$
A_{n}^{\rho} \mathbb{E}\left\{\left|\operatorname{tr}\left(V_{n}^{-1}[M]_{n}{ }^{*} V_{n}^{-1}\right)-\operatorname{tr}(C)\right|\right\}=O(1),(n \rightarrow \infty),
$$

alors prenant $R$ la solution de l'équation de Lyapounov :

$$
R U+{ }^{*} U R=I
$$

le résultat précédent s'écrit :

$$
\left\{\frac{1}{\sqrt{A_{n}}} \sum_{k=1}^{n} a_{k}\left\{\operatorname{tr}\left(V_{k}^{-1} M_{k}{ }^{*} M_{k}{ }^{*} V_{k}^{-1}\right)-\operatorname{tr}(C)\right\}, V_{n}^{-1} M_{n}\right\} \underset{n \rightarrow \infty}{\stackrel{\mathcal{L}}{\longrightarrow}} \nu_{\infty} \otimes \mu_{\infty} .
$$

Pour le résultat suivant il convient de renforcer légèrement l'hypothèse H'-3) de la manière suivante :

$\boldsymbol{H}$ "-3) La série $\sum\left[\log \left(\text { Dét } V_{n+1}\right)^{2}\right]^{-\beta / 2} \mathbb{E}\left\{\left\|V_{n+1}^{-1} \Delta M_{n+1}\right\|^{2 \beta} / \mathfrak{F}_{n}\right\}$ est p.s. convergente pour un réel $\beta \in$ ] $1,2]$.

Théorème 2.4 (LLI de la LFQ). Soit $M=\left(M_{n}\right)_{n \geq 0}$ une martingale à valeurs dans $\mathbb{R}^{d}$, localement de carré intégrable et $\left(V_{n}\right)$ une normalisation réalisant les conditions $\left.\left.\mathrm{C}-1\right), \mathrm{C}-2\right)$ et C-3-bis) avec $\Delta_{n}=o\left(A_{n}^{-1}\right)$. On

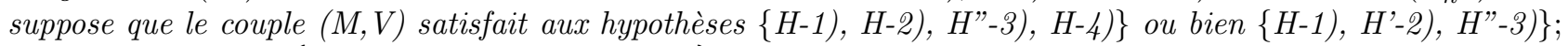
et que l'événement $\left\{\varlimsup_{n \rightarrow \infty} A_{n}^{-1 / 4}\left\|V_{n}^{-1} M_{n}\right\|=\infty\right\}$ est négligeable. On suppose en plus réalisée l'une des trois hypothèses suivantes :

\ la série $\sum_{n \geq 0} A_{n+1}^{-1 / 2}\left[\mathbb{E}\left\{\left\|R^{1 / 2} V_{n+1}^{-1} \Delta M_{n+1}\right\|^{2} / \mathfrak{F}_{n}\right\}-a_{n} \operatorname{tr}\{C\}\right]$ est p.s. convergente;

$R$ étant la matrice définie ci-dessus par (2.3) ;

$\Delta A_{n}^{\rho}\left|\operatorname{tr}\left(V_{n}^{-1}\langle M\rangle_{n}{ }^{*} V_{n}^{-1}\right)-\operatorname{tr}(C)\right|=O(1)$ p.s. ; 
ou

$\Delta A_{n}^{\rho} \mathbb{E}\left\{\left|\operatorname{tr}\left(V_{n}^{-1}\langle M\rangle_{n}{ }^{*} V_{n}^{-1}\right)-\operatorname{tr}(C)\right|\right\}=O(1)$, pour un $\rho>\frac{1}{2}(n \rightarrow \infty)$.

Alors le résultat de la partie 2) du théorème précédent est encore vrai. On a aussi une loi du logarithme itéré, à savoir :

$$
\varlimsup_{n \rightarrow \infty}\left(2 A_{n} \log \log A_{n}\right)^{-1 / 2}\left|\sum_{k=1}^{n} a_{k}\left(\left\|V_{k}^{-1} M_{k}\right\|^{2}-\operatorname{tr}\{C\}\right)\right|=2 \sqrt{\operatorname{tr}\{\widetilde{C} R C R\}} \text { p.s. }
$$

Théorème 2.5 (TLC et LLI de la LFQ avec normalisation scalaire). Soit $M=\left(M_{n}\right)_{n \geq 0}$ une martingale à valeurs dans $\mathbb{R}^{d}$, localement de carré intégrable. Et soit $v=\left(v_{n}\right)_{n>0}$ une normalisation scalaire telle que $v_{n} \underset{n \rightarrow \infty}{\longrightarrow} \infty$ en croissant, $\frac{v_{n}}{v_{n+1}} \underset{n \rightarrow \infty}{\longrightarrow} 1$.

1) Si $M$ satisfait aux hypothèses $\{H-1), H$-2), $[H-3)$ ou $\left.\left.\left.\left.H^{\prime}-3\right)\right], H-4\right)\right\}$ avec la normalisation $v\left(\right.$ ie $\left.V_{n}=v_{n} I_{d}\right)$, alors :

$$
\left\{\left(\log v_{n}^{2}\right)^{-1 / 2} \sum_{k=0}^{n}\left\{1-\left(\frac{v_{k}}{v_{k+1}}\right)^{2}\right\} v_{k}^{-2}\left(M_{k}{ }^{*} M_{k}-[M]_{k}\right), \frac{M_{n}}{v_{n}}\right\} \underset{n \rightarrow \infty}{\stackrel{\mathcal{L}}{\longrightarrow}} \nu_{\infty} \otimes \mu_{\infty}
$$

où, conditionnellement à $C=\Gamma, \nu_{\infty}$ est la loi d'une v.a. $\mathfrak{N}_{d \times d}(0, \mathfrak{B})$ indépendante de $C$ et $\mathfrak{B}=2 \Gamma \otimes \Gamma+$ $2^{\perp}(\operatorname{Vect}(\Gamma) * \operatorname{Vect}(\Gamma))$.

2) Supposons que les hypothèses $\{H-1), H-2), H$ "-3), H-4)\} sont satisfaites par le couple (M,v) et que l'une des trois hypothèses suivantes est réalisée :

\ la série $\sum_{n \geq 0}\left(\log v_{n+1}^{2}\right)^{-1 / 2} v_{n+1}^{-2}\left(\mathbb{E}\left\{\Delta M_{n+1}{ }^{*} \Delta M_{n+1} / \mathfrak{F}_{n}\right\}-\left(v_{n+1}^{2}-v_{n}^{2}\right) C\right)$

est p.s. convergente;

$\mathbf{\Delta} C_{n}=v_{n}^{-2}\langle M\rangle_{n}=C+O\left(\left(\log v_{n}^{2}\right)^{{ }^{\rho}}\right)$ p.s. ;

ou

$\Delta \mathbb{E}\left(\left\|C_{n}-C\right\|\right)=O\left(\left(\log v_{n}^{2}\right)^{-{ }^{\rho}}\right)$, pour un $\rho>\frac{1}{2}$.

a) Le résultat précédent est encore vrai en y remplaçant la suite $\left(v_{n}^{-2}[M]_{n}\right)$ par la matrice $C$.

b) Si l'événement $\Delta=\left\{\varlimsup_{n \rightarrow \infty}\left(\log v_{n}^{2}\right)^{-1 / 4} v_{n}^{-1}\left\|M_{n}\right\|=\infty\right\}$ est négligeable, alors pour tous vecteurs $x, y$ de la base cannonique de $\mathbb{R}^{d}$, on a :

$$
\begin{aligned}
& \varlimsup_{n \rightarrow \infty}\left(2 \log v_{n}^{2} \log \log \log v_{n}^{2}\right)^{-1 / 2}\left|\sum_{k=1}^{n}\left\{1-\left(\frac{v_{k}}{v_{k+1}}\right)^{2}\right\}\left[\frac{\left\langle x, M_{k}\right\rangle\left\langle y, M_{k}\right\rangle}{v_{k}^{2}}-{ }^{*} x C y\right]\right| \\
& =\sqrt{2\left({ }^{*} x C x\right)^{2}+2{ }^{*} x C x{ }^{*} y C y} \text { p.s. }
\end{aligned}
$$

c) Les propriétés données en a) et b) sont également vraies en rempaçant le couple d'hypothèses $\{H$-2), H-4)\} par H'-2). Elles ont aussi lieu sous l'hypothèse $H$-1) et la suivante :

$$
\left.\left.\sum_{n \geq 0} \mathbb{E}\left\{\left\|v_{n+1}^{-1} \Delta M_{n+1}\right\|^{2 \beta} / \mathfrak{F}_{n}\right\}<\infty \text { p.s. pour un réel } \beta \in\right] 1,2\right] \text {. }
$$

Et dans ce cas $\Delta$ est négligeable.

\subsection{Application à un $\mathbf{A R}(\mathbf{p})$ stable}

Dans le cadre de l'exemple 2, on peut énoncer les résultats suivants comme application des théorèmes du paragraphe 2.1. Ces résultats sont des cas particuliers de ceux établis dans [9] pour un modèle de régression stable ; ils ne seront donc pas prouvés ici.

Proposition 2.6. Soit $Y_{n+1}=a_{1} Y_{n}+\ldots+a_{p} Y_{n-p+1}+\varepsilon_{n+1}={ }^{*} \theta \widetilde{Y}_{n}+\varepsilon_{n+1}$ un processus AR $(p)$ (comme dans l'exemple 2) associé à un bruit blanc $\left(\varepsilon_{n}\right)$ ayant un moment d'ordre 4 fini et dont l'état initial $\widetilde{Y}_{0}$ est tel 
que $\mathbb{E}\left(\left\|\widetilde{Y}_{0}\right\|^{4}\right)<\infty$. On note $\widetilde{\theta}_{n}$ l'estimateur des moindres carrés pondérés de $\theta$, correspondant au poids $w_{n}$ défini par (1.7) ; et $\widetilde{\sigma}_{n}^{2}$ la variance empirique du bruit qui lui est associée.

Dans le cas stable, les estimateurs $\widetilde{\theta}_{n}, \bar{\theta}_{n}=n^{-1} \sum_{k=1}^{n} \widetilde{\theta}_{k}$ et $\tilde{\sigma}_{n}^{2}$ vérifient les propriétés suivantes :

i) Consistance forte de $\widetilde{\theta}_{n}, \bar{\theta}_{n}$ et $\widetilde{\sigma}_{n}^{2}$ :

$$
\begin{aligned}
& \left\|\widetilde{\theta}_{n}-\theta\right\|=O\left(\sqrt{\frac{\log \log n}{n^{\alpha}}}\right),\left\|\bar{\theta}_{n}-\theta\right\|=O\left(\sqrt{\frac{\log \log n}{n}}\right) \\
& \text { et }\left\|\widetilde{\sigma}_{n}^{2}-\sigma^{2}\right\|=o\left(n^{-(1-\alpha) / 2}\right) \text { p.s. } \\
& \text { ( } \left.\alpha \text { étant le paramètre introduit dans la définition du poids } w_{n}\right) \text {. }
\end{aligned}
$$

ii) Normalité asymptotique de $\widetilde{\theta}_{n}$ et de $\bar{\theta}_{n}$ :

$$
\sqrt{n^{\alpha}}\left(\widetilde{\theta}_{n}-\theta\right) \underset{n \rightarrow \infty}{\stackrel{\mathfrak{L}}{\longrightarrow}} \mathfrak{N}_{p}\left(0, T^{-1}\right), \sqrt{n}\left(\bar{\theta}_{n}-\theta\right) \underset{n \rightarrow \infty}{\stackrel{\mathfrak{L}}{\longrightarrow}} \mathfrak{N}_{p}\left(0, T^{-1}\right)
$$

où $T$ est la matrice définie par $T=\sum_{k=1}^{\infty} A^{k} e_{1}{ }^{*} e_{1}{ }^{*} A^{k}=\sigma^{-2} \lim _{n \rightarrow \infty} n^{-1} \sum_{k=1}^{n} \widetilde{Y}_{k}{ }^{*} \widetilde{Y}_{k}$ p.s.

iii) Lois fortes quadratiques sur les erreurs d'estimation de $\theta$ :

$$
\begin{gathered}
(1-\alpha) n^{-(1-\alpha)} \sum_{k=1}^{n}\left(\widetilde{\theta}_{k}-\theta\right) T^{*}\left(\widetilde{\theta}_{k}-\theta\right) \underset{n \rightarrow \infty}{\longrightarrow} I_{p} \text { p.s. } \\
(1-\alpha) n^{-(1-\alpha)} \sum_{k=1}^{n} *\left(\widetilde{\theta}_{k}-\theta\right)\left(v_{n}^{-2} Q_{n}\right)\left(\widetilde{\theta}_{k}-\theta\right) \underset{n \rightarrow \infty}{\longrightarrow} p \sigma^{2} \quad p . s .
\end{gathered}
$$

où $Q_{n}=I_{p}+\sum_{k=0}^{n} w_{k} \widetilde{Y}_{k} * \widetilde{Y}_{k}$ et $v_{n}=\sum_{k=1}^{n} w_{k}^{2}$. En conséquence, posant :

on $a$ :

$$
\check{\sigma}_{n}^{2}=\frac{1-\alpha}{p} n^{-(1-\alpha)} \sum_{k=1}^{n} *\left(\widetilde{\theta}_{k}-\bar{\theta}_{n}\right)\left(v_{n}^{-2} Q_{n}\right)\left(\widetilde{\theta}_{k}-\bar{\theta}_{n}\right)
$$

$$
\check{\sigma}_{n}^{2} \underset{n \rightarrow \infty}{\longrightarrow} \sigma^{2} \quad \text { p.s. }
$$

iv) Loi forte quadratique sur les erreurs de prédiction :

$$
\left(\frac{1-\alpha}{2}\right) n^{-(1-\alpha)} \sum_{k=1}^{n} \Pi_{k}^{2} \underset{n \rightarrow \infty}{\longrightarrow} p \sigma^{2} \quad \text { p.s. }
$$

avec $\Pi_{n}=*\left(\tilde{\theta}_{n}-\theta\right) \tilde{Y}_{n}$.

v) Normalité asymptotique du couple $\left(\check{\sigma}_{n}^{2}, \bar{\theta}_{n}\right)$ :

$$
\left\{\sqrt{\frac{p}{1-\alpha} n^{(1-\alpha)}}\left(\sigma^{-2} \check{\sigma}_{n}^{2}-1\right), \sqrt{n}\left(\bar{\theta}_{n}-\theta\right)\right\} \underset{n \rightarrow \infty}{\stackrel{\mathfrak{L}}{\longrightarrow}} \mathfrak{N}(0,1) \otimes \mathfrak{N}_{p}\left(0, T^{-1}\right) .
$$

vi) Vitesse de convergence presque-sûre de $\check{\sigma}_{n}^{2}$ :

$$
\varlimsup_{n \rightarrow \infty} \sqrt{\frac{p}{2(1-\alpha)} \frac{n^{(1-\alpha)}}{\log \log n}}\left|\sigma^{-2} \check{\sigma}_{n}^{2}-1\right|=1 \text { p.s. }
$$

\section{RÉSUltats RElatifs AUX MARTINGALES À CROISSANCE EXPONENTIELle}

\section{1. Énoncés des théorèmes}

Les résultats de ce paragraphe s'appliquent aux processus $\mathrm{AR}_{d}(\mathrm{p})$ purement explosifs et aux processus de branchement multitype $(c f .[21])$. 
- Conditions de croissance exponentielle $\left(\mathcal{C}^{\prime}\right)$ sur la normalisation $\left(V_{n}\right)$ :

La notion de croissance exponentielle s'étend de la manière suivante à une normalisation matricielle.

On dit que la suite de normalisation $\left(V_{n}\right)$ vérifie les conditions $\left(\mathcal{C}^{\prime}\right)$, si les trois propriétés C-1), C'-2), C'-3) suivantes ont lieu :

C'-2) $\quad \Lambda_{n} \underset{n \rightarrow \infty}{\longrightarrow} \Lambda_{\infty}$;

C'-3) $\quad \lambda_{\max }\left(\Lambda_{\infty}{ }^{*} \Lambda_{\infty}\right)<1$.

La condition C-1) a été introduite en 2.1.

Les conditions $\left(\mathcal{C}^{\prime}\right)$ sont en particulier vérifiées lorsque :

\ $V_{n}=\rho^{n} P$ avec $\rho>1$ et $P$ matrice régulière donnée (cas du processus de branchement multitype).

$\Delta V_{n}=A^{n}$ où $A$ est une matrice dont les valeurs propres sont de modules strictement supérieurs à 1 (cas de l'AR(p) purement explosif de l'exemple 2).

Théorème 3.1. ( $2^{\mathrm{e}}$ forme de LFQ). Soit $M=\left(M_{n}\right)_{n \geq 0}$ une martingale à valeurs dans $\mathbb{R}^{d}$, localement de carré intégrable et $\left(V_{n}\right)$ une normalisation satisfaisant aux conditions de croissance exponentielle $\left(\mathcal{C}^{\prime}\right)$. On suppose que le couple $(M, V)$ satisfait aux hypothèses $\left.\{H-1), H-2), H^{\prime}-3\right)$ et $\left.\left.\left.H-4\right)\right)\right\}$. Alors on a les deux propriétés :

$$
\frac{1}{N} \sum_{n=1}^{N} V_{n}^{-1} M_{n}^{*} M_{n}^{*} V_{n}^{-1} \underset{N \rightarrow \infty}{\longrightarrow} C \text { p.s. }
$$

et

$$
\frac{1}{N} \sum_{n=1}^{N}{ }^{*} M_{n}\left(Q_{n}^{-1}-Q_{n+1}^{-1}\right) M_{n} \underset{N \rightarrow \infty}{\longrightarrow} \operatorname{tr}\left(I-C^{-1 / 2} \Lambda_{\infty} C^{*} \Lambda_{\infty} C^{-1 / 2}\right) \text { p.s. }
$$

sur $\{$ Dét $C>0\}$, avec $Q_{n}=I+\langle M\rangle_{n}$.

En particulier, la première propriété implique que :

$$
\max _{1 \leq n \leq N}\left\|V_{n}^{-1} M_{n}\right\|=o(\sqrt{N}) \text { p.s. }
$$

Remarque 2. On peut énoncer un corollaire analogue au corollaire 2.2 (i.e. une loi forte logarithmique).

Théorème 3.2 ( $1^{\text {ère }}$ forme du TLC de la LFQ). Dans le cadre du théorème précédent, notant:

on a aussi les résultats suivants :

$$
D_{k}=V_{k}^{-1}\left(M_{k}{ }^{*} M_{k}-[M]_{k}\right){ }^{*} V_{k}^{-1},
$$

1) $\left\{\frac{1}{\sqrt{n}} \sum_{k=0}^{n}\left(D_{k}-\Lambda_{k} D_{k}{ }^{*} \Lambda_{k}\right), V_{n}^{-1} M_{n}\right\} \underset{n \rightarrow \infty}{\stackrel{\mathcal{L}}{\longrightarrow}}\left\{\sum^{\prime}(C)+{ }^{*} \sum^{\prime}(C), \sum(\eta)\right\}$

où $\sum^{\prime}(Y)$ est une v.a. gaussienne matricielle qu'on peut choisir indépendante du triplet $\left\{\eta, C,\left(\sum(x) ; x \in\right.\right.$ $\mathfrak{X})\}$ et dont la matrice de covariance est $\left(Y-\Lambda_{\infty} Y^{*} \Lambda_{\infty}\right) \otimes \Lambda_{\infty} Y^{*} \Lambda_{\infty}$ (Y étant une matrice symétrique positive).

2) Pour toute matrice $R$ 'symétrique, positive et de taille d, éventuellement aléatoire en tant que fonction mesurable du couple $(\eta, C)$ :

$$
\left\{\frac{1}{\sqrt{n}} \sum_{k=0}^{n} \operatorname{tr}\left\{\left(R^{\prime}-{ }^{*} \Lambda_{k} R^{\prime} \Lambda_{k}\right) V_{k}^{-1}\left(M_{k}{ }^{*} M_{k}-[M]_{k}\right){ }^{*} V_{k}^{-1}\right\}, V_{n}^{-1} M_{n}\right\} \underset{n \rightarrow \infty}{\stackrel{\mathcal{L}}{\longrightarrow}}\left\{\sigma(C) G, \sum(\eta)\right\}
$$

où $G$ est une v.a. gaussienne standard, indépendante du triplet $\left\{\eta, C,\left(\sum(x) ; x \in \mathfrak{X}\right)\right\}$ et $\sigma^{2}(C)=\sigma^{2}\left(C, R^{\prime}\right)$ $=4 \operatorname{tr}\left\{R^{\prime}\left(C-{ }^{*} \Lambda_{\infty} C \Lambda_{\infty}\right) R^{\prime} \Lambda_{\infty} C{ }^{*} \Lambda_{\infty}\right\}$. 
3) Si de plus, la suite $\left(n^{3 / 2}\left\|\Lambda_{n}-\Lambda_{\infty}\right\|\right)$ est bornée, alors pour $R^{\prime}=\sum_{k=0}^{\infty}{ }^{*} \Lambda_{\infty}^{k} \Lambda_{\infty}^{k}$ le résultat ci-dessus s'écrit :

$$
\left\{\frac{1}{\sqrt{n}} \sum_{k=0}^{n} \operatorname{tr}\left\{V_{k}^{-1}\left(M_{k}{ }^{*} M_{k}-[M]_{k}\right){ }^{*} V_{k}^{-1}\right\}, V_{n}^{-1} M_{n}\right\} \underset{n \rightarrow \infty}{\stackrel{\mathcal{L}}{\longrightarrow}}\left\{\sigma(C) . G, \sum(\eta)\right\} .
$$

Peut on substituer la suite $\left(V_{n}^{-1}\langle M\rangle_{n}{ }^{*} V_{n}^{-1}\right)$ à la suite $\left(V_{n}^{-1}[M]_{n}{ }^{*} V_{n}^{-1}\right)$ dans l'énoncé précédent ? L'étude de quelques exemples, notamment les processus de branchement multitype et les processus autorégressifs purement explosifs, montre que la méthodologie adoptée au paragraphe 2 ne s'applique pas ici. Le résultat suivant répond à la question posée. Il s'appuie sur les hypothèses supplémentaires suivantes :

H-5) : Les v.a. $X_{n}=V_{n}^{-1} \Delta M_{n}$ vérifient : $\left\|X_{n}\right\|=o\left(n^{1 / \delta}\right)$ p.s. avec $\delta \leq 4$.

H-6) : Les deux propriétés suivantes (où l'on note $\left\{e_{i}\right\}_{1 \leq i \leq d}$ la base canonique de $\mathbb{R}^{d}$ ) ont lieu :

i) $\mathcal{L}_{n}(i, j)=\frac{1}{n} \sum_{k=0}^{n} \mathbb{E}\left\{\left(X_{k+1}{ }^{*} X_{k+1}-\mathbb{E}\left[X_{k+1}{ }^{*} X_{k+1} / \mathfrak{F}_{k}\right]\right) e_{i}{ }^{*} e_{j}\left(X_{k+1}{ }^{*} X_{k+1}-\mathbb{E}\left[X_{k+1}{ }^{*} X_{k+1} / \mathfrak{F}_{k}\right]\right) / \mathfrak{F}_{k}\right\}$

$$
\underset{n \rightarrow \infty}{\longrightarrow} \mathcal{L}(i, j) \text { p.s., } \forall 1 \leq i, j \leq d
$$

ii) $\mathcal{J}_{n}(i, j)=\frac{1}{n} \sum_{k=0}^{n}\left(V_{k+1}^{-1} M_{k}\right) \mathbb{E}\left\{\left\langle X_{k+1}, e_{i}\right\rangle\left\langle X_{k+1}, e_{j}\right\rangle{ }^{*} X_{k+1} / \mathfrak{F}_{k}\right\} \underset{n \rightarrow \infty}{\longrightarrow} \mathcal{J}(i, j)$ p.s. ;

$\mathcal{L}(\mathrm{i}, \mathrm{j})$ et $\mathcal{J}(i, j)$ sont des v.a. matricielles.

Théorème 3.3. ( $2^{\mathrm{e}}$ forme du TLC de la LFQ). On se place dans le cadre du théorème 3.1 en ajoutant les hypothèses $H$-5) et $H-6)$.

1) Posant $\widetilde{D}_{k}=V_{k}^{-1}\left(M_{k}{ }^{*} M_{k}-\langle M\rangle_{k}\right){ }^{*} V_{k}^{-1}$, on $a$ :

$$
\left\{\frac{1}{\sqrt{n}} \sum_{k=0}^{n}\left(\widetilde{D}_{k}-\Lambda_{k} \widetilde{D}_{k}{ }^{*} \Lambda_{k}\right), V_{n}^{-1} M_{n}\right\} \underset{n \rightarrow \infty}{\stackrel{\mathcal{L}}{\longrightarrow}}\left\{\Sigma^{\prime}(C)+{ }^{*} \Sigma^{\prime}(C)+\Sigma^{\prime \prime}(C), \Sigma(\eta)\right\}
$$

où, conditionnellement à $C=Y,\left(\begin{array}{c}\sum^{\prime}(Y) \\ \sum^{\prime \prime}(Y)\end{array}\right)$ est une v.a. gaussienne matricielle qu'on peut choisir indépendante du triplet $\left\{\eta, C,\left(\sum(x) ; x \in \mathfrak{X}\right)\right\}$ et dont la structure de covariance $\triangle(Y)$ est donnée par la formule (3.2) ci-dessous.

2) Pour toute matrice $R$ 'symétrique positive et de taille $d$, éventuellement aléatoire en tant que fonction mesurable du couple $(\eta, C)$ :

$$
\left\{\frac{1}{\sqrt{n}} \sum_{k=0}^{n} \operatorname{tr}\left\{\left(R^{\prime}-{ }^{*} \Lambda_{k} R^{\prime} \Lambda_{k}\right) V_{k}^{-1}\left(M_{k}{ }^{*} M_{k}-\langle M\rangle_{k}\right){ }^{*} V_{k}^{-1}\right\}, V_{n}^{-1} M_{n}\right\} \underset{n \rightarrow \infty}{\stackrel{\mathcal{L}}{\longrightarrow}}\left\{\widetilde{\sigma}(C) G, \sum(\eta)\right\}
$$

où $G$ est une v.a. gaussienne standard, indépendante du triplet $\left\{\eta, C,\left(\sum(x) ; x \in \mathfrak{X}\right)\right\}$ et

$$
\begin{aligned}
\tilde{\sigma}^{2}(C) & =\tilde{\sigma}^{2}\left(C, R^{\prime}\right)=*\left(\begin{array}{c}
2 \operatorname{Vect}\left(R^{\prime}\right) \\
\operatorname{Vect}\left(R^{\prime}\right)
\end{array}\right) \triangle(C)\left(\begin{array}{c}
2 \operatorname{Vect}\left(R^{\prime}\right) \\
\operatorname{Vect}\left(R^{\prime}\right)
\end{array}\right) \\
& =4 \operatorname{tr}\left\{R^{\prime}\left(C-\Lambda_{\infty} C^{*} \Lambda_{\infty}\right) R^{\prime} C\right\}+\sum_{i=1}^{d} \sum_{j=1}^{d} * e_{i}\left(R^{\prime}\right)^{1 / 2}(\mathcal{L}(i, j)+4 \mathcal{J}(i, j))\left(R^{\prime}\right)^{1 / 2} e_{j}
\end{aligned}
$$


3) Si de plus la suite $\left(n\left(\Lambda_{n}-\Lambda_{\infty}\right)\right)$ est bornée, le résultat ci-dessus s'écrit sous la forme suivante que l'on obtient en prenant $R^{\prime}=\sum_{k=0}^{\infty}{ }^{*} \Lambda_{\infty}^{k} \Lambda_{\infty}^{k}$ :

$$
\left\{\frac{1}{\sqrt{n}} \sum_{k=0}^{n} \operatorname{tr}\left\{V_{k}^{-1}\left(M_{k}{ }^{*} M_{k}-\langle M\rangle_{k}\right){ }^{*} V_{k}^{-1}\right\}, V_{n}^{-1} M_{n}\right\} \underset{n \rightarrow \infty}{\stackrel{\mathcal{L}}{\longrightarrow}}\left\{\widetilde{\sigma}(C) G, \sum(\eta)\right\} .
$$

La matrice de covariance $\triangle(Y)$ est donnée par les formules suivantes :

$$
\left\{\begin{aligned}
\operatorname{cov}\left(\operatorname{Vect} \sum^{\prime}(Y), \operatorname{Vect} \sum^{\prime}(Y)\right) & =\left(Y-\Lambda_{\infty} Y^{*} \Lambda_{\infty}\right) \otimes \Lambda_{\infty} Y^{*} \Lambda_{\infty} \\
\operatorname{cov}\left(\operatorname{Vect} \sum^{\prime}(Y), \operatorname{Vect} \sum^{\prime \prime}(Y)\right) & =\mathcal{J} \\
\operatorname{cov}\left(\operatorname{Vect} \sum^{\prime \prime}(Y), \operatorname{Vect} \sum^{\prime \prime}(Y)\right) & =\mathcal{L}
\end{aligned}\right.
$$

Théorème 3.4. (LLI de la LFQ). On se place dans le cadre du théorème 3.3 en supposant que la suite $\left(n\left(\Lambda_{n}-\Lambda_{\infty}\right)\right)$ est bornée et en renforçant l'hypothèse $\left.H-1\right)$ de la manière suivante :

$\boldsymbol{\Delta} V_{n}^{-1}\langle M\rangle_{n}{ }^{*} V_{n}^{-1}=C+O\left(n^{-\rho}\right)$ p.s. pour un $\rho>1$; et $C$ est p.s. inversible.

1) La version suivante du résultat 3) du théorème 3.3 a lieu:

$$
\left\{\frac{1}{\sqrt{n}} \sum_{k=1}^{n}\left({ }^{*} M_{k} Q_{k}^{-1} M_{k}-\mathrm{d}\right), V_{n}^{-1} M_{n}\right\} \underset{n \rightarrow \infty}{\stackrel{\mathcal{L}}{\longrightarrow}}\left\{\widetilde{\sigma}(C) G, \sum(\eta)\right\},
$$

à condition de prendre $R^{\prime}=\sum_{k=0}^{\infty}{ }^{*} \Lambda_{\infty}^{k} C^{-1} \Lambda_{\infty}^{k}$ dans l'expression de $\widetilde{\sigma}(C)=\widetilde{\sigma}\left(C, R^{\prime}\right)$ défini par (3.1) et de poser $Q_{n}=I+\langle M\rangle_{n}$.

2) On a aussi une loi du logarithme itéré :

$$
\varlimsup_{n \rightarrow \infty}(2 n \log \log n)^{-1 / 2}\left|\sum_{k=1}^{n}\left({ }^{*} M_{k} Q_{k}^{-1} M_{k}-\mathrm{d}\right)\right|=\widetilde{\sigma}(C) p . s .
$$

à condition de substituer à l'hypothèse $\left.\mathrm{H}^{\prime}-3\right)$ la suivante :

$$
\left.\left.\mathbf{H} "{ }^{\prime-3)} \sum_{k \geq 1} k^{-\beta} \mathbb{E}\left\{\left\|V_{k+1}^{-1} \Delta M_{k+1}\right\|^{4} / \mathfrak{F}_{k}\right\}<\infty \text { p.s. pour un } \beta \in\right] 1,2\right] .
$$

\subsection{Application à un $\mathbf{A R}(\mathbf{p})$ purement explosif}

Dans le cadre de l'exemple 2, on peut énoncer les résultats suivants comme application du théorème 3.4.

Proposition 3.5. Soit $Y_{n+1}=a_{1} Y_{n}+\ldots+a_{p} Y_{n-p+1}+\varepsilon_{n+1}={ }^{*} \theta \widetilde{Y}_{n}+\varepsilon_{n+1}$ un processus AR(p) satisfaisant aux hypothèses de la proposition 2.6. On suppose aussi que la fonction caractéristique commune des v.a. $\left(\varepsilon_{n}\right)$ a au plus un nombre dénombrable de zéros. Alors, dans le cas purement explosif, l'estimateur $\widehat{\theta}_{n}$ des moindres carrés ordinaires de $\theta$ vérifie les propriétés suivantes.

i) Consistance forte de $\widehat{\theta}_{n}$ :

$$
{ }^{*} A^{n}\left(\widehat{\theta}_{n}-\theta\right)=o\left(n^{1 / 4}\right) \text { p.s. }(n \rightarrow \infty) .
$$

ii) Convergence en loi de $\widehat{\theta}_{n}$ :

$$
{ }^{*} A^{n}\left(\widehat{\theta}_{n}-\theta\right) \underset{n \rightarrow \infty}{\stackrel{\mathfrak{L}}{\longrightarrow}} C^{-1} \Sigma(\eta)
$$


où : $\eta=\widetilde{Y}_{0}+\sum_{k=1}^{\infty} \varepsilon_{k} A^{-k} e_{1}=\lim _{n \rightarrow \infty} A^{-n} \widetilde{Y}_{n} \quad$ p.s. ; C est la matrice aléatoire p.s. inversible, définie par : $C=\sum_{k=1}^{\infty} A^{-k} \eta^{*} \eta^{*} A^{-k}=\lim _{n \rightarrow \infty} A^{-n} Q_{n-1}{ }^{*} A^{-n}$ p.s. ; et $\Sigma(x)=\sum_{k=1}^{\infty} \varepsilon_{k}^{\prime} A^{-k} x$ pour une suite de v.a. $\left(\varepsilon_{k}^{\prime} ; k \geq 1\right)$ i.i.d. selon la loi du bruit et indépendante de celui-ci.

iii) Lois fortes quadratiques sur les erreurs d'estimation de $\theta$ :

$$
n^{-1} \sum_{k=1}^{n} A^{k}\left(\widehat{\theta}_{k}-\theta\right) *\left(\widehat{\theta}_{k}-\theta\right) * A^{k} \underset{n \rightarrow \infty}{\longrightarrow} \sigma^{2} C^{-1} \text { p.s. }
$$

En conséquence :

$$
n^{-1} \mathfrak{T}_{n}=n^{-1} \sum_{k=1}^{n} *\left(\widehat{\theta}_{k}-\theta\right) Q_{k-1}\left(\widehat{\theta}_{k}-\theta\right) \underset{n \rightarrow \infty}{\longrightarrow} p \sigma^{2} \quad p . s .
$$

et

$$
{\stackrel{\vee}{v_{n}}}_{n}=(n p)^{-1} \sum_{k=1}^{n} *\left(\widehat{\theta}_{k}-\widehat{\theta}_{n}\right) Q_{k-1}\left(\widehat{\theta}_{k}-\widehat{\theta}_{n}\right) \underset{n \rightarrow \infty}{\longrightarrow} \sigma^{2} \quad p . s .
$$

iv) Loi forte quadratique sur les erreurs de prédiction :

$$
n^{-1} \sum_{k=1}^{n} \Pi_{k}^{2} \underset{n \rightarrow \infty}{\longrightarrow} \sigma^{2 *} \eta C^{-1} \eta \quad p . s .
$$

v) Normalité asymptotique de $\mathfrak{T}_{n}$ et $\stackrel{\vee}{\sigma}_{n}^{2}$ :

$$
\begin{gathered}
\left\{\frac{1}{\sqrt{n}}\left\{\mathfrak{T}_{n}-n p \sigma^{2}\right\},{ }^{*} A^{n}\left(\widehat{\theta}_{n}-\theta\right)\right\} \underset{n \rightarrow \infty}{\stackrel{\mathfrak{L}}{\longrightarrow}}\{\tau G, \Sigma(\eta)\} ; \\
\left\{p \sqrt{n}\left\{\widehat{\sigma}_{n}^{2}-\sigma^{2}\right\},{ }^{*} A^{n}\left(\widehat{\theta}_{n}-\theta\right)\right\} \underset{n \rightarrow \infty}{\stackrel{\mathfrak{L}}{\longrightarrow}}\{\tau G, \Sigma(\eta)\}
\end{gathered}
$$

où $G$ est une v.a. gaussienne standard, indépendante du couple $\left\{\eta,\left(\Sigma(x) ; x \in \mathbb{R}^{p}\right)\right\}$ et $\tau^{2}$ est la v.a. définie par:

$$
\tau^{2}=4 \sigma^{4} \operatorname{tr}\left\{R^{\prime}\left(C-A^{-1} C^{*} A^{-1}\right) R^{\prime} C\right\}+\operatorname{Var}\left(\varepsilon_{1}^{2}\right) \operatorname{tr}\left\{\left[R^{\prime}\left(C-A^{-1} C^{*} A^{-1}\right) R^{\prime} C\right]^{2}\right\}
$$

avec $R^{\prime}=\sum_{k=0}^{\infty} A^{-k} C^{-1 *} A^{-k}$

vi) Vitesse de convergence p.s. de $n^{-1} \mathfrak{T}_{n}$ et $\stackrel{\vee}{\sigma}_{n}^{2}$ :

$$
\varlimsup \sqrt{\frac{n}{2 \log \log n}}\left|n^{-1} \mathfrak{T}_{n}-p \sigma^{2}\right|=\tau, \varlimsup \sqrt{\frac{n}{2 \log \log n}}\left|\sigma_{n}^{2}-\sigma^{2}\right|=\frac{\tau}{p} \text { p.s. }
$$

Remarque. Dans le cadre de la proposition $3.5, \widehat{\sigma}_{n}^{2}\left(1-{ }^{*} \widetilde{Y}_{n} Q_{n}^{-1} \widetilde{Y}_{n}\right)$ est un estimateur fortement consistant de $\sigma^{2}(c f .[14,25])$. 


\section{RÉsultats Relatifs aux martingales À CROissance miXte}

\section{1. Énoncés des résultats}

Les résultats de ce paragraphe sont particulièrement adaptés à l'AR $\mathrm{A}_{d}(\mathrm{p})$ mixte.

Dans l'énoncé suivant, on envisage une normalisation $\left(V_{n}\right)$ de la forme :

$$
V_{n}=\operatorname{Diag}\left(V_{n}(1), V_{n}(2)\right)
$$

avec des matrices $\left(V_{n}(2)\right)$ de taille $d_{2} \times d_{2}$, satisfaisant aux conditions de croissance exponentielle $\left(\mathcal{C}^{\prime}\right)$ et des matrices $\left(V_{n}(1)\right)$ de taille $d_{1} \times d_{1}$, satisfaisant aux conditions de croissance régulière $(\mathcal{C})$. Plus précisément, on suppose que les suites de réels :

$$
a_{n}(1)=\operatorname{tr}\left(I-{ }^{*} \Lambda_{n}(1) \Lambda_{n}(1)\right) \text { et } A_{N}(1)=\sum_{n=1}^{N} a_{n}(1) \text { où } \Lambda_{n}(1)=V_{n+1}^{-1}(1) V_{n}(1)
$$

vérifient :

$$
\left.\left.n\left(1-\frac{a_{n+1}(1)}{a_{n}(1)}\right) \underset{n \rightarrow \infty}{\longrightarrow} \alpha, \frac{n a_{n}(1)}{A_{n}(1)} \underset{n \rightarrow \infty}{\longrightarrow} 1-\alpha(n \rightarrow \infty) \text { pour un } \alpha \in\right] 0,1\right]
$$

Théorème 4.1. ( $3^{\mathrm{e}}$ forme de LFQ). Soit $M=\left(M_{n}(1), M_{n}(2)\right)_{n \geq 0}$ une martingale à valeurs dans $\mathbb{R}^{d_{1}} \times$ $\mathbb{R}^{d_{2}}$, localement de carré intégrable. Et soit $V=\left(V_{n}\right)$ une normalisation satisfaisant aux conditions (4.1) et (4.2). On suppose que le couple $(M, V)$ vérifie les hypothèses $\{H-1)$, H-2) et $H$-4)\} et que les couples $(M(j), V(j)), j=1,2$, vérifient l'hypothèse $\left.H^{\prime}-3\right)$. Alors on a :

$$
A_{N}^{-1}(1) \sum_{n=1}^{N} a_{n}(1) V_{n}^{-1} M_{n}^{*} M_{n}^{*} V_{n}^{-1} \underset{n \rightarrow \infty}{\longrightarrow} C \quad \text { p.s. }
$$

et

$$
\frac{1}{N} \sum_{n=1}^{N}{ }^{*} M_{n}\left(Q_{n}^{-1}-Q_{n+1}^{-1}\right) M_{n} \underset{n \rightarrow \infty}{\longrightarrow} \operatorname{tr}\left(I-C(2)^{-1 / 2} \Lambda_{\infty}(2) C(2)^{*} \Lambda_{\infty}(2) C(2)^{-1 / 2}\right) \text { p.s. }
$$

sur $\{$ Dét $C>0\}$ où l'on note:

$$
\Lambda_{\infty}(2)=\lim _{n \rightarrow \infty} V_{n+1}^{-1}(2) V_{n}(2), C(2)=\lim _{n \rightarrow \infty} V_{n}^{-1}(2)\langle M\rangle_{n}(2){ }^{*} V_{n}(2) \text { et } Q_{n}=I+\langle M\rangle_{n} .
$$

Dans le second résultat de ce paragraphe, on précise les vitesses de convergence p.s. et en loi de la LFQ du théorème 4.1. Pour cela, on suppose d'une part que la normalisation mixte $\left(V_{n}\right)$ vérifie les conditions (4.3) et (4.4) ; et que d'autre part le couple $(M, V)$ vérifie les hypothèses (4.5-4.7).

(4.3) La normalisation à croissance régulière $\left(V_{n}(1)\right)$ est scalaire : $V_{n}(1)=v_{n} I_{d_{1}}$ où $\left(v_{n}\right)$ est une suite de réels positifs croissant vers l'infini et telle que :

$$
1-\left(\frac{v_{n}}{v_{n+1}}\right)^{2}=s^{2} n^{-\alpha}+o\left(n^{-(1+\alpha)}\right) \quad(n \rightarrow \infty)
$$

pour un $s>0$ et un $\alpha \in\left[\frac{3}{4}, 1[\right.$.

(4.4) La normalisation à croissance exponentielle $\left(V_{n}(2)\right)$ est telle que :

$$
\left\|\Lambda_{n}(2)-\Lambda_{\infty}(2)\right\|=O\left(n^{-\alpha / 2}\right) \quad(n \rightarrow \infty) .
$$


(4.5) Le couple $(M, V)$ vérifie la variante suivante de l'hypothèse $\mathrm{H}-1)$ :

$\Delta v_{n}^{-2}\langle M(1)\rangle_{n}=C(1)+O\left(\left(\log v_{n}^{2}\right)^{-\rho_{1}}\right)$ p.s., $\rho_{1}>\frac{1}{2}$ et $C(1)$ est non aléatoire et inversible.

\ $V_{n}^{-1}(2)\langle M(2)\rangle_{n}{ }^{*} V_{n}^{-1}(2)=C(2)+O\left(n^{-\rho_{2}}\right)$ p.s., $\rho_{2}>1$ et $C(2)$ est p.s. inversible.

(4.6) Le couple $\left\{\left(M_{n}(1)\right),\left(v_{n}\right)\right\}$ est tel que :

$$
\sum_{n \geq 0} \mathbb{E}\left\{\left\|v_{n+1}^{-1} \Delta M_{n+1}(1)\right\|^{2 \beta} / \mathfrak{F}_{n}\right\}<\infty \text { p.s. }
$$

pour un $\beta \in] 1,2]$.

(4.7) Le couple $\left\{\left(M_{n}(2)\right),\left(V_{n}(2)\right)\right\}$ vérifie les hypothèses $\left.\left.\{\mathrm{H}-2), \mathrm{H}-6\right)\right\}$ et l'hypothèse H-5) avec $\delta=$ $\left(\alpha-\frac{1}{2}\right)^{-1} \leq 4$.

Théorème 4.2. (TLC et LIL de la LFQ). Soit $(M, V)$ un couple comme dans le théorème 4.1, vérifiant les hypothèses (4.3-4.7). Alors on a les résultats suivants:

1) $V_{n}^{-1} Q_{n}{ }^{*} V_{n}^{-1}=C+o\left(n^{-(1-\alpha) / 2}\right)$ p.s. avec $C=\operatorname{Diag}\{C(1), C(2)\}$.

2) $\left\{\sqrt{\frac{1-\alpha}{n^{1-\alpha}}} \sum_{k=1}^{n} k^{-\alpha}\left({ }^{*} M_{k} Q_{k}^{-1} M_{k}-\mathrm{d}\right), v_{n}^{-1} M_{n}(1), V_{n}^{-1}(2) M_{n}(2)\right\} \underset{n \rightarrow \infty}{\stackrel{\mathcal{L}}{\longrightarrow}}\left\{\frac{2 \sqrt{d_{1}}}{s^{2}} G, C^{1 / 2}(1) N, \Sigma_{2}(\eta)\right\}$ où $(G, N)$ est un couple de v.a. gaussiennes de loi $\mathfrak{N}(0,1) \otimes \mathfrak{N}_{d_{1}}\left(0, I_{d_{1}}\right)$ et indépendant du triplet $\left\{\eta, C(2),\left(\Sigma_{2}(x) ; x \in \mathfrak{X}\right)\right\}$.

3) $\varlimsup_{n \rightarrow \infty}\left(\frac{2}{1-\alpha} n^{1-\alpha} \log \log n\right)^{-1 / 2}\left|\sum_{k=1}^{n} k^{-\alpha}\left({ }^{*} M_{k} Q_{k}^{-1} M_{k}-\mathrm{d}\right)\right|=\frac{2 \sqrt{d_{1}}}{s^{2}}$ p.s.

\subsection{Application à l'AR(p) mixte}

En guise d'application des deux théorèmes précédents, on peut énoncer la proposition suivante qui complète les deux propositions 2.6 et 3.5 .

Proposition 4.3. Soit $Y_{n+1}=a_{1} Y_{n}+\ldots+a_{p} Y_{n-p+1}+\varepsilon_{n+1}={ }^{*} \theta \widetilde{Y}_{n}+\varepsilon_{n+1}$ un processus AR(p) dont le bruit $\left(\varepsilon_{n}\right)$ et l'état initial $\widetilde{Y}_{0}$ vérifient les propriétés de la proposition 3.5. On désigne par $\widetilde{\theta}_{n}$ l'estimateur des moindres carrés pondérés de $\theta$, correspondant au poids $w_{n}=n^{-(\alpha+\gamma) / 2} \exp \left\{2 \sum_{k=1}^{n} k^{-\alpha}\right\}$ avec $\alpha \in\left[\frac{3}{4}, 1[\right.$ et $\alpha<\gamma \leq 1$ et par $\bar{\theta}_{n}$ son moyennisé : $\bar{\theta}_{n}=n^{-1} \sum_{k=1}^{n} \tilde{\theta}_{k}$.

Dans le cas mixte, $\widetilde{\theta}_{n}$ et $\bar{\theta}_{n}$ vérifient les propriétés suivantes où $\mathcal{G}=\left(\begin{array}{l}\mathcal{G}(1) \\ \mathcal{G}(2)\end{array}\right) \begin{aligned} & p_{1} \times p \\ & p_{2} \times p\end{aligned}$ est une matrice réelle, régulière, telle que la matrice compagne $\mathrm{A}$ de l'AR(p) $\left(Y_{n}\right)$ admette la décomposition : $\mathcal{G} \mathrm{A} \mathcal{G}^{-1}=$ $\operatorname{Diag}\{\mathrm{A}(1), \mathrm{A}(2)\} ; \mathrm{A}(1)[$ resp. $\mathrm{A}(2)]$ étant une matrice $p_{1} \times p_{1}\left[\right.$ resp. $\left.p_{2} \times p_{2}\right]$ dont le spectre est à l'intérieur [resp. à l'extérieur] strict du cercle unité.

i) Consistance forte de $\widetilde{\theta}_{n}$ et $\bar{\theta}_{n}$ :

ii) Convergence en loi de $\widetilde{\theta}_{n}$ :

$$
\left\|\widetilde{\theta}_{n}-\theta\right\|=O\left(\sqrt{\frac{\log \log n}{n^{\alpha}}}\right) \text { et }\left\|\bar{\theta}_{n}-\theta\right\|=O\left(\sqrt{\frac{\log \log n}{n}}\right) \text { p.s. }
$$

$$
\begin{gathered}
\mathcal{G}_{n}\left(\widetilde{\theta}_{n}-\theta\right) \underset{n \rightarrow \infty}{\stackrel{\mathfrak{L}}{\longrightarrow}} \mathfrak{N}_{p_{1}}\left(0, T(1)^{-1}\right) \otimes \nu \\
\text { où : } \\
\mathcal{G}_{n}=\operatorname{Diag}\left\{n^{\alpha / 2} I_{p_{1}},{ }^{*} \mathrm{~A}(2)^{n}\right\}{ }^{*} \mathcal{G}^{-1} ; T(1)=\sum_{k=1}^{\infty} A(1)^{k} f_{1}{ }^{*} f_{1}{ }^{*} A(1)^{k} \text { avec }^{*} f_{1}=(1,0, \ldots, 0)_{1 \times p_{1}} ;
\end{gathered}
$$


$\nu$ est la loi de la v.a. produit $T(2)^{-1} \Sigma_{2}(\eta)$ avec $\eta=\mathcal{G}(2) \widetilde{Y}_{0}+\sum_{k=1}^{\infty} \varepsilon_{k} \mathrm{~A}(2)^{-k} g_{1}$,

${ }^{*} g_{1}=(1,0, \ldots, 0)_{1 \times p_{2}}, \quad T(2)=\sum_{k=1}^{\infty} \mathrm{A}(2)^{-k} \eta^{*} \eta^{*} \mathrm{~A}(2)^{-k}, \Sigma_{2}(\eta)=\sum_{k=1}^{\infty} \varepsilon_{k}^{\prime} \mathrm{A}(2)^{-k} \eta, \quad\left(\varepsilon_{n}^{\prime}\right)$ étant un bruit blanc identique à $\left(\varepsilon_{n}\right)$ et indépendant de lui.

iii) Théorème de la limite centrale presque-sûre :

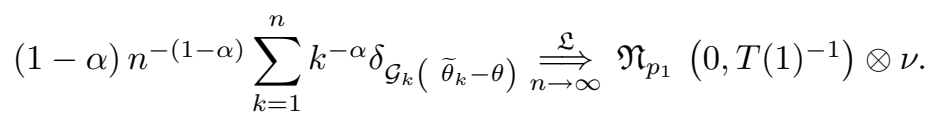

iv) Loi forte quadratique sur les erreurs d'estimation $\operatorname{de} \theta$ :

$(1-\alpha) n^{-(1-\alpha)} \mathfrak{T}_{n}=2(1-\alpha) n^{-(1-\alpha)} \sum_{k=1}^{n} k^{-\alpha} w_{k}^{-1 *}\left(\widetilde{\theta}_{k}-\theta\right) P_{k-1}\left(\widetilde{\theta}_{k}-\theta\right) \underset{n \rightarrow \infty}{\longrightarrow}\left(p+p_{2}\right) \sigma^{2} p . s$.

avec $P_{n}=I_{p}+\sum_{k=0}^{n} w_{k} \widetilde{Y}_{k}{ }^{*} \widetilde{Y}_{k}$. En conséquence :

$$
\mathfrak{S}_{n}=2(1-\alpha) n^{-(1-\alpha)} \sum_{k=1}^{n} k^{-\alpha} w_{k}^{-1} *\left(\widetilde{\theta}_{k}-\bar{\theta}_{n}\right) P_{k-1}\left(\widetilde{\theta}_{k}-\bar{\theta}_{n}\right) \underset{n \rightarrow \infty}{\longrightarrow}\left(p+p_{2}\right) \sigma^{2} \text { p.s. }
$$

v) Normalité asymptotique de $\mathfrak{T}_{n}$ et $\mathfrak{S}_{n}$ :

$$
\begin{gathered}
\left\{\sqrt{\frac{1-\alpha}{n^{1-\alpha}}}\left\{\mathfrak{T}_{n}-\frac{n^{1-\alpha}}{1-\alpha}\left(p+p_{2}\right) \sigma^{2}\right\}, \mathcal{G}_{n}\left(\widetilde{\theta}_{n}-\theta\right)\right\} \underset{n \rightarrow \infty}{\stackrel{\mathcal{L}}{\longrightarrow}} \mathfrak{N}\left(0, p_{1} \sigma^{4}\right) \otimes \mathfrak{N}_{p_{1}}\left(0, T(1)^{-1}\right) \otimes \nu \\
\left\{\sqrt{\frac{n^{1-\alpha}}{1-\alpha}}\left\{\mathfrak{S}_{n}-\left(p+p_{2}\right) \sigma^{2}\right\}, \mathcal{G}_{n}\left(\widetilde{\theta}_{n}-\theta\right)\right\} \underset{n \rightarrow \infty}{\stackrel{\mathfrak{L}}{\longrightarrow}} \mathfrak{N}\left(0, p_{1} \sigma^{4}\right) \otimes \mathfrak{N}_{p_{1}}\left(0, T(1)^{-1}\right) \otimes \nu
\end{gathered}
$$

vi) Vitesse de convergence presque-sûre de $\mathfrak{T}_{n}$ et $\mathfrak{S}_{n}$ :

$$
\begin{aligned}
\varlimsup_{n \rightarrow \infty}\left(2 \frac{n^{1-\alpha}}{1-\alpha} \log \log n\right)^{-1 / 2} \mid \mathfrak{T}_{n}-\frac{n^{1-\alpha}}{1-\alpha} & \left(p+p_{2}\right) \sigma^{2} \mid \\
& =\varlimsup_{n \rightarrow \infty}\left(\frac{n^{1-\alpha}}{2(1-\alpha) \log \log n}\right)^{1 / 2}\left|\mathfrak{S}_{n}-\left(p+p_{2}\right) \sigma^{2}\right|=\sqrt{p_{1}} \sigma^{2} p . s .
\end{aligned}
$$

\section{DÉMONSTRATIONS DES PRINCIPAUX RÉSULTATS}

\subsection{Preuves des théorèmes $2.1,3.1,4.1$ et du corollaire 2.2}

Les démonstrations des LFQ énoncés aux paragraphes 2, 3 et 4 sont semblables. C'est pour cette raison qu'elles sont groupées dans cette partie.

Posant : $X_{n}=V_{n}^{-1} \Delta M_{n}$, la relation : $Z_{n+1}=\Lambda_{n} Z_{n}+X_{n+1}$, où $\Lambda_{n}=V_{n+1}^{-1} V_{n}$, implique que

$$
\left\|Z_{n+1}\right\|^{2}=\left\|\Lambda_{n} Z_{n}\right\|^{2}+\left\|X_{n+1}\right\|^{2}+2\left\langle\Lambda_{n} Z_{n}, X_{n+1}\right\rangle ;
$$


d'où l'on déduit l'égalité suivante :

$$
\left\|Z_{n}\right\|^{2}+\sum_{k=0}^{n-1} * Z_{k}\left(I-{ }^{*} \Lambda_{k} \Lambda_{k}\right) Z_{k}=\sum_{k=1}^{n}\left\|X_{k}\right\|^{2}+2 \sum_{k=0}^{n-1}\left\langle\Lambda_{k} Z_{k}, X_{k+1}\right\rangle
$$

De même, on établit l'égalité :

$$
{ }^{*} M_{n} Q_{n}^{-1} M_{n}+\sum_{k=0}^{n-1} * M_{k}\left(Q_{k}^{-1}-Q_{k+1}^{-1}\right) M_{k}=\sum_{k=1}^{n}{ }^{*} \Delta M_{k} Q_{k}^{-1} \Delta M_{k}+2 \sum_{k=0}^{n-1}\left\langle Q_{k+1}^{-1 / 2} M_{k}, Q_{k+1}^{-1 / 2} \Delta M_{k+1}\right\rangle
$$

en notant $Q_{n}=I+\langle M\rangle_{n}$.

Le comportement asymptotique presque-sûr du membre de gauche de (5.1) ainsi que celui de (5.1-bis) sont déterminants pour l'obtention des lois LFQ.

1) Comportement asymptotique des suites $\left(\sum_{k=1}^{n}\left\|X_{k}\right\|^{2}\right),\left(\sum_{k=1}^{n}\left\|Q_{k}^{-1 / 2} \Delta M_{k}\right\|^{2}\right)$.

1-a) Si la martingale $M$ satisfait aux deux hypothèses $H$-1) et $H$-3) ou bien aux deux hypothèses $H$-1) et $H^{\prime}$-3) avec une normalisation $\left(V_{n}\right)$ vérifiant les conditions de croissance régulière $(\mathcal{C})$, alors on a les deux propriétés :

$$
\left(\log \left[\text { Dét } V_{n}\right]^{2}\right)^{-1} \sum_{k=1}^{n}\left\|X_{k}\right\|^{2} \underset{n \rightarrow \infty}{\longrightarrow} \operatorname{tr}\left\{S^{1 / 2} C S^{1 / 2}\right\} \text { p.s. }
$$

et

$$
\left(\log \operatorname{Dét} Q_{n}\right)^{-1} \sum_{k=1}^{n}\left\|Q_{k}^{-1 / 2} \Delta M_{k}\right\|^{2} \underset{n \rightarrow \infty}{\longrightarrow} 1 \text { p.s. sur }\{\text { Dét } C>0\} \text {. }
$$

1-b) Si la martingale $M$ satisfait aux deux hypothèses $H$-1) et $H^{\prime}$-3) avec une normalisation $\left(V_{n}\right)$ vérifiant les conditions de croissance exponentielle $\left(\mathcal{C}^{\prime}\right)$, alors on a les deux propriétés :

$$
\frac{1}{n} \sum_{k=1}^{n}\left\|X_{k}\right\|^{2} \underset{n \rightarrow \infty}{\longrightarrow} \operatorname{tr}\left\{\left(I-{ }^{*} \Lambda_{\infty} \Lambda_{\infty}\right) C\right\} \text { p.s. }
$$

et

$$
\frac{1}{n} \sum_{k=1}^{n}\left\|Q_{k}^{-1 / 2} \Delta M_{k}\right\|^{2} \underset{n \rightarrow \infty}{\longrightarrow} \operatorname{tr}\left(I-C^{-1 / 2} \Lambda_{\infty} C^{*} \Lambda_{\infty} C^{-1 / 2}\right) \text { p.s. } \quad \text { sur }\{\text { Dét } C>0\}
$$

Preuve des assertions 1-a) et 1-b). Supposons d'abord que la martingale $M$ satisfait aux deux hypothèses H-1) et $\left.\mathrm{H}^{\prime}-3\right)$. On a :

$$
\begin{gathered}
\mathbb{E}\left\{\left\|X_{k+1}\right\|^{2} / \mathfrak{F}_{k}\right\}=\operatorname{tr}\left\{V_{k+1}^{-1}\left(\langle M\rangle_{k+1}-\langle M\rangle_{k}\right)^{*} V_{k+1}^{-1}\right\} \\
=\operatorname{tr}\left(C_{k+1}\right)-\operatorname{tr}\left(C_{k}\right)+\operatorname{tr}\left\{C_{k}\left(I-{ }^{*} \Lambda_{k} \Lambda_{k}\right)\right\} \\
\mathbb{E}\left\{\left\|Q_{k+1}^{-1 / 2} \Delta M_{k+1}\right\|^{2} / \mathfrak{F}_{k}\right\}=\operatorname{tr}\left\{Q_{k+1}^{-1 / 2}\left(Q_{k+1}-Q_{k}\right) Q_{k+1}^{-1 / 2}\right\}=f_{k} .
\end{gathered}
$$


Donc les propriétés :

$$
\begin{gathered}
\left(\log \left[\text { Dét } V_{n}\right]^{2}\right)^{-1} \sum_{k=1}^{n} \mathbb{E}\left\{\left\|X_{k}\right\|^{2} / \mathfrak{F}_{k-1}\right\} \underset{n \rightarrow \infty}{\longrightarrow} \operatorname{tr}\left\{S^{1 / 2} C S^{1 / 2}\right\} \text { p.s., } \\
\frac{1}{n} \sum_{k=1}^{n} \mathbb{E}\left\{\left\|X_{k}\right\|^{2} / \mathfrak{F}_{k-1}\right\} \underset{n \rightarrow \infty}{\longrightarrow} \operatorname{tr}\left\{\left(I-{ }^{*} \Lambda_{\infty} \Lambda_{\infty}\right) C\right\} \text { p.s. }
\end{gathered}
$$

sont immédiates si H-1) est vérifiée avec une normalisation $\left(V_{n}\right)$ satisfaisant aux conditions $(\mathcal{C})$ ou $\left(\mathcal{C}^{\prime}\right)$. Le théorème de Chow permet alors de voir, que sous l'hypothèse H'-3), (5.6) [resp (5.7)] implique (5.2) [resp (5.4)].

De même, on montre la propriété (5.3) ou (5.5), car sur $\{$ Dét $C>0\}$, on a :

$\mathrm{ou}$

$$
f_{n} \approx \log \text { Dét } Q_{n+1}-\log \text { Dét } Q_{n} \quad(n \rightarrow \infty)
$$

$$
f_{n} \underset{n \rightarrow \infty}{\longrightarrow} \operatorname{tr}\left(I-C^{-1 / 2} \Lambda_{\infty} C^{*} \Lambda_{\infty} C^{-1 / 2}\right)=f_{\infty}
$$

selon que la normalisation $\left(V_{n}\right)$ vérifie $(\mathcal{C})$ ou bien $\left(\mathcal{C}^{\prime}\right)$. La seconde propriété est immédiate, tandis que la première résulte d'une application du lemme suivant à la suite $\left(Q_{n}^{1 / 2}\right)$ au lieu de $\left(V_{n}\right)$.

Lemme 5.1. Soit $\left(b_{n}\right)$ la suite définie $: b_{n}=\log \left(\frac{\text { Dét } V_{n+1}}{\operatorname{Dét} V_{n}}\right)^{2}$. Sous les conditions $C$-1) et $C$-2), on a les propriétés suivantes.

i) $\operatorname{tr}\left(I-\Lambda_{n}{ }^{*} \Lambda_{n}\right)=a_{n} \leq b_{n} ; \quad$ ii) $a_{n}^{-1} b_{n} \underset{n \rightarrow \infty}{\longrightarrow} 1$;

en conséquence :

$$
a_{n}^{-1}\left[1-\left(\frac{\text { Dét } V_{n}}{\text { Dét } V_{n+1}}\right)^{2}\right] \underset{n \rightarrow \infty}{\longrightarrow} 1 \text { et } \quad\left[\log \left(\text { Dét } V_{N}\right)^{2}\right]^{-1} \sum_{n=1}^{N} a_{n} \underset{N \rightarrow \infty}{\longrightarrow} 1 .
$$

Preuve du lemme 5.1. Soient $\lambda_{n}(1), \cdots, \lambda_{n}(d)$ les valeurs propres de la matrice $\Lambda_{n}{ }^{*} \Lambda_{n}$. On a :

$$
a_{n}=\sum_{j=1}^{d}\left(1-\lambda_{n}(j)\right) \leq-\sum_{j=1}^{d} \log \lambda_{n}(j)=-\log \prod_{j=1}^{d} \lambda_{n}(j) ;
$$

donc : $a_{n} \leq-\log \left(\text { Dét } \Lambda_{n}\right)^{2}=\log \left(\frac{\text { Dét } V_{n+1}}{\text { Dét } V_{n}}\right)^{2}=b_{n}$.

La propriété i) est établie. Par ailleurs, la condition C-2) implique que : $\forall 1 \leq j \leq d, \quad \lambda_{n}(j) \underset{n \rightarrow \infty}{\longrightarrow} 1$ et alors : $-\left[1-\lambda_{n}(j)\right]^{-1} \log \lambda_{n}(j) \underset{n \rightarrow \infty}{\longrightarrow} 1$, pour tout $1 \leq j \leq d$.

Compte tenu de ce qui précède, on déduit que : $a_{n}^{-1} b_{n} \underset{n \rightarrow \infty}{\longrightarrow} 1$.

Vu que : $\left[1-\left(\frac{\text { Dét } V_{n}}{\text { Dét } V_{n+1}}\right)^{2}\right]^{-1} b_{n} \underset{n \rightarrow \infty}{\longrightarrow} 1$, on a les deux dernières propriétés annoncées en ii).

Il reste à établir les propriétés (5.2) et (5.3) lorsque H-1) et H-3) sont satisfaites et la normalisation $\left(V_{n}\right)$ vérifie $(\mathcal{C})$. La validité de $(5.2)$ sous ces hypothèses résulte du lemme 5.1 et de la relation :

$$
\begin{aligned}
\left\|X_{k+1}\right\|^{2} & =\operatorname{tr}\left\{V_{k+1}^{-1} \Delta M_{k+1}{ }^{*} \Delta M_{k+1}{ }^{*} V_{k+1}\right\} \\
& =\operatorname{tr}\left(V_{k+1}^{-1}[M]_{k+1}{ }^{*} V_{k+1}^{-1}\right)-\operatorname{tr}\left(V_{k}^{-1}[M]_{k}{ }^{*} V_{k}^{-1}\right)+\operatorname{tr}\left\{V_{k}^{-1}[M]_{k}{ }^{*} V_{k}^{-1}\left(I-{ }^{*} \Lambda_{k} \Lambda_{k}\right)\right\} .
\end{aligned}
$$


La propriété (5.3) est une conséquence de (5.2), car sur $\{$ Dét $C>0\}$ :

$\left\|Q_{k+1}^{-1 / 2} \Delta M_{k+1}\right\|^{2}={ }^{*} X_{k+1}{ }^{*} V_{k+1} Q_{k+1}^{-1} V_{k+1} X_{k+1}={ }^{*} X_{k+1} C^{-1} X_{k+1}+o\left(\left\|X_{k+1}\right\|^{2}\right)$

et on a :

$$
\begin{aligned}
{ }^{*} X_{k+1} C^{-1} X_{k+1}=\operatorname{tr}\left\{C^{-1} V_{k+1}^{-1}[M]_{k+1}{ }^{*} V_{k+1}^{-1}\right\} & -\operatorname{tr}\left\{C^{-1} V_{k}^{-1}[M]_{k}{ }^{*} V_{k}^{-1}\right\} \\
& +\operatorname{tr}\left\{C^{-1 / 2} V_{k}^{-1}[M]_{k}{ }^{*} V_{k}^{-1} C^{-1 / 2}\left(I-C^{1 / 2}{ }^{*} \Lambda_{k} C^{-1} \Lambda_{k} C^{1 / 2}\right)\right\} ;
\end{aligned}
$$

donc :

avec :

$$
\sum_{k=1}^{n}\left\|Q_{k}^{-1 / 2} \Delta M_{k}\right\|^{2}=\sum_{k=0}^{n-1} a_{k}^{\prime}+o\left(\sum_{k=0}^{n-1} a_{k}^{\prime}\right)+o\left(\log \left(\text { Dét } V_{n}\right)^{2}\right)+\operatorname{tr}\left\{C^{-1} V_{n+1}^{-1}[M]_{n+1}{ }^{*} V_{n+1}^{-1}\right\}
$$

$$
a_{k}^{\prime}=\operatorname{tr}\left\{I-C^{1 / 2 *} \Lambda_{k} C^{-1} \Lambda_{k} C^{1 / 2}\right\} .
$$

En appliquant le lemme 5.1 aux matrices $V_{n}^{\prime}=C^{-1 / 2} V_{n} C^{1 / 2}$ sur $\{$ Dét $C>0\}$, on peut affirmer que sur cet ensemble :

$$
\sum_{k=0}^{n-1} a_{k}^{\prime} \simeq \log \left(\text { Dét } V_{n}\right)^{2} \quad(n \rightarrow \infty) .
$$

Or, sur $\{$ Dét $C>0\}$, l'hypothèse H-1) implique que :

$$
\frac{\log \text { Dét } Q_{n}}{\log \left(\text { Dét } V_{n}\right)^{2}} \underset{n \rightarrow \infty}{\longrightarrow} 1 \text { p.s. ; }
$$

donc les assertions 1-a) et 1-b) sont établies.

2) Comportement asymptotique de la martingale $\sum_{k=0}^{n-1}\left\langle\Lambda_{k} Z_{k}, X_{k+1}\right\rangle$

Au début de cette partie, on se propose d'établir que, dans le cadre des théorèmes 2.1 et 3.1, la martingale $L_{n}=\sum_{k=0}^{n-1}\left\langle\Lambda_{k} Z_{k}, X_{k+1}\right\rangle$ vérifie :

$$
\left(\log \left[\operatorname{Dét} V_{n}\right]^{2}\right)^{-1} L_{n} \underset{n \rightarrow \infty}{\longrightarrow} 0 \text { p.s. }
$$

Autrement dit les suites : $\left(\left[\log \left[\operatorname{Dét} V_{n}\right]^{2}\right]^{-1} \sum_{k=1}^{n}\left\|X_{k}\right\|^{2}\right)$ et $\left(\left[\log \left[\operatorname{Dét} V_{n}\right]^{2}\right]^{-1}\left\{\left\|Z_{n}\right\|^{2}+\sum_{k=0}^{n-1}{ }^{*} Z_{k}\left(I-{ }^{*} \Lambda_{k} \Lambda_{k}\right) Z_{k}\right\}\right)$
convergent p.s. vers la même limite.

$\mathrm{Vu}$ que la variation quadratique prévisible de la martingale $\left(L_{n}\right)$ vaut :

la propriété :

$$
\langle L\rangle_{n}=\sum_{k=0}^{n-1} * Z_{k}{ }^{*} \Lambda_{k} V_{k+1}^{-1}\left(\langle M\rangle_{k+1}-\langle M\rangle_{k}\right){ }^{*} V_{k+1}^{-1} \Lambda_{k} Z_{k}
$$

$$
\langle L\rangle_{n}=O\left(\left\|Z_{n}\right\|^{2}+\sum_{k=0}^{n-1}{ }^{*} Z_{k}\left(I-{ }^{*} \Lambda_{k} \Lambda_{k}\right) Z_{k}+\sum_{k=1}^{n}\left\|X_{k}\right\|^{2}\right) \quad \text { p.s. }(n \rightarrow \infty)
$$


est essentielle pour l'obtention de (5.8) et des LFQ. Montrons qu'elle a lieu dans les deux cas suivants :

2-c) $M$ satisfait l'hypothèse $H$-1) avec une suite $\left(V_{n}\right)$ vérifiant les conditions $(\mathcal{C})$;

2-d) $M$ satisfait l'hypothèse $H-1)$ avec une suite $\left(V_{n}\right)$ vérifiant les conditions $\left(\mathcal{C}^{\prime}\right)$.

Preuve de la propriété (5.8-bis) dans les deux cas 2-c) et 2-d). On a :

$$
\begin{aligned}
\langle L\rangle_{n} & =O\left(\sum_{k=0}^{n-1}\left\|Z_{k}\right\|^{2} \operatorname{tr}\left(C_{k+1}-\Lambda_{k} C_{k}{ }^{*} \Lambda_{k}\right)\right) \\
& =O\left(\left|\sum_{k=0}^{n-1}\left\|Z_{k}\right\|^{2}\left[\operatorname{tr}\left(C_{k+1}\right)-\operatorname{tr}\left(C_{k}\right)\right]\right|\right)+O\left(\sum_{k=0}^{n-1}\left\|Z_{k}\right\|^{2} \operatorname{tr}\left\{\left(I-{ }^{*} \Lambda_{k} \Lambda_{k}\right) C_{k}\right\}\right) \text { p.s. },(n \rightarrow \infty) .
\end{aligned}
$$

Il est donc immédiat que la propriété (5.8-bis) a lieu dans le cas 2-d). Pour montrer qu'elle est vraie dans le cas 2-c), il suffit d'établir que, sur $\Omega_{\infty}=\left\{\langle L\rangle_{\infty}=\lim \uparrow\langle L\rangle_{n}=\infty\right\}$, on a :

$$
J_{n}=\sum_{k=0}^{n-1}\left\|Z_{k}\right\|^{2}\left[\operatorname{tr}\left(C_{k+1}\right)-\operatorname{tr}\left(C_{k}\right)\right]=O\left(I_{n}\right)+o\left(\langle L\rangle_{n}\right) \text { p.s. }(n \rightarrow \infty)
$$

avec : $I_{n}=\left\|Z_{n}\right\|^{2}+\sum_{k=0}^{n-1} * Z_{k}\left(I-{ }^{*} \Lambda_{k} \Lambda_{k}\right) Z_{k}+\sum_{k=1}^{n}\left\|X_{k}\right\|^{2}$.

Or :

$$
J_{n}=\operatorname{tr}\left(C_{n}\right)\left\|Z_{n}\right\|^{2}-\sum_{k=0}^{n-1} \operatorname{tr}\left(C_{k+1}\right)\left[\left\|Z_{k+1}\right\|^{2}-\left\|Z_{k}\right\|^{2}\right]
$$

et

$$
\left\|Z_{k+1}\right\|^{2}-\left\|Z_{k}\right\|^{2}=-{ }^{*} Z_{k}\left(I-{ }^{*} \Lambda_{k} \Lambda_{k}\right) Z_{k}+\left\|X_{k+1}\right\|^{2}+2\left\langle\Lambda_{k} Z_{k}, X_{k+1}\right\rangle ;
$$

donc :

$$
\left|J_{n}\right|=O\left(I_{n}\right)+O\left(\left|L_{n}^{\prime}\right|\right) \quad \text { p.s. }
$$

avec :

Vu que :

$$
L_{n}^{\prime}=\sum_{k=0}^{n-1}\left[\operatorname{tr}\left(C_{k+1}\right)\right]\left\langle\Lambda_{k} Z_{k}, X_{k+1}\right\rangle
$$

$$
\left\langle L^{\prime}\right\rangle_{n}=\sum_{k=0}^{n-1}\left[\operatorname{tr}\left(C_{k+1}\right)\right]^{2}\left(\langle L\rangle_{k+1}-\langle L\rangle_{k}\right)=O\left(\langle L\rangle_{n}\right) \text { p.s. },(n \rightarrow \infty),
$$

la loi forte des grands nombres pour les martingales scalaires implique que, sur $\Omega_{\infty}$, on a :

$$
\left|J_{n}\right|=O\left(I_{n}\right)+o\left(\langle L\rangle_{n}\right) \text { p.s. }(n \rightarrow \infty) .
$$

En conséquence :

$$
\langle L\rangle_{n}=O\left(I_{n}\right)+o\left(\langle L\rangle_{n}\right)=O\left(I_{n}\right) \text { p.s., }(n \rightarrow \infty)
$$

$\operatorname{sur} \Omega_{\infty}$. La propriété (5.8-bis) est établie.

3) Preuves des LFQ sous les conditions $(\mathcal{C})$ ou $\left(\mathcal{C}^{\prime}\right)$

Supposons que $M$ vérifie les hypothèses considérées en 1-a), alors les propriétés 5.1, 5.2, 5.8-bis et la loi des grands nombres pour les martingales scalaires impliquent que p.s. :

$$
\left(\log \left[\operatorname{Dét} V_{n}\right]^{2}\right)^{-1}\left(\left\|Z_{n}\right\|^{2}+\sum_{k=1}^{n}{ }^{*} Z_{k}\left(I-{ }^{*} \Lambda_{k} \Lambda_{k}\right) Z_{k}\right) \underset{n \rightarrow \infty}{\longrightarrow} \operatorname{tr}\left\{S^{\frac{1}{2}} C S^{\frac{1}{2}}\right\} .
$$


On en déduit grâce à C-3) et au lemme 5.1 :

$$
\lim _{n \rightarrow \infty} \frac{1}{\log \left[\text { Dét } V_{n}\right]^{2}}\left(\left\|Z_{n}\right\|^{2}+\sum_{k=1}^{n}\left[1-\left(\frac{\text { Dét } V_{k}}{\text { Dét } V_{k+1}}\right)^{2}\right]{ }^{*} Z_{k} S Z_{k}\right)=\operatorname{tr}\left\{S^{\frac{1}{2}} C S^{\frac{1}{2}}\right\} \quad \text { p.s. }
$$

Sous les hypothèses considérées en 1-a), on a également $(c f .[10,14])$ :

$$
\lim _{n \rightarrow \infty} \frac{1}{\log \operatorname{Dét} Q_{n}}\left({ }^{*} M_{n} Q_{n}^{-1} M_{n}+\sum_{k=1}^{n}{ }^{*} M_{k}\left(Q_{k}^{-1}-Q_{k+1}^{-1}\right) M_{k}\right)=1 \text { sur }\{\text { Dét } C>0\} .
$$

En effet, dans la relation (5.1-bis), la variation quadratique prévisible de la martingale $\widetilde{L}_{n}=\sum_{k=0}^{n-1}\left\langle Q_{k+1}^{-1 / 2} M_{k}, Q_{k+1}^{-1 / 2} \Delta M_{k+1}\right\rangle$ vérifie :

$$
\langle\widetilde{L}\rangle_{n}=\sum_{k=0}^{n-1} * M_{k} Q_{k+1}^{-1}\left(Q_{k+1}-Q_{k}\right) Q_{k+1}^{-1} M_{k} \leq \sum_{k=0}^{n-1} * M_{k}\left(Q_{k}^{-1}-Q_{k+1}^{-1}\right) M_{k} ;
$$

donc (5.3) et la loi forte des grands nombres pour les martingales scalaires impliquent (5.10-bis).

Par ailleurs, lorsque les deux hypothèses $\mathrm{H}-1$ ) et $\mathrm{H}-2)$ ou $\mathrm{H}-1$ ) et $\mathrm{H}$-2) sont vérifiées avec une suite ( $\left.V_{n}\right)$ satisfaisant les conditions $(\mathcal{C})$, on a $(c f$. Th. I de l'annexe) :

$$
\frac{1}{\log \left[\text { Dét } V_{n}\right]^{2}} \sum_{k=1}^{n}\left[1-\left(\frac{\text { Dét } V_{k}}{\text { Dét } V_{k+1}}\right)^{2}\right] \delta_{Z_{k}} \Rightarrow \mu_{\infty} \quad \text { p.s. ; }
$$

donc la minoration ( $c f$. Cor. IV de l'annexe) :

$$
\varliminf_{n \rightarrow \infty} \frac{1}{\log \left[\text { Dét } V_{n}\right]^{2}} \sum_{k=1}^{n}\left[1-\left(\frac{\text { Dét } V_{k}}{\text { Dét } V_{k+1}}\right)^{2}\right] * Z_{k} S Z_{k} \geq \operatorname{tr}\left\{S^{\frac{1}{2}} C S^{\frac{1}{2}}\right\} \quad \text { p.s. }
$$

a lieu, à condition que $\mu_{\infty}$ soit de covariance $C$ : l'hypothèse H-4) est vérifiée (ce qui est évidemment le cas sous H-1) et H'-2)). En comparant (5.10) et (5.12), on obtient :

$$
\lim _{n \rightarrow \infty} \frac{\left\|Z_{n}\right\|^{2}}{\log \left[\operatorname{Dét} V_{n}\right]^{2}}=0 \quad \text { p.s. }
$$

et

$$
\lim _{n \rightarrow \infty} \frac{1}{\log \left[\text { Dét } V_{n}\right]^{2}} \sum_{k=1}^{n}\left[1-\left(\frac{\text { Dét } V_{k}}{\text { Dét } V_{k+1}}\right)^{2}\right] * Z_{k} S Z_{k}=\operatorname{tr}\left\{S^{\frac{1}{2}} C S^{\frac{1}{2}}\right\} \quad \text { p.s. }
$$

Mais sur $\{$ Dét $C>0\}$, l'hypothèse H-1) et (5.13) impliquent :

$$
\left(\operatorname{LogDét} Q_{n}\right)^{-1}{ }^{*} M_{n} Q_{n}^{-1} M_{n} \underset{n \rightarrow \infty}{\longrightarrow} 0 \text { p.s. ; }
$$


donc compte tenu de $\mathrm{H}-1)$ et (5.10-bis) :

$$
\lim _{n \rightarrow \infty} \frac{1}{\log \left[\text { Dét } V_{n}\right]^{2}} \sum_{k=1}^{n} * M_{k}\left(Q_{k}^{-1}-Q_{k+1}^{-1}\right) M_{k}=1 \quad \text { p.s. }
$$

La matrice $S$ étant régulière, il est classique que (5.11) et (5.14) impliquent les lois fortes quadratique et logarithmique. Le théorème 2.1 et le corollaire 2.2 sont établis sauf dans le cadre de la partie 3 ) du théorème 2.1, cas particulier où les matrices $\left(V_{n}\right)$ sont diagonales, examiné ci-dessous en 4$)$.

Dans le cadre 1-b) (qui contient 2-d)), on montre de même les propriétés suivantes analogues à (5.10) et (5.10-bis) :

$$
\lim _{n \rightarrow \infty} \frac{1}{n}\left(\left\|Z_{n}\right\|^{2}+\sum_{k=0}^{n-1}{ }^{*} Z_{k}\left(I-{ }^{*} \Lambda_{k} \Lambda_{k}\right) Z_{k}\right)=\operatorname{tr}\left\{\left(I-{ }^{*} \Lambda_{\infty} \Lambda_{\infty}\right) C\right\} \text { p.s. }
$$

et

$$
\begin{aligned}
\lim _{n \rightarrow \infty} \frac{1}{n}\left({ }^{*} M_{n} Q_{n}^{-1} M_{n}+\sum_{k=0}^{n-1} * M_{k}\right. & \left.\left(Q_{k}^{-1}-Q_{k+1}^{-1}\right) M_{k}\right) \\
& =\operatorname{tr}\left(I-C^{-1 / 2} \Lambda_{\infty} C^{*} \Lambda_{\infty} C^{-1 / 2}\right) \quad \text { p.s. sur }\{\text { Dét C }>0\} .
\end{aligned}
$$

Si de plus l'hypothèse H-2) est satisfaite, la propriété (5.15), le TLCPSG ( $c f$. Th. II de l'annexe) :

$$
\frac{1}{n} \sum_{k=1}^{n} \delta_{Z_{k}} \underset{n \rightarrow \infty}{\Longrightarrow} \mu_{\infty} \text { p.s. }
$$

et le fait que $\mu_{\infty}$ soit de covariance $C$ impliquent comme ci-dessus :

$$
\lim _{n \rightarrow \infty} \frac{\left\|Z_{n}\right\|^{2}}{n}=0 \text { p.s. }
$$

et

$$
\lim _{n \rightarrow \infty} \frac{1}{n} \sum_{k=1}^{n}{ }^{*} Z_{k}\left(I-{ }^{*} \Lambda_{\infty} \Lambda_{\infty}\right) Z_{k}=\operatorname{tr}\left\{\left(I-{ }^{*} \Lambda_{\infty} \Lambda_{\infty}\right) C\right\} \text { p.s. }
$$

Partant de (5.16), on vérifie aisément la dernière assertion du théorème 3.1 .

Grâce à (5.17) et au TLCPSG, on peut affirmer que :

et cette propriété implique :

$$
\frac{1}{n} \sum_{k=1}^{n} Z_{k} * Z_{k} \underset{n \rightarrow \infty}{\longrightarrow} C \text { p.s. }
$$

$$
\lim _{n \rightarrow \infty} A_{n}^{-1}(1) \sum_{k=1}^{n} a_{k}(1) Z_{k}{ }^{*} Z_{k}=C \text { p.s. }
$$

pour toute suite $\left(a_{n}(1)\right)$ satisfaisant (4.2) (cf. Sect. 4). 
Sur $\{$ Dét $C>0\},(5.15$-bis) et (5.16) impliquent :

$$
\frac{1}{n} \sum_{k=1}^{n-1} M_{k}\left(Q_{k}^{-1}-Q_{k+1}^{-1}\right) M_{k} \underset{n \rightarrow \infty}{\longrightarrow} \operatorname{tr}\left(I-C^{-1 / 2} \Lambda_{\infty} C^{*} \Lambda_{\infty} C^{-1 / 2}\right) \text { p.s. }
$$

Le théorème 3.1 est établi.

\section{4) Preuve de la partie 3) du théorème 2.1}

Les propriétés (5.2) et (5.9) [resp (5.4) et (5.15)] que l'on vient d'établir sous les hypothèses considérées en 1-a) [resp 1-b)] impliquent évidemment (5.8). Montrons que cette propriété est encore vraie si les hypothèses $\mathrm{H}-1$ ) et H'-3) sont vérifiées avec une normalisation diagonale.

En effet, si $V_{n}=\operatorname{Diag}\left(v_{n}(1), \cdots v_{n}(d)\right)$, l'égalité (5.1) est la somme des égalités suivantes :

$$
\frac{M_{n}^{2}(j)}{v_{n}^{2}(j)}+\sum_{k=1}^{n-1}\left[1-\left(\frac{v_{k}(j)}{v_{k+1}(j)}\right)^{2}\right] \frac{M_{k}^{2}(j)}{v_{k}^{2}(j)}=\sum_{k=1}^{n} \frac{\left(\Delta M_{k}(j)\right)^{2}}{v_{k}^{2}(j)}+2 \sum_{k=0}^{n-1} \frac{M_{k}(j)}{v_{k+1}^{2}(j)} \Delta M_{k+1}(j)
$$

où $M(j)=\left(M_{n}(j)\right)$ est la martingale scalaire : $M_{n}(j)=\left\langle e_{j}, M_{n}\right\rangle, \quad j=1, \cdots, d$.

La transcription de (5.1-bis) pour cette martingale est :

$$
\frac{M_{n}^{2}(j)}{\langle M(j)\rangle_{n}}+\sum_{k=1}^{n-1}\left[1-\frac{\langle M(j)\rangle_{k}}{\langle M(j)\rangle_{k+1}}\right] \frac{M_{k}^{2}(j)}{\langle M(j)\rangle_{k}}=\sum_{k=1}^{n} \frac{\left(\Delta M_{k}(j)\right)^{2}}{\langle M(j)\rangle_{k}}+2 \sum_{k=0}^{n-1} \frac{M_{k}(j)}{\langle M(j)\rangle_{k+1}} \Delta M_{k+1}(j) .
$$

Sous l'hypothèse H'-3), la martingale $M(j)$ vérifie la relation (5.10-bis), à savoir, p.s. :

$$
\lim _{n \rightarrow \infty}\left[\log \langle M(j)\rangle_{n}\right]^{-1}\left[\frac{M_{n}^{2}(j)}{\langle M(j)\rangle_{n}}+\sum_{k=0}^{n-1}\left(1-\frac{\langle M(j)\rangle_{k}}{\langle M(j)\rangle_{k+1}}\right) \frac{M_{k}^{2}(j)}{\langle M(j)\rangle_{k}}\right]=1
$$

$\operatorname{sur}\left\{\log \langle M(j)\rangle_{n} \longrightarrow \infty\right\} \quad(c f$. [14,24]). Or dans l'égalité (5.19), la variation quadratique prévisible de la martingale $L(j)=\left(L_{n}(j)\right)$ définie par :

vaut :

$$
L_{n}(j)=\sum_{k=0}^{n-1} \frac{M_{k}(j)}{v_{k+1}^{2}(j)} \Delta M_{k+1}(j)
$$

$$
\langle L(j)\rangle_{n}=\sum_{k=0}^{n-1} \frac{M_{k}^{2}(j)}{\langle M(j)\rangle_{k}} \frac{\langle M(j)\rangle_{k}}{v_{k+1}^{2}(j)} \frac{\langle M(j)\rangle_{k+1}}{v_{k+1}^{2}(j)}\left[1-\frac{\langle M(j)\rangle_{k}}{\langle M(j)\rangle_{k+1}}\right] ;
$$

il est donc immédiat que sous H-1) et H'-3) :

$$
\langle L(j)\rangle_{n}=O\left(\log v_{n}^{2}(j)\right) \quad \text { p.s. }(n \rightarrow \infty) .
$$

On en déduit (5.8), car la condition C-3) et le lemme 5.1 impliquent :

$$
\log v_{n}^{2}(j) \approx{ }^{*} e_{j} S e_{j} \log \left[\text { Dét } V_{n}\right]^{2} \quad(n \rightarrow \infty) .
$$

Par ailleurs, sous les hypothèses H-1) et H'-3), on a :

$$
M_{n}(j)=o\left(\sqrt{v_{n}^{2}(j) \log v_{n}^{2}(j)}\right) \quad \text { p.s., } \forall 1 \leq j \leq d
$$

$(c f .[12,22])$; donc ii) est vérifiée. On en déduit i) et iii) car sous ces hypothèses la propriété (5.10) a lieu. La partie 3$)$ du théorème 2.1 est établie. 


\section{5) Étude du cas mixte du théorème 4.1}

Supposons enfin que la martingale $M=\left(M_{n}(1), M_{n}(2)\right)$ satisfait les hypothèses H-1), H-2) et H-4) avec une normalisation $\left(V_{n}\right)$ de la forme : $V_{n}=\operatorname{Diag}\left\{V_{n}(1), V_{n}(2)\right\}$ et satisfaisant (4.2). Et que le couple $(M(j), V(j)), j=$ 1,2 , vérifie l'hypothèse $\left.\mathrm{H}^{\prime}-3\right)$. Alors d'après le théorème II de l'annexe, on a :

(TLCPSG)

$$
\frac{1}{A_{N}(1)} \sum_{n=1}^{N} a_{n}(1) \delta_{\left(Z_{n}(1), Z_{n}(2)\right)} \underset{n \rightarrow \infty}{\Longrightarrow} \mu_{\infty} \quad \text { p.s. }
$$

en notant :

$$
Z_{n}(j)=V_{n}^{-1}(j) M_{n}(j) \quad \text { pour } \quad j=1 \text { ou } 2 .
$$

Comme $\mu_{\infty}$ est de covariance $C$ (i.e. l'hypothèse $\mathrm{H}-4$ ) est vérifiée), les lois fortes quadratiques établies précédemment et (4.2) impliquent :

$$
A_{N}^{-1}(1) \sum_{n=1}^{N} a_{n}(1)\left(\left\|Z_{n}(1)\right\|^{2}+\left\|Z_{n}(2)\right\|^{2}\right) \underset{N \rightarrow \infty}{\longrightarrow} \operatorname{tr}(C) \text { p.s. }
$$

De cette propriété et du TLCPSG résulte la validité de la loi forte quadratique corres-pondant au cadre mixte du théorème 4.1. La dernière propriété de ce théorème découle des deux résultats suivants valables presquesûrement sur $\{$ Dét $C>0\}$, lesquels s'obtiennent aisément à partir de l'analyse des relations (5.1) et (5.1-bis) dans le cadre mixte envisagé :

$$
\lim _{n \rightarrow \infty} n^{-1}\left({ }^{*} M_{n} Q_{n}^{-1} M_{n}+\sum_{k=0}^{n-1}{ }^{*} M_{k}\left(Q_{k}^{-1}-Q_{k+1}^{-1}\right) M_{k}\right)=\operatorname{tr}\left(I-C^{-1 / 2}(2) \Lambda_{\infty}(2) C(2){ }^{*} \Lambda_{\infty}(2) C^{-1 / 2}(2)\right)
$$

et

$$
\lim _{n \rightarrow \infty} n^{-1 *} M_{n} Q_{n}^{-1} M_{n}=0
$$

\subsection{Preuves des théorèmes $2.3-2.5,3.2$ et 3.3}

\subsubsection{TLC avec poids}

Cette partie est consacrée aux démonstrations des TLC correspondants aux LFQ énoncées aux paragraphes 2 et 3 .

\section{a) Deux relations fondamentales}

Un calcul simple montre que :

$$
\begin{array}{r}
V_{n+1}^{-1}\left(M_{n+1}{ }^{*} M_{n+1}-[M]_{n+1}\right){ }^{*} V_{n+1}^{-1}+\sum_{k=0}^{n}\left(V_{k}^{-1}\left\{M_{k}{ }^{*} M_{k}-[M]_{k}\right\}{ }^{*} V_{k}^{-1}-V_{k+1}^{-1}\left\{M_{k}{ }^{*} M_{k}-[M]_{k}\right\}{ }^{*} V_{k+1}^{-1}\right) \\
=H_{n+1}+{ }^{*} H_{n+1}
\end{array}
$$

avec $H_{n+1}=\sum_{k=0}^{n} V_{k+1}^{-1} \quad M_{k}^{*}\left(V_{k+1}^{-1} \Delta M_{k+1}\right)$.

En particulier, si la normalisation $V_{n}$ est scalaire : $V_{n}=v_{n} I$, alors (5.23) s'écrit :

$$
v_{n+1}^{-2}\left(M_{n+1}{ }^{*} M_{n+1}-[M]_{n+1}\right)+\sum_{k=0}^{n}\left\{1-\left(\frac{v_{k}}{v_{k+1}}\right)^{2}\right\} v_{k}^{-2}\left(M_{k}{ }^{*} M_{k}-[M]_{k}\right)=K_{n+1}+{ }^{*} K_{n+1}
$$

avec $K_{n+1}=\sum_{k=0}^{n} v_{k+1}^{-2} M_{k}^{*}\left(\Delta M_{k+1}\right)$. 
Dans l'égalité (5.23), la suite $\left(H_{n}^{u}\right)$ définie pour $u \in \mathbb{R}^{d}$ par :

$$
H_{n+1}^{u}=H_{n+1} u=\sum_{k=0}^{n} \Lambda_{k} V_{k}^{-1} M_{k}\left\langle\Delta M_{k+1},{ }^{*} V_{k+1}^{-1} u\right\rangle=\sum_{k=0}^{n} \Lambda_{k} Z_{k}\left\langle X_{k+1}, u\right\rangle
$$

est une martingale vectorielle de variation quadratique prévisible :

$$
\left\langle H^{u}\right\rangle_{n+1}=\sum_{k=0}^{n} \Lambda_{k} V_{k}^{-1} M_{k}{ }^{*} M_{k}{ }^{*} V_{k}^{-1}{ }^{*} \Lambda_{k}\left({ }^{*} u V_{k+1}^{-1}\left(\langle M\rangle_{k+1}-\langle M\rangle_{k}\right){ }^{*} V_{k+1}^{-1} u\right)
$$

Tandis que dans $(5.24), K^{u, y}=\left({ }^{*} u K_{n} y\right)$ est, pour $u, y$ dans $\mathbb{R}^{d}$, une martingale scalaire de variation quadratique prévisible :

$$
\left\langle K^{u, y}\right\rangle_{n+1}=\sum_{k=0}^{n} \frac{\left\langle u, M_{k}\right\rangle^{2}}{v_{k+1}^{4}}{ }^{*} y\left(\langle M\rangle_{k+1}-\langle M\rangle_{k}\right) y
$$

Les convergences en loi données aux théorèmes $2.3,2.5$ et 3.2 reposent sur les lemmes suivants et le TLCG rappelé en 1.3 .

Pour $u_{1}, \ldots, u_{d}, x_{1}, \ldots, x_{d} 2 \mathrm{~d}$ vecteurs de $\mathbb{R}^{d}$, posons :

où

$$
H_{n}^{\prime}=\sum_{j=1}^{d}\left\langle x_{j}, H_{n}^{j}\right\rangle, \quad K_{n}^{\prime}=\sum_{j=1}^{d}\left\langle u_{j}, K_{n}^{j}\right\rangle
$$

$$
H_{n}^{j}=H_{n} e_{j}, \quad K_{n}^{j}=K_{n} e_{j} .
$$

b) Comportement asymptotique des suites $\left(\left\langle H^{\prime}\right\rangle_{n}\right),\left(\left\langle K^{\prime}\right\rangle_{n}\right)$.

Lemme 5.2. Si la martingale $M=\left(M_{n}\right)_{n \geq 0}$ satisfait l'hypothèse $H$-1) avec une normalisation $\left(V_{n}\right)$ satisfaisant les conditions de croissance régulière $C$-1), $C$-2) et $C$-3-bis), alors pour tout $r>0$ :

$$
\exp \left(-r A_{N}\right) \sum_{n=1}^{N}\left[\exp \left(r A_{n}\right)-\exp \left(r A_{n-1}\right)\right] a_{n}^{-1} V_{n+1}^{-1}\left(\langle M\rangle_{n+1}-\langle M\rangle_{n}\right){ }^{*} V_{n+1}^{-1} \underset{N \rightarrow \infty}{\longrightarrow} \widetilde{C} \text { p.s. }
$$

ò̀ $\widetilde{C}=U C+C^{*} U ; \quad U$ étant la matrice introduite dans C-3-bis).

Preuve du lemme 5.2. Posant: $b_{k}^{-1}=\exp \left(r A_{k}\right)-\exp \left(r A_{k-1}\right)$ et

$$
F_{n}(u)=\sum_{k=1}^{n} b_{k}^{-1} a_{k}^{-1 *} u V_{k+1}^{-1}\left(\langle M\rangle_{k+1}-\langle M\rangle_{k}\right){ }^{*} V_{k+1}^{-1} u
$$

on a :

$$
F_{n}(u)=F_{n}^{\prime}(u)+F_{n}^{\prime \prime}(u)
$$

avec :

$$
\begin{aligned}
& F_{n}^{\prime}(u)=\sum_{k=1}^{n} b_{k}^{-1} a_{k}^{-1} * u\left(C_{k}-\Lambda_{k} C_{k} * \Lambda_{k}\right) u \text { et } F_{n}^{\prime \prime}(u)=\sum_{k=1}^{n} b_{k}^{-1} a_{k}^{-1 *} u\left(C_{k+1}-C_{k}\right) u \\
& \left(\begin{array}{l}
a_{0}=A_{0} \\
\text { Vu que }
\end{array}\right) .
\end{aligned}
$$

$$
C_{k}-\Lambda_{k} C_{k}^{*} \Lambda_{k}=a_{k}\left(U C_{k}+C_{k}^{*} U\right)+o\left(a_{k}\right)
$$


l'hypothèse $\mathrm{H}-1$ ) permet d'affirmer que :

$$
a_{k}^{-1}\left(C_{k}-\Lambda_{k} C_{k}{ }^{*} \Lambda_{k}\right) \underset{k \rightarrow \infty}{\longrightarrow} U C+C{ }^{*} U=\widetilde{C} .
$$

D'où l'on déduit que :

$$
\exp \left(-r A_{n}\right) F_{n}^{\prime}(u) \underset{n \rightarrow \infty}{\longrightarrow} * u \widetilde{C} u \text { p.s., } \forall u \in \mathbb{R}^{d}
$$

Par ailleurs, en écrivant $F_{n}^{\prime \prime}(u)$ sous la forme :

$$
\begin{aligned}
F_{n}^{\prime \prime}(u) & =\sum_{k=1}^{n}\left(b_{k+1}^{-1} a_{k+1}^{-1}{ }^{*} u C_{k+1} u-b_{k}^{-1} a_{k}^{-1}{ }^{*} u C_{k} u\right)-\sum_{k=1}^{n}\left(b_{k+1}^{-1} a_{k+1}^{-1}-b_{k}^{-1} a_{k}^{-1}\right){ }^{*} u C_{k+1} u \\
& =b_{n+1}^{-1} a_{n+1}^{-1}{ }^{*} u C_{n+1} u-\sum_{k=1}^{n}\left(b_{k+1}^{-1} a_{k+1}^{-1}-b_{k}^{-1} a_{k}^{-1}\right){ }^{*} u C_{k+1} u-b_{1}^{-1} a_{1}^{-1}{ }^{*} u C_{1} u
\end{aligned}
$$

et en remarquant que $b_{k+1}^{-1} a_{k+1}^{-1}-b_{k}^{-1} a_{k}^{-1} \geq 0$ pour $k$ assez grand, on déduit, grâce à H-1) et au lemme de Toeplitz :

$$
b_{n+1} a_{n+1} F_{n}^{\prime \prime}(u) \underset{n \rightarrow \infty}{\longrightarrow} 0 \text { p.s. }
$$

Comme on a :

$$
\begin{aligned}
b_{n+1} a_{n+1} \exp \left(r A_{n}\right) & =a_{n+1} \frac{\exp \left(r A_{n}\right)}{\exp \left(r A_{n+1}\right)-\exp \left(r A_{n}\right)} \\
& =\frac{a_{n+1}}{\exp \left(r a_{n+1}\right)-1} \underset{n \rightarrow \infty}{\longrightarrow} \frac{1}{r},
\end{aligned}
$$

on obtient l'assertion du lemme, car d'après (5.27-5.29) et (5.30) pour tout $u \in \mathbb{R}^{d}$ :

$$
\exp \left(-r A_{n}\right) F_{n}(u)=\exp \left(-r A_{n}\right) F_{n}^{\prime}(u)+\frac{b_{n+1} a_{n+1}}{b_{n+1} a_{n+1} \exp \left(r A_{n}\right)} F_{n}^{\prime \prime}(u) \underset{n \rightarrow \infty}{\longrightarrow} * u \widetilde{C} u \text { p.s. }
$$

\section{Lemme 5.3.}

1) Si la martingale $M=\left(M_{n}\right)_{n \geq 0}$ satisfait les hypothèses $\{H-1), H$-2) , $[H-3)$ ou $H^{\prime}$-3)] et $H$-4)\} avec une normalisation $\left(V_{n}\right)$ satisfaisant aux conditions de croissance régulière $(\mathcal{C})$, alors :

$$
\frac{\left\langle H^{u}\right\rangle_{n}}{A_{n}} \underset{n \rightarrow \infty}{\longrightarrow}\left({ }^{*} u \widetilde{C} u\right) C \text { p.s. }
$$

Ce résultat est également vrai en substituant l'hypothèse $\left.H^{\prime}-2\right)$ au couple $\{H-2), H$-4)\}.

2) Si $M$ satisfait les hypothèses $\{H-1), H$-2), [H-3) ou $\left.\left.H^{\prime}-3\right)\right]$ et $H$-4) $\}$ ou bien $\left.\{H-1), H^{\prime}-2\right),[H-3)$ ou $\left.\left.\left.H^{\prime}-3\right)\right\}\right\}$ avec une normalisation scalaire $\left(v_{n}\right)$ comme dans le théorème 2.5, alors:

$$
\frac{\left\langle K^{u, y}\right\rangle_{n}}{\log v_{n}^{2}} \underset{n \rightarrow \infty}{\longrightarrow}\left({ }^{*} u C u\right)\left({ }^{*} y C y\right) \text { p.s. }
$$

pour tous $u, y$ dans $\mathbb{R}^{d}$. [Si la matrice $C$ est inversible, ce résultat est également vrai sous les hypothèses $H$-1) et $\left.H^{\prime}-3\right)$ uniquement ]. 
3) Si $M$ satisfait les hypothèses $\{H-1), H$-2), $H^{\prime}$-3) et $H$-4) $\}$ avec une normalisation $\left(V_{n}\right)$ satisfaisant aux conditions de croissance exponentielle $\left(\mathcal{C}^{\prime}\right)$, alors :

$$
\frac{\left\langle H^{u}\right\rangle_{n}}{n} \underset{n \rightarrow \infty}{\longrightarrow}{ }^{*} u\left(C-\Lambda_{\infty} C{ }^{*} \Lambda_{\infty}\right) u\left(\Lambda_{\infty} C{ }^{*} \Lambda_{\infty}\right) \quad p . s .
$$

Preuve du lemme 5.3.

1) Il suffit de montrer que pour tout $x \in \mathbb{R}^{d}$, on a :

Or, posant comme dans le lemme précédent :

$$
\frac{{ }^{*} x\left\langle H^{u}\right\rangle_{n} x}{A_{n}} \underset{n \rightarrow \infty}{\longrightarrow}\left({ }^{*} u \widetilde{C} u\right)\left({ }^{*} x C x\right) \text { p.s. }
$$

$$
\begin{aligned}
& F_{n}=F_{n}(u)=\sum_{k=0}^{n} b_{k}^{-1} a_{k}^{-1 *} u V_{k+1}^{-1} \Delta\langle M\rangle_{k+1}{ }^{*} V_{k+1}^{-1} u, \\
& F_{0}=0, b_{k+1}^{-1}=\exp \left(A_{k+1}\right)-\exp \left(A_{k}\right),
\end{aligned}
$$

on vérifie que :

$$
{ }^{*} x\left\langle H^{u}\right\rangle_{n+1} x=a_{n} b_{n} \exp \left(A_{n}\right)\left\langle x, \Lambda_{n} Z_{n}\right\rangle^{2} \exp \left(-A_{n}\right) F_{n}+\sum_{k=1}^{n-1} a_{k+1}\left\langle x, \Lambda_{k+1} Z_{k+1}\right\rangle^{2} \exp \left(-A_{k}\right) F_{k}-G_{n}
$$

où

$$
G_{n}=\sum_{k=1}^{n-1}\left\{a_{k+1} b_{k+1} \exp \left(A_{k+1}\right)\left\langle x, \Lambda_{k+1} Z_{k+1}\right\rangle^{2}-a_{k} b_{k} \exp \left(A_{k}\right)\left\langle x, \Lambda_{k} Z_{k}\right\rangle^{2}\right\} \exp \left(-A_{k}\right) F_{k} .
$$

Grâce aux propriétés suivantes :

$\left\langle x, \Lambda_{k+1} Z_{k+1}\right\rangle^{2}=\left\langle{ }^{*} \Lambda_{k+1} x, \Lambda_{k} Z_{k}\right\rangle^{2}+\left\langle{ }^{*} \Lambda_{k+1} x, X_{k+1}\right\rangle^{2}+2\left\langle{ }^{*} \Lambda_{k+1} x, \Lambda_{k} Z_{k}\right\rangle\left\langle{ }^{*} \Lambda_{k+1} x, X_{k+1}\right\rangle ;$ $\left|a_{k+1} b_{k+1} \exp \left(A_{k+1}\right)-a_{k} b_{k} \exp \left(A_{k}\right)\right|=o\left(a_{k}\right),(k \rightarrow \infty) ;$

et

$\left|a_{k+1} b_{k+1} \exp \left(A_{k+1}\right)\left\langle{ }^{*} \Lambda_{k+1} x, \Lambda_{k} Z_{k}\right\rangle^{2}-a_{k} b_{k} \exp \left(A_{k}\right)\left\langle x, \Lambda_{k} Z_{k}\right\rangle^{2}\right|$

$\leq 2 a_{k+1} b_{k+1} \exp \left(A_{k+1}\right)\|x\|^{2}\left\|I-\Lambda_{k+1}\right\|\left\|Z_{k}\right\|^{2}+\left|a_{k+1} b_{k+1} \exp \left(A_{k+1}\right)-a_{k} b_{k} \exp \left(A_{k}\right)\right|\left\langle x, \Lambda_{k} Z_{k}\right\rangle^{2}$

$=O\left(a_{k}\left\|Z_{k}\right\|^{2}\right)$ p.s.

nous allons montrer que :

En effet :

$$
G_{n}=o\left(A_{n}\right) \text { p.s. }(n \rightarrow \infty) .
$$

$$
\begin{aligned}
G_{n}= & \left(a_{n} b_{n} \exp \left(A_{n}\right)\left\langle x, \Lambda_{n} Z_{n}\right\rangle^{2}-a_{1} b_{1} \exp \left(A_{1}\right)\left\langle x, \Lambda_{1} Z_{1}\right\rangle^{2}\right) * \widetilde{C} u \\
& +2 \sum_{k=1}^{n-1} a_{k+1} b_{k+1} \exp \left(A_{k+1}\right)\left\langle{ }^{*} \Lambda_{k+1} x, \Lambda_{k} Z_{k}\right\rangle \exp \left(-A_{k}\right) F_{k}\left\langle{ }^{*} \Lambda_{k+1} x, X_{k+1}\right\rangle \\
& -2{ }^{*} u \widetilde{C} u \sum_{k=1}^{n} a_{k+1} b_{k+1} \exp \left(A_{k+1}\right)\left\langle{ }^{*} \Lambda_{k+1} x, \Lambda_{k} Z_{k}\right\rangle\left\langle{ }^{*} \Lambda_{k+1} x, X_{k+1}\right\rangle \\
& +O\left(\sum_{k=1}^{n}\left\|X_{k+1}\right\|^{2}\left|\exp \left(-A_{k}\right) F_{k}-{ }^{*} u \widetilde{C} u\right|\right)+O\left(\sum_{k=1}^{n} a_{k}\left\|Z_{k}\right\|^{2}\left|\exp \left(-A_{k}\right) F_{k}-{ }^{*} u \widetilde{C} u\right|\right) .
\end{aligned}
$$

Le deuxième terme du membre de droite de cette égalité est une martingale scalaire dont la variation quadratique prévisible est, grâce à la propriété (5.8-bis), $o\left(A_{n}\right)$ p.s. Le troisième terme a également le même comportement 
asymptotique. Le résultat à établir découle alors de (5.2) du lemme précédent et de la LFQ qui est valable dans le cadre 1) du lemme.

2) Si les hypothèses H-1) et H'-3) sont satisfaites avec une suite $\left(v_{n}\right)$ croissant vers $\infty$ et telle que $\frac{v_{n}}{v_{n+1}} \underset{n \rightarrow \infty}{\longrightarrow} 1$ et si la matrice $C$ est inversible, alors on peut appliquer la loi forte quadratique à la martingale scalaire $\left(\left\langle u, M_{n}\right\rangle\right)$ avec la pondération aléatoire prévisible $\left({ }^{*} y\langle M\rangle_{k} y \mathbf{1}_{\left\{{ }^{*} y\langle M\rangle_{k} y \neq 0\right\}}\right)$, car ${ }^{*} y C y>0$ p.s. pour tout vecteur $y \in \mathbb{R}^{d}$ non nul. En effet dans ce cas, on a :

$$
\frac{{ }^{*} u\langle M\rangle_{n} u}{{ }^{*} y\langle M\rangle_{n} y} \underset{n \rightarrow \infty}{\longrightarrow} \frac{{ }^{*} u C u}{{ }^{*} y C y} \text { et }\left\langle u, M_{n}\right\rangle=o\left(\sqrt{{ }^{*} y\langle M\rangle_{n} y \log ^{*} y\langle M\rangle_{n} y}\right) \text { p.s. }
$$

(cf. $[4,6,14,25])$; et par conséquent :

$$
\left(\log { }^{*} y\langle M\rangle_{n+1} y\right)^{-1} \sum_{k=0}^{n} \frac{\left\langle u, M_{k}\right\rangle^{2}}{{ }^{*} y\langle M\rangle_{k} y}\left(1-\frac{{ }^{*} y\langle M\rangle_{k} y}{{ }^{*} y\langle M\rangle_{k+1} y}\right) \mathbf{1}_{\left\{{ }^{*} y\langle M\rangle_{k} y \neq 0\right\}} \underset{n \rightarrow \infty}{\longrightarrow} \frac{{ }^{*} u C u}{{ }^{*} y C y} \text { p.s. }
$$

Or, d'après (5.26) :

$\left\langle K^{u, y}\right\rangle_{n+1}=\sum_{k=0}^{n} \frac{\left\langle u, M_{k}\right\rangle^{2}}{{ }^{*} y\langle M\rangle_{k} y} \frac{{ }^{*} y\langle M\rangle_{k} y}{v_{k+1}^{2}} \frac{{ }^{*} y\langle M\rangle_{k+1} y}{v_{k+1}^{2}}\left(1-\frac{{ }^{*} y\langle M\rangle_{k} y}{{ }^{*} y\langle M\rangle_{k+1} y}\right) \mathbf{1}_{\left\{{ }^{*} y\langle M\rangle_{k} y \neq 0\right\}}$

donc :

$$
\left(\log v_{n+1}^{2}\right)^{-1}\left\langle K^{u, y}\right\rangle_{n+1} \underset{n \rightarrow \infty}{\longrightarrow}\left({ }^{*} u C u\right)\left({ }^{*} y C y\right) \quad \text { p.s. }
$$

et l'assertion entre crochets de la partie 2) du lemme est établie. Le début de cette partie est un cas particulier de la partie 1) du lemme, car la normalisation scalaire $\left(v_{n}\right)$ vérifie la condition suivante analogue à C-3-bis) :

$$
\frac{v_{n}}{v_{n+1}}=1-a_{n}\left(1+\frac{v_{n}}{v_{n+1}}\right)^{-1}=1-\frac{1}{2} a_{n}+o\left(a_{n}\right) \text { avec } a_{n}=1-\left(\frac{v_{n}}{v_{n+1}}\right)^{2} .
$$

3) D'après le théorème 3.1, si les hypothèses $\left.\{\mathrm{H}-1), \mathrm{H}-2), \mathrm{H}^{\prime}-3\right)$ et H-4)\} sont vérifiées avec une normalisation satisfaisant aux conditions $\left(\mathcal{C}^{\prime}\right)$, on a :

$$
n^{-1} \sum_{k=1}^{n} V_{k}^{-1} M_{k}{ }^{*} M_{k}{ }^{*} V_{k}^{-1} \underset{n \rightarrow \infty}{\longrightarrow} C \text { p.s. }
$$

donc sous ces hypothèses :

$\left\langle H^{u}\right\rangle_{n+1}=\Lambda_{\infty}\left(\sum_{k=0}^{n} V_{k}^{-1} M_{k}{ }^{*} M_{k}{ }^{*} V_{k}^{-1}\right){ }^{*} \Lambda_{\infty}{ }^{*} u\left(C-\Lambda_{\infty} C{ }^{*} \Lambda_{\infty}\right) u+o\left(\sum_{k=1}^{n}\left\|V_{k}^{-1} M_{k}\right\|^{2}\right) ;$

d'où :

Le lemme est établi.

$$
\frac{\left\langle H^{u}\right\rangle_{n}}{n} \underset{n \rightarrow \infty}{\longrightarrow}{ }^{*} u\left(C-\Lambda_{\infty} C{ }^{*} \Lambda_{\infty}\right) u\left(\Lambda_{\infty} C{ }^{*} \Lambda_{\infty}\right) .
$$

Grâce au lemme précédent, on obtient aisément le suivant :

\section{Lemme 5.3-bis.}

1) Dans le cadre des parties 1 ou 3 du lemme 5.3, les propriétés suivantes ont lieu pour la convergence p.s. :

$$
\frac{\left\langle H^{\prime}\right\rangle_{n}}{A_{n}} \underset{n \rightarrow \infty}{\longrightarrow} \sum_{j=1}^{d} \sum_{l=1}^{d}\left({ }^{*} e_{j} \widetilde{C} e_{l}\right)\left({ }^{*} x_{j} C x_{l}\right)
$$


ou

$$
\frac{\left\langle H^{\prime}\right\rangle_{n}}{n} \underset{n \rightarrow \infty}{\longrightarrow} \sum_{j=1}^{d} \sum_{l=1}^{d}\left({ }^{*} e_{j}\left(C-\Lambda_{\infty} C{ }^{*} \Lambda_{\infty}\right) e_{l}\right)\left({ }^{*} x_{j} \Lambda_{\infty} C{ }^{*} \Lambda_{\infty} x_{l}\right) .
$$

2) Et dans le cadre de la partie 2 du lemme 5.3, on a :

$$
\frac{\left\langle K^{\prime}\right\rangle_{n}}{v_{n}^{2}} \underset{n \rightarrow \infty}{\longrightarrow} \sum_{j=1}^{d} \sum_{l=1}^{d}\left({ }^{*} e_{j} C e_{l}\right)\left({ }^{*} u_{j} C u_{l}\right)
$$

c)Vérification de la condition de Lindeberg pour les martingales $\left(H_{n}^{\prime}\right),\left(K_{n}^{\prime}\right)$

Lemme 5.4. La martingale $\left(H_{n}^{\prime}\right)$ vérifie la condition de Lindeberg lorsqu'on se place dans le cadre de la partie 1 ou bien dans le cadre de la partie 3 du lemme 5.3. Cette condition est également vérifiée par la martingale $\left(K_{n}^{\prime}\right)$ dans le cadre de la partie 2 de ce lemme (c'est à dire sous les hypothèses \{H-1), H-2), [H-3 ou H'-3] et $H$-4\} ou bien $\left.\{H-1), H^{\prime}-2\right),\left[H-3\right.$ ou $\left.\left.H^{\prime}-3\right]\right\}$.

Preuve du lemme 5.4

Cas de la martingale $\left(H_{n}^{\prime}\right)$

Pour $\varepsilon>0$ et $u, w$ vecteurs de $\mathbb{R}^{d}$, posons :

avec :

$$
\Delta_{n+1}^{u, w}(\varepsilon)=B_{n+1}^{-1} \sum_{k=0}^{n} \mathbb{E}\left\{\left\|\Delta H_{k+1}^{u}\right\|^{2} \mathbf{1}_{\left\{\left\|\Delta H_{k+1}^{w}\right\|>\varepsilon \sqrt{B_{n+1}}\right\}} / \mathfrak{F}_{k}\right\}
$$

$B_{n+1}=A_{n+1}$ si on se place dans le cadre de la partie 1 du lemme 5.3 (cas 1$)$;

$B_{n+1}=n+1$ si on se place dans le cadre de la partie 3 de ce lemme (cas 3 ).

Pour que la martingale $\left(H_{n}^{\prime}\right)$ vérifie la condition de Lindeberg pour la convergence p.s., il suffit de montrer que dans le cas 1 ou 3 :

$$
\Delta_{n+1}^{u, w}(\varepsilon) \underset{n \rightarrow \infty}{\longrightarrow} 0 \quad \text { p.s. }
$$

Pour cela, on remarque que pour tout $A>0$ :

$$
\Delta_{n+1}^{u, w}(\varepsilon) \leq \Delta_{n+1}^{\prime}(\varepsilon, A)+\Delta^{\prime \prime}{ }_{n+1}(\varepsilon, A)
$$

avec :

$$
\begin{aligned}
\Delta_{n+1}^{\prime}(\varepsilon, A)= & B_{n+1}^{-1} \sum_{k=0}^{n}\left\|V_{k}^{-1} M_{k}\right\|^{2} \times \mathbf{1}_{\left\{\left\|V_{k}^{-1} M_{k}\right\| \leq A\right\}} \\
& \times \mathbb{E}\left\{\left\langle u, V_{k+1}^{-1} \Delta M_{k+1}\right\rangle^{2} \times \mathbf{1}_{\left\{\left\|V_{k}^{-1} M_{k}\right\|\left|\left\langle w, V_{k+1}^{-1} \Delta M_{k+1}\right\rangle\right|>\varepsilon \sqrt{B_{n+1}}\right\}} / \mathfrak{F}_{k}\right\}
\end{aligned}
$$

$\Delta{ }^{n+1}(\varepsilon, A)=B_{n+1}^{-1} \sum_{k=0}^{n}\left\|V_{k}^{-1} M_{k}\right\|^{2} \times \mathbf{1}_{\left\{\left\|V_{k}^{-1} M_{k}\right\|>A\right\}} \mathbb{E}\left\{\left\langle u, V_{k+1}^{-1} \Delta M_{k+1}\right\rangle^{2} / \mathfrak{F}_{k}\right\} ;$

et que dans le cas 1 et 3 , les deux propriétés suivantes ont lieu p.s. (cf. Ths. 2.1 et 3.1) :

$B_{n+1}^{-1} \sum_{k=0}^{n}\left\|V_{k}^{-1} M_{k}\right\|^{2} \mathbb{E}\left\{\left\langle u, V_{k+1}^{-1} \Delta M_{k+1}\right\rangle^{2} / \mathfrak{F}_{k}\right\} \underset{n \rightarrow \infty}{\longrightarrow} \begin{cases}{ }^{*} u \widetilde{C} u \operatorname{tr}\{C\} & \operatorname{cas} 1 \\ { }^{*} u\left(C-\Lambda_{\infty} C{ }^{*} \Lambda_{\infty}\right) u \operatorname{tr}\{C\} & \operatorname{cas} 3 .\end{cases}$

$$
\max _{0 \leq k \leq n} \frac{\left\|V_{k+1}^{-1} \Delta M_{k+1}\right\|}{\sqrt{B_{n+1}}} \underset{n \rightarrow \infty}{\longrightarrow} 0
$$


On en déduit que :

$$
\Delta_{n+1}^{\prime}(\varepsilon, A) \underset{n \rightarrow \infty}{\longrightarrow} 0 \text { p.s. }
$$

en exploitant la majoration suivante, valable pour tout $\delta>0$ :

$$
\begin{aligned}
& \left.\Delta_{n+1}^{\prime}(\varepsilon, A) \leq\left(\frac{A \delta}{\varepsilon}+\mathbf{1} \max _{0 \leq k \leq n}\left|\left\langle u, V_{k+1}^{-1} \Delta M_{k+1}\right\rangle\right|+\left|\left\langle w, V_{k+1}^{-1} \Delta M_{k+1}\right\rangle\right|>\delta \sqrt{B_{n+1}}\right\}\right) \\
& \times\left(B_{n+1}^{-1} \sum_{k=0}^{n}\left\|V_{k}^{-1} M_{k}\right\|^{2} \mathbb{E}\left\{\left\langle u, V_{k+1}^{-1} \Delta M_{k+1}\right\rangle^{2} / \mathfrak{F}_{k}\right\}\right) .
\end{aligned}
$$

Par ailleurs, d'après la loi forte logarithmique (cf. Cor. 2.2 et Rem. 3 de la Sect. 3.1) :

$$
\varlimsup_{n \rightarrow \infty} \Delta "_{n+1}(\varepsilon, A)=\operatorname{cte} \int\|x\|^{2} \mathbf{1}\{\|x\|>A\} d \mu_{\infty}(x),
$$

donc compte tenu de $(5.33,5.34)$ et $(5.35)$, p.s. :

$$
\lim _{A \rightarrow \infty} \varlimsup_{n \rightarrow \infty} \Delta{ }_{n+1}(\varepsilon, A) \leq \text { cte } \lim _{A \rightarrow \infty} \int\|x\|^{2} \mathbf{1}\{\|x\|>A\} d \mu_{\infty}(x)=0,
$$

puisque dans le cas 1 et $3: \int\|x\|^{2} d \mu_{\infty}(x)=\operatorname{tr}\{C\}<\infty$ p.s.

\section{Cas de la martingale $\left(K_{n}^{\prime}\right)$}

La condition de Lindeberg pour la martingale $\left(K_{n}^{\prime}\right)$ sera réalisée dès que pour tous $y, z$ de $\mathbb{R}^{d}$ on a :

$$
\Delta_{n+1}^{y, z}(\varepsilon)=\left(\log v_{n+1}^{2}\right)^{-1} \sum_{l=0}^{n} \mathbb{E}\left\{\left\|\Delta K_{l+1}^{y}\right\|^{2} \mathbf{1}_{\left\{\left\|\Delta K_{l+1}^{z}\right\|>\varepsilon \sqrt{\log v_{n+1}^{2}}\right\}} / \mathfrak{F}_{l}\right\} \underset{n \rightarrow \infty}{\longrightarrow} 0 \text { p.s. }
$$

Pour établir cette propriété, on peut procéder comme ci-dessus car le cas 2 est un exemple particulier du cas 1. Le lemme est établi.

d) Étude de la convergence en loi des suites $\left\{A_{n}^{-1 / 2} H_{n}^{\prime}, V_{n}^{-1} M_{n}\right\}$ et $\left\{\left(\log v_{n+1}^{2}\right)^{-1 / 2} K_{n}^{\prime}, \frac{M_{n}}{v_{n}}\right\}$.

Pour $y$ dans $\mathbb{R}^{d}$, posons :

$$
P_{n+1}=\left(\begin{array}{c}
K_{n+1}^{\prime} \\
\left\langle M_{n+1}, y\right\rangle
\end{array}\right)=\sum_{k=0}^{n} \sum_{j=1}^{d}\left(\begin{array}{c}
v_{k+1}^{-2}\left\langle u_{j}, M_{k}\right\rangle \\
\left\langle y, e_{j}\right\rangle
\end{array}\right)\left\langle\Delta M_{k+1}, e_{j}\right\rangle .
$$

La suite $\left(P_{n+1}\right)$ est une martingale, de variation quadratique prévisible :

avec :

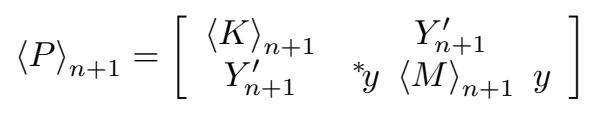

$$
Y_{n+1}^{\prime}=\sum_{k=0}^{n} \sum_{j=1}^{d} v_{k+1}^{-2}\left\langle u_{j}, M_{k}\right\rangle{ }^{*} e_{j}\left(\langle M\rangle_{k+1}-\langle M\rangle_{k}\right) e_{j}
$$


Tandis que, pour tous $n \geq 1$ et $y$ dans $\mathbb{R}^{d}$, la martingale :

$U_{p+1}^{(n+1)}=B_{n+1}^{-1 / 2} H_{p+1}^{\prime}+\left\langle y, V_{n+1}^{-1} M_{p+1}\right\rangle$

$$
=\sum_{k=0}^{p} \sum_{j=1}^{d}\left\langle\Delta M_{k+1}, B_{n+1}^{-1 / 2}\left\langle x_{j}, \Lambda_{k} V_{k}^{-1} M_{k}\right\rangle{ }^{*} V_{k+1}^{-1} e_{j}+\left\langle y, e_{j}\right\rangle{ }^{*} V_{n+1}^{-1} e_{j}\right\rangle
$$

avec $B_{n+1}=A_{n+1}$ ou $B_{n+1}=n+1$ est telle que :

$$
\left\langle U^{(n+1)}\right\rangle_{n+1}=B_{n+1}^{-1}\left\langle H^{\prime}\right\rangle_{n+1}+{ }^{*} y C_{n+1} y+2 W_{n+1}^{\prime} .
$$

où :

$$
W_{n+1}^{\prime}=B_{n+1}^{-1 / 2} \sum_{k=0}^{n} \sum_{j=1}^{d}\left\langle x_{j}, \Lambda_{k} V_{k}^{-1} M_{k}\right\rangle{ }^{*} e_{j} V_{k+1}^{-1}\left(\langle M\rangle_{k+1}-\langle M\rangle_{k}\right){ }^{*} V_{n+1}^{-1} y
$$

\section{Lemme 5.5.}

1) Dans le cadre de la partie 2 du lemme 5.3, on a :

$$
\frac{Y_{n+1}^{\prime}}{\sqrt{v_{n}^{2} \log v_{n}^{2}}} \underset{n \rightarrow \infty}{\longrightarrow} 0 \quad \text { p.s. ; }
$$

et sous les hypothèses $\{H-1), H^{\prime}$-2), $\left[H\right.$-3 ou $H^{\prime}$-3] $\}$ la suite $\left(\frac{K_{n+1}^{\prime}}{\sqrt{\log v_{n+1}^{2}}}, \frac{\left\langle M_{n+1}, y\right\rangle}{v_{n+1}}\right)$ vérifie la condition de Lindeberg, à savoir :

$$
\begin{aligned}
\widehat{\Delta}_{n+1}(\varepsilon)= & \sum_{l=0}^{n} \mathbb{E}\left\{\left(\left[\left(\log v_{n+1}^{2}\right)^{-1 / 2} \Delta K_{l+1}^{\prime}\right]^{2}+\left(v_{n+1}^{-1} \Delta\left\langle M_{l+1}, y\right\rangle\right)^{2}\right)\right. \\
& \left.\times \mathbf{1}\left\{\sqrt{\left(\log v_{n+1}^{2}\right)^{-1}\left(\Delta K_{l+1}^{\prime}\right)^{2}+v_{n+1}^{-2}\left(\Delta\left\langle M_{l+1}, y\right\rangle\right)^{2}} \geq \varepsilon\right\} / \mathfrak{F}_{l}\right\} \underset{n \rightarrow \infty}{\longrightarrow} 0 \quad \text { p.s., } \quad \forall \varepsilon>0 .
\end{aligned}
$$

2) Dans le cadre des parties 1 ou 3 du lemme 5.3, on a:

$$
W_{n+1}^{\sharp}=\left.\left.B_{n+1}^{-1 / 2} \sum_{k=0}^{n}\left|\left\langle x, \Lambda_{k} V_{k}^{-1} M_{k}\right\rangle\right|\right|^{*} u V_{k+1}^{-1}\left(\langle M\rangle_{k+1}-\langle M\rangle_{k}\right)^{*} V_{n+1}^{-1} y\right|_{n \rightarrow \infty} ^{\longrightarrow} 0 \text { p.s. }
$$

et donc:

$$
W_{n+1}^{\prime} \underset{n \rightarrow \infty}{\longrightarrow} 0 \text { p.s., pour tous } u, x, y \text { dans } \mathbb{R}^{d}
$$

Preuve du lemme 5.5.

1) Montrons la première assertion de la première partie du lemme. Pour cela, il suffit de montrer que :

où

$$
\frac{Y_{n}}{\sqrt{v_{n}^{2} \log v_{n}^{2}}} \underset{n \rightarrow \infty}{\longrightarrow} 0 \text { p.s. }
$$

$$
Y_{n+1}=\sum_{k=0}^{n} v_{k+1}^{-2}\left\langle u, M_{k}\right\rangle^{*} y\left(\langle M\rangle_{k+1}-\langle M\rangle_{k}\right) y
$$


$u, y$ sont deux vecteurs quelconques de $\mathbb{R}^{d}$. On a :

$$
\begin{aligned}
\frac{Y_{n+1}}{\sqrt{v_{n+1}^{2} \log v_{n+1}^{2}}} & =o\left(v_{n+1}^{-1} \sum_{k=0}^{n} \frac{{ }^{*} y\langle M\rangle_{k+1} y-{ }^{*} y\langle M\rangle_{k} y}{2 \sqrt{{ }^{*} y\langle M\rangle_{k+1} y}}\right) \\
\frac{Y_{n+1}}{\sqrt{v_{n+1}^{2} \log v_{n+1}^{2}}} & =o\left(v_{n+1}^{-1} \sum_{k=0}^{n} \int_{{ }^{*} y\langle M\rangle_{k} y}^{{ }^{*} y\langle M\rangle_{k+1} y} 2 x^{-1 / 2} d x\right)=o\left(\sqrt{\frac{{ }^{*} y\langle M\rangle_{n+1} y}{v_{n+1}^{2}}}\right) \\
& =o\left(\sqrt{{ }^{*} y C y}\right)=o(1) \text { p.s. }(n \rightarrow \infty)
\end{aligned}
$$

car, dans le cadre envisagé, on a :

$$
\max _{0 \leq k \leq n} \frac{\left|\left\langle u, M_{k}\right\rangle\right|}{v_{k}}=o\left(\sqrt{\log v_{n+1}^{2}}\right) \text { p.s. }(n \rightarrow \infty) .
$$

Lorsque l'hypothèse $\left.\mathrm{H}^{\prime}-2\right)$ a lieu, la vérification de la condition de Lindeberg pour le couple $\left(K_{n}^{\prime}, M_{n}\right)$ est immédiate compte tenu du lemme 5.4.

2) Dans le cadre de la partie 1 du lemme 5.3, la convergence p.s. de $\left(W_{n}^{\sharp}\right)$ vers 0 découle de la propriété :

$$
\max _{0 \leq k \leq n}\left\|V_{k}^{-1} M_{k}\right\|=o\left(A_{n+1}^{1 / 2}\right) \text { p.s. }(n \rightarrow \infty)
$$

valable dans le cadre envisagé et de l'inégalité suivante :

$$
\begin{aligned}
W_{n+1}^{\sharp} \leq & \|x\| A_{n}^{-1 / 2} \max _{0 \leq k \leq n}\left\|V_{k}^{-1} M_{k}\right\|\left(\sum_{k=0}^{n} \sqrt{{ }^{*} u V_{k+1}^{-1}\left(\langle M\rangle_{k+1}-\langle M\rangle_{k}\right)^{*} V_{k+1}^{-1} u}\right. \\
& \left.\times \sqrt{{ }^{*} y V_{n+1}^{-1}\left(\langle M\rangle_{k+1}-\langle M\rangle_{k}\right)^{*} V_{n+1}^{-1} y}\right) .
\end{aligned}
$$

Celle-ci implique que $W_{n+1}^{\sharp}=o(1)$ p.s. en vertu du lemme 5.2 et de la condition C-3) (qui a lieu sous C-3-bis)). En effet :

$$
\operatorname{tr}\left\{V_{n+1}^{-1}\left(\langle M\rangle_{k+1}-\langle M\rangle_{k}\right){ }^{*} V_{n+1}^{-1}\right\} \leq\left(\prod_{l=k+1}^{n}\left\|\Lambda_{l}\right\|^{2}\right) \operatorname{tr}\left\{V_{k+1}^{-1}\left(\langle M\rangle_{k+1}-\langle M\rangle_{k}\right){ }^{*} V_{k+1}^{-1}\right\}
$$

et d'après la condition C-3) : $1-\left\|\Lambda_{l}\right\|^{2} \leq \rho a_{l}(l \rightarrow \infty)$ pour un $\rho>0$, ce qui implique que pour $k$ assez grand :

Ainsi :

$$
\prod_{l=k+1}^{n}\left\|\Lambda_{l}\right\|^{2} \leq \exp \left\{-\rho \sum_{l=k+1}^{n} a_{l}\right\}=\exp \left\{-\rho\left(A_{n}-A_{k}\right)\right\} .
$$

$$
W_{n+1}^{\sharp} \leq \mathrm{c}^{t e} A_{n}^{-1 / 2} \max _{0 \leq k \leq n}\left\|V_{k}^{-1} M_{k}\right\| \sum_{k=0}^{n}\left[a_{k} \exp \left\{-\frac{1}{2} \rho\left(A_{n}-A_{k}\right)\right\} \operatorname{tr}\left\{\frac{1}{a_{k}} V_{k+1}^{-1}\left(\langle M\rangle_{k+1}-\langle M\rangle_{k}\right)^{*} V_{k+1}^{-1}\right\}\right]
$$

et d'après le lemme 5.2 la somme figurant au second membre de cette inégalité est bornée au sens de la convergence p.s., car :

$$
\exp \left(\frac{1}{2} \rho A_{k}\right)-\exp \left(\frac{1}{2} \rho A_{k-1}\right) \sim \frac{\rho}{2} a_{k} \exp \left(\frac{1}{2} \rho A_{k}\right),(k \rightarrow \infty)
$$


Dans le cadre de la partie 3 du lemme 5.3 , on a :

$$
\max _{0 \leq k \leq n}\left\|V_{k}^{-1} M_{k}\right\|=o(\sqrt{n}) \quad \text { p.s. ; }
$$

donc :

$$
W_{n+1}^{\sharp}=o\left(\sum_{k=0}^{n}\left\|V_{n+1}^{-1} V_{k+1}\right\|\right)=o(1) \quad \text { p.s. },
$$

car :

$$
\sum_{k=0}^{n}\left\|V_{n+1}^{-1} V_{k+1}\right\| \leq \sum_{k=0}^{n}\left\|\Lambda_{k+1}\right\| \ldots\left\|\Lambda_{n}\right\| \leq \operatorname{cte}+\frac{1}{1-r}
$$

pour tout $r$ tel que : $1>r \geq\left\|\Lambda_{\infty}\right\|=\lim _{n \rightarrow \infty}\left\|\Lambda_{n}\right\|$.

\section{Lemme 5.6.}

1) Dans le cadre des parties 1 ou bien 3 du lemme 5.3, on a:

avec :

$$
\prod_{k=0}^{n} \mathbb{E}\left\{\exp \left(i \Delta U_{k+1}^{(n+1)}\right) / \mathfrak{F}_{k}\right\} \underset{n \rightarrow \infty}{\longrightarrow} \Phi_{\infty}(\eta, y) \Psi_{\infty}\left(x_{1}, \ldots, x_{d}\right) \quad \text { p.s. }
$$

$\Psi_{\infty}\left(x_{1}, \ldots, x_{d}\right)=\exp \left\{-\frac{1}{2} \sum_{j=1}^{d} \sum_{l=1}^{d} * e_{j} \widetilde{C} e_{l}{ }^{*} x_{j} \Lambda_{\infty} C \Lambda_{\infty} x_{l}\right\}$

ou bien

$$
\Psi_{\infty}\left(x_{1}, \ldots, x_{d}\right)=\exp \left\{-\frac{1}{2} \sum_{j=1}^{d} \sum_{l=1}^{d}{ }^{*} e_{j}\left(C-\Lambda_{\infty} C{ }^{*} \Lambda_{\infty}\right) e_{l}{ }^{*} x_{j} C x_{l}\right\} .
$$

2) Dans le cadre de la partie 2 du lemme 5.3, on a:

$$
\prod_{k=0}^{n} \mathbb{E}\left\{\exp \left(i \Delta P_{k+1}\right) / \mathfrak{F}_{k}\right\} \underset{n \rightarrow \infty}{\longrightarrow} \Phi_{\infty}(\eta, y) \Psi_{\infty}\left(u_{1}, \ldots, u_{d}\right) \quad p . s .
$$

avec :

$$
\Psi_{\infty}\left(u_{1}, \ldots, u_{d}\right)=\exp \left\{-\frac{1}{2} \sum_{j=1}^{d} \sum_{l=1}^{d} * e_{j} C e_{l} * u_{j} C u_{l}\right\} .
$$

Preuve du lemme 5.6.

1) Posant :

$$
\begin{aligned}
& \Theta_{n+1}\left(x_{1}, \ldots, x_{d}, y\right)=\prod_{k=0}^{n} \mathbb{E}\left\{\exp \left(i \Delta U_{k+1}^{(n+1)}\right) / \mathfrak{F}_{k}\right\} \\
& \Phi_{n+1}(y)=\prod_{k=0}^{n} \mathbb{E}\left\{\exp \left(i\left\langle y, V_{n+1}^{-1} \Delta M_{k+1}\right\rangle\right) / \mathfrak{F}_{k}\right\} \\
& \Psi_{n+1}\left(x_{1}, \ldots, x_{d}\right)=\prod_{k=0}^{n} \mathbb{E}\left\{\exp \left(i B_{n+1}^{-1 / 2} \Delta H_{k+1}^{\prime}\right) / \mathfrak{F}_{k}\right\}
\end{aligned}
$$

on a :

$$
\begin{aligned}
& \mid \Theta_{n+1}\left(x_{1}, \ldots, x_{d}, y\right)-\Phi_{n+1}(y) \Psi_{n+1}(\left.x_{1}, \ldots, x_{d}\right) \mid \leq \frac{1}{\sqrt{B_{n+1}}} \sum_{k=0}^{n} \mathbb{E}\left\{\left|\left\langle y, V_{n+1}^{-1} \Delta M_{k+1}\right\rangle\right| \cdot\left|\Delta H_{k+1}^{\prime}\right| / \mathfrak{F}_{k}\right\} \\
&+\frac{1}{4 B_{n+1}} \sum_{k=0}^{n} \mathbb{E}\left\{\left\langle y, V_{n+1}^{-1} \Delta M_{k+1}\right\rangle^{2} / \mathfrak{F}_{k}\right\} \mathbb{E}\left\{\left(\Delta H_{k+1}^{\prime}\right)^{2} / \mathfrak{F}_{k}\right\}
\end{aligned}
$$


En effet, grâce à l'inégalité :

$$
\left|\prod_{k=0}^{n} a_{k}-\prod_{k=0}^{n} b_{k}\right| \leq \sum_{k=0}^{n}\left|b_{k}-a_{k}\right|
$$

valable si $\left|a_{k}\right| \leq 1$ et $\left|b_{k}\right| \leq 1$, le membre de gauche de (5.36) est majoré par :

$$
\begin{aligned}
& \sum_{k=0}^{n} \mid \mathbb{E}\left\{\left[1-\exp \left(i\left\langle y, V_{n+1}^{-1} \Delta M_{k+1}\right\rangle\right)\right]\left[1-\exp \left(i B_{n+1}^{-1 / 2} \Delta H_{k+1}^{\prime}\right)\right] / \mathfrak{F}_{k}\right\} \\
&-\mathbb{E}\left\{\left[1-\exp \left(i\left\langle y, V_{n+1}^{-1} \Delta M_{k+1}\right\rangle\right)\right] / \mathfrak{F}_{k}\right\} \times \mathbb{E}\left\{\left[1-\exp \left(i B_{n+1}^{-1 / 2} \Delta H_{k+1}^{\prime}\right)\right] / \mathfrak{F}_{k}\right\} \mid
\end{aligned}
$$

et cette quantité est majorée par le membre de droite de (5.36), compte tenu des inégalités classiques :

$$
\left|e^{i x}-1\right| \leq|x| ; \quad\left|e^{i x}-1-i x\right| \leq \frac{x^{2}}{2}
$$

et du fait que $\mathbb{E}\left\{V_{n+1}^{-1} \Delta M_{k+1} / \mathfrak{F}_{k}\right\}=\mathbb{E}\left\{\Delta H_{k+1}^{\prime} / \mathfrak{F}_{k}\right\}=0$.

Désormais désignons par $R_{n}$ le membre de gauche de (5.36).

2) Dans le cadre de la partie 1 du lemme 5.3, on a :

$$
R_{n} \underset{n \rightarrow \infty}{\longrightarrow} 0 \text { p.s. }
$$

En effet, d'après (5.36) et la première partie de la preuve précédente, on a :

$$
\begin{array}{r}
R_{n}=O\left(A_{n}^{-1 / 2} \max _{0 \leq k \leq n}\left\|V_{k}^{-1} M_{k}\right\| \sum_{k=0}^{n} \exp \left\{-\frac{1}{2} \rho\left(A_{n}-A_{k}\right)\right\} \operatorname{tr}\left\{V_{k+1}^{-1}\left(\langle M\rangle_{k+1}-\langle M\rangle_{k}\right){ }^{*} V_{k+1}^{-1}\right\}\right) \\
+O\left(A_{n+1}^{-1} \max _{0 \leq k \leq n} \mathbb{E}\left\{\left(\Delta H_{k+1}^{\prime}\right)^{2} / \mathfrak{F}_{k}\right\}\right) .
\end{array}
$$

Or, on sait que le premier terme du membre de droite de (5.38) tend vers 0 p.s. Et on sait également que la suite $\left(A_{n+1}^{-1}\left\langle H^{\prime}\right\rangle_{n+1}\right)$ est p.s. convergente ; donc le second terme du membre de droite de (5.38) tend p.s. vers 0 . La propriété (5.37) est établie.

3) Dans le cadre de la partie 3 du lemme 5.3, on a aussi (5.37). En effet :

$$
\begin{aligned}
R_{n} \leq & \frac{1}{\sqrt{n+1}} \sum_{k=0}^{n} \sqrt{\mathbb{E}\left\{\left\langle y, V_{n+1}^{-1} \Delta M_{k+1}\right\rangle^{2} / \mathfrak{F}_{k}\right\} \mathbb{E}\left\{\left(\Delta H_{k+1}^{\prime}\right)^{2} / \mathfrak{F}_{k}\right\}} \\
& +\frac{1}{4(n+1)} \sum_{k=0}^{n} \mathbb{E}\left\{\left\langle y, V_{n+1}^{-1} \Delta M_{k+1}\right\rangle^{2} / \mathfrak{F}_{k}\right\} \mathbb{E}\left\{\left(\Delta H_{k+1}^{\prime}\right)^{2} / \mathfrak{F}_{k}\right\} \\
R_{n} \leq & {\left[(n+1)^{-1} \max _{0 \leq k \leq n} \mathbb{E}\left\{\left(\Delta H_{k+1}^{\prime}\right)^{2} / \mathfrak{F}_{k}\right\}\right]^{1 / 2} \sum_{k=0}^{n}\left[{ }^{*} y V_{n+1}^{-1} \Delta\langle M\rangle_{k+1}{ }^{*} V_{n+1}^{-1} y\right]^{1 / 2} } \\
& +\frac{1}{4}(n+1)^{-1} \max _{0 \leq k \leq n} \mathbb{E}\left\{\left(\Delta H_{k+1}^{\prime}\right)^{2} / \mathfrak{F}_{k}\right\} \sum_{k=0}^{n}{ }^{*} y V_{n+1}^{-1} \Delta\langle M\rangle_{k+1}{ }^{*} V_{n+1}^{-1} y .
\end{aligned}
$$

Et d'après le lemme 5.3-bis, la suite $\left(n^{-1}\left\langle H^{\prime}\right\rangle_{n}\right)$ est p.s. convergente, donc :

Vu que pour tout $\alpha>0$

$$
(n+1)^{-1} \max _{0 \leq k \leq n} \mathbb{E}\left\{\left(\Delta H_{k+1}^{\prime}\right)^{2} / \mathfrak{F}_{k}\right\} \underset{n \rightarrow \infty}{\longrightarrow} 0 \text { p.s. }
$$

$$
\sum_{k=0}^{n}\left[{ }^{*} y V_{n+1}^{-1} \Delta\langle M\rangle_{k+1}{ }^{*} V_{n+1}^{-1} y\right]^{\alpha}=O\left(\sum_{k=0}^{n}\left\|V_{n+1}^{-1} V_{k}\right\|^{2 \alpha}\right)=O(1) \text { p.s. }
$$


la convergence p.s. vers 0 de la suite $\left(R_{n}\right)$ est établie.

4) Dans le cadre de la partie 2 du lemme 5.3, posant :

$\Theta_{n+1}\left(u_{1}, \ldots, u_{d}, y\right)=\prod_{l=0}^{n} \mathbb{E}\left\{\exp \left(i \Delta P_{l+1}\right) / \mathfrak{F}_{l}\right\}$,
$\Psi_{n+1}\left(u_{1}, \ldots, u_{d}\right)=\prod_{l=0}^{n} \mathbb{E}\left\{\exp \left(i\left(\log v_{n+1}^{2}\right)^{-1 / 2} \quad \Delta K_{l+1}^{\prime}\right) / \mathfrak{F}_{l}\right\}$.

L'inégalité (5.36) s'écrit :

$$
\begin{aligned}
R_{n} \leq & \left(\log v_{n+1}^{2}\right)^{-1 / 2} \sum_{l=0}^{n} \mathbb{E}\left\{\left|\frac{\left\langle y, \Delta M_{l+1}\right\rangle}{v_{n+1}}\right|\left|\Delta K_{l+1}^{\prime}\right| / \mathfrak{F}_{l}\right\} \\
& +\frac{1}{4}\left(\log v_{n+1}^{2}\right)^{-1} \sum_{l=0}^{n} \mathbb{E}\left\{\frac{\left\langle y, \Delta M_{l+1}\right\rangle^{2}}{v_{n+1}^{2}} / \mathfrak{F}_{l}\right\} \mathbb{E}\left\{\left(\Delta K_{l+1}^{\prime}\right)^{2} / \mathfrak{F}_{l}\right\} \\
& =O\left(\left(\log v_{n+1}^{2}\right)^{-1 / 2} \max _{0 \leq k \leq n}\left\|v_{k}^{-1} M_{k}\right\|\left\{v_{n+1}^{-1} \sum_{k=0}^{n} \operatorname{tr}\left\{v_{k+1}^{-1}\left(\langle M\rangle_{k+1}-\langle M\rangle_{k}\right)\right\}\right\}\right) \\
& +O\left(\left(\log v_{n+1}^{2}\right)^{-1} \max _{0 \leq k \leq n} \mathbb{E}\left\{\left(\Delta K_{k+1}^{\prime}\right)^{2} / \mathfrak{F}_{k}\right\}\right) .
\end{aligned}
$$

On en déduit (5.37), compte tenu des trois propriétés suivantes :

$$
\begin{gathered}
v_{n+1}^{-1} \sum_{k=0}^{n} \operatorname{tr}\left\{v_{k+1}^{-1}\left(\langle M\rangle_{k+1}-\langle M\rangle_{k}\right)\right\}=O\left(\frac{\sqrt{\operatorname{tr}\left(\langle M\rangle_{n+1}\right)}}{v_{n+1}}\right)=O(1) \text { p.s. ; } \\
\left(\log v_{n+1}^{2}\right)^{-1 / 2} \max _{0 \leq k \leq n}\left\|v_{k}^{-1} M_{k}\right\|_{n \rightarrow \infty}^{\longrightarrow} 0 \text { p.s. ; }
\end{gathered}
$$

et

$$
\left(\log v_{n+1}^{2}\right)^{-1} \max _{0 \leq k \leq n} \mathbb{E}\left\{\left(\Delta K_{k+1}^{\prime}\right)^{2} / \mathfrak{F}_{k}\right\} \underset{n \rightarrow \infty}{\longrightarrow} 0 \text { p.s. }
$$

Par hypothèse : $\Phi_{n+1}(y) \underset{n \rightarrow \infty}{\longrightarrow} \Phi_{\infty}(\eta, y)$ p.s. et d'après les lemmes 5.3-bis et 5.4 , on a :

$$
\Psi_{n+1}\left(x_{1}, \ldots, x_{d}\right) \underset{n \rightarrow \infty}{\longrightarrow} \Psi_{\infty}\left(x_{1}, \ldots, x_{d}\right) \text { p.s. ; }
$$

d'où le lemme.

Lemme 5.7. On se place dans le cadre de la partie 3) du lemme 5.3, en ajoutant les hypothèses H-5) et H-6) (cf. Sect. 3.1). Alors les martingales matricielles :

$$
H_{n+1}=\sum_{k=0}^{n}\left(V_{k+1}^{-1} M_{k}\right){ }^{*} X_{k+1}, \bar{H}_{n+1}=\sum_{k=0}^{n}\left(X_{k+1}{ }^{*} X_{k+1}-\mathbb{E}\left\{X_{k+1}{ }^{*} X_{k+1} / \mathfrak{F}_{k}\right\}\right),
$$

vérifient la propriété suivante :

$$
\left\{\frac{1}{\sqrt{n+1}} H_{n+1}, \frac{1}{\sqrt{n+1}} \bar{H}_{n+1}, V_{n}^{-1} M_{n}\right\} \underset{n \rightarrow \infty}{\stackrel{\mathcal{L}}{\longrightarrow}}\left\{\sum^{\prime}(C), \Sigma^{\prime \prime}(C), \sum(\eta)\right\}
$$

où, conditionnellement à $C=Y,\left(\begin{array}{c}\sum^{\prime}(Y) \\ \sum^{\prime \prime}(Y)\end{array}\right)$ est une v.a. gaussienne matricielle qu'on peut choisir indépendante du triplet $\left\{\eta, C,\left(\sum(x) ; x \in \mathfrak{X}\right)\right\}$ et dont la structure de covariance $\triangle(Y)$ est donnée par la formule (3.2). 
Preuve du lemme 5.7. On pose :

$\mathcal{H}_{n}=\operatorname{Vect}\left(H_{n}\right)=\left(H_{n}^{j} ; 1 \leq j \leq d\right), \overline{\mathcal{H}}_{n}=\operatorname{Vect}\left(\bar{H}_{n}\right)=\operatorname{Vect}\left(\bar{H}_{n}^{j} ; 1 \leq j \leq d\right)$ et $\mathbb{H}_{n}=\left(\begin{array}{c}\mathcal{H}_{n} \\ \overline{\mathcal{H}}_{n}\end{array}\right)$ où $\bar{H}_{n}^{j}=\bar{H}_{n} e_{j}\left(\right.$ et $\left.H_{n}^{j}=H_{n} e_{j}\right), 1 \leq j \leq d$.

Alors la variation quadratique prévisible de la martingale $\mathbb{H}$ s'écrit :

avec :

$$
\langle\mathbb{H}\rangle_{n+1}=\left(\begin{array}{ll}
\langle\mathcal{H}\rangle_{n+1} & \langle\mathcal{H}, \overline{\mathcal{H}}\rangle_{n+1} \\
*\langle\mathcal{H}, \overline{\mathcal{H}}\rangle_{n+1} & \langle\overline{\mathcal{H}}\rangle_{n+1}
\end{array}\right)
$$

- $\langle\mathcal{H}\rangle_{n+1}=\left(\left\langle H^{i}, H^{j}\right\rangle_{n+1}\right)_{1 \leq i, j \leq d}$ et

$$
\left\langle H^{i}, H^{j}\right\rangle_{n+1}=\sum_{k=0}^{n}\left(V_{k+1}^{-1} M_{k}\right) *\left(V_{k+1}^{-1} M_{k}\right) * e_{i} \mathbb{E}\left\{X_{k+1}{ }^{*} X_{k+1} / \mathfrak{F}_{k}\right\} e_{j}
$$

- $\langle\overline{\mathcal{H}}\rangle_{n+1}=\left(\left\langle\bar{H}^{i}, \bar{H}^{j}\right\rangle_{n+1}\right)_{1<i, j \leq d}$ et $\left\langle\bar{H}^{i}, \bar{H}^{j}\right\rangle_{n+1}=n \mathcal{L}_{n}(i, j)$;

- $\langle\mathcal{H}, \overline{\mathcal{H}}\rangle_{n+1}=\left(\left\langle H^{i}, \bar{H}^{j}\right\rangle_{n+1}\right)_{1 \leq i, j \leq d}$ et

$$
\left\langle H^{i}, \bar{H}^{j}\right\rangle_{n+1}=\sum_{k=0}^{n}\left(V_{k+1}^{-1} M_{k}\right) \mathbb{E}\left\{{ }^{*} e_{i}\left(X_{k+1}{ }^{*} X_{k+1}\right) e_{j}{ }^{*} X_{k+1} / \mathfrak{F}_{k}\right\}=n \mathcal{J}_{n}(i, j) .
$$

1) Étude du comportement asymptotique de la variation quadratique prévisible de $\mathbb{H}$ Compte tenu du lemme 5.3-bis et des hypothèses, on a :

$\frac{1}{n+1}\left\langle H^{i}, H^{j}\right\rangle_{n+1} \underset{n \rightarrow \infty}{\longrightarrow}\left({ }^{*} e_{i}\left(C-\Lambda_{\infty} C^{*} \Lambda_{\infty}\right) e_{j}\right)\left(\Lambda_{\infty} C^{*} \Lambda_{\infty}\right)$ p.s. ;

$\frac{1}{n+1}\left\langle\bar{H}^{i}, \bar{H}^{j}\right\rangle_{n+1} \underset{n \rightarrow \infty}{\longrightarrow} \mathcal{L}(i, j)$ p.s. ;

$\frac{1}{n+1}\left\langle H^{i}, \bar{H}^{j}\right\rangle_{n+1} \underset{n \rightarrow \infty}{\longrightarrow} \mathcal{J}(i, j)$ p.s.

Ainsi :

$$
\frac{1}{n}\langle\mathbb{H}\rangle_{n} \underset{n \rightarrow \infty}{\longrightarrow} \triangle(C) \text { p.s. }
$$

où $\triangle(C)$ est la matrice définie par (3.1).

2) Vérification de la condition de Lindeberg pour la martingale $\mathbb{H}$

Pour tout $\varepsilon>0$, on a :

$$
\frac{1}{n} \sum_{k=0}^{n} \mathbb{E}\left\{\left\|\Delta \mathbb{H}_{k+1}\right\|^{2} 1_{\left\{\left\|\mathbb{H}_{k+1}\right\|>\varepsilon \sqrt{n}\right\}} / \mathfrak{F}_{k}\right\} \underset{n \rightarrow \infty}{\longrightarrow} 0 \text { p.s. }
$$

En effet :

$$
\begin{aligned}
& \frac{1}{n} \sum_{k=0}^{n} \mathbb{E}\left\{\left\|\Delta \mathbb{H}_{k+1}\right\|^{2} 1_{\left\{\left\|\mathbb{H}_{k+1}\right\|>\varepsilon \sqrt{n}\right\}} / \mathfrak{F}_{k}\right\} \leq \frac{1}{n} \sum_{k=0}^{n} \mathbb{E}\left\{\left\|\Delta \mathcal{H}_{k+1}\right\|^{2} 1_{\left\{\left\|\mathcal{H}_{k+1}\right\|+\left\|\overline{\mathcal{H}}_{k+1}\right\|>\varepsilon \sqrt{n}\right\}} / \mathfrak{F}_{k}\right\} \\
& +\frac{1}{n} \sum_{k=0}^{n} \mathbb{E}\left\{\left\|\Delta \overline{\mathcal{H}}_{k+1}\right\|^{2} 1_{\left\{\left\|\mathcal{H}_{k+1}\right\|+\left\|\overline{\mathcal{H}}_{k+1}\right\|>\varepsilon \sqrt{n}\right\}} / \mathfrak{F}_{k}\right\} \\
& =o\left(\frac{1}{n} \sum_{k=0}^{n}\left\|V_{k+1}^{-1} M_{k}\right\|^{2}\right)+o\left(\frac{1}{n} \sum_{k=0}^{n} \mathbb{E}\left\{\left(\left\|X_{k+1}\right\|^{2}-\mathbb{E}\left\{\left\|X_{k+1}\right\|^{2} / \mathfrak{F}_{k}\right\}\right)^{2} / \mathfrak{F}_{k}\right\}\right) \\
& =o(1) \text { p.s., }
\end{aligned}
$$

car les propriétés suivantes résultant de l'hypothèse $\mathrm{H}-5)$ :

$$
\begin{aligned}
& \left\|X_{k+1}\right\|^{2}=o(\sqrt{k}) \text { et } \\
& \left\|V_{k+1}^{-1} M_{k}\right\|\left\|X_{k+1}\right\|=O\left(\left\|X_{k+1}\right\| \sum_{l=0}^{k} V_{k}^{-1} V_{l}\left\|X_{l}\right\|\right)=O\left(\left\|X_{k+1}\right\|^{2}\right) \text { p.s., }
\end{aligned}
$$


impliquent que :

$1_{\left\{\left\|\mathcal{H}_{k+1}\right\|+\left\|\overline{\mathcal{H}}_{k+1}\right\|>\varepsilon \sqrt{n}\right\}} \leq 1_{\left\{\left|\left\|X_{k+1}\right\|^{2}-\mathbb{E}\left\{\left\|X_{k+1}\right\|^{2} / \mathfrak{F}_{k}\right\}\right|>\frac{\varepsilon}{2} \sqrt{n}\right\}}+1_{\left\{\left\|V_{k+1}^{-1} M_{k}\right\|\left\|X_{k+1}\right\|>\frac{\varepsilon}{2} \sqrt{n}\right\}}$ $\leq 1_{\left\{k^{-1}\left(\left\|X_{k+1}\right\|^{2}-\mathbb{E}\left\{\left\|X_{k+1}\right\|^{2} / \mathfrak{F}_{k}\right\}\right)^{2}>\frac{\varepsilon^{2}}{4}\right\}}+1_{\left\{k^{-1 / 2}\left\|V_{k+1}^{-1} M_{k}\right\|\left\|X_{k+1}\right\|>\frac{\varepsilon}{2}\right\}}=o(1)$ p.s.

Comme les v.a. $\left(\mathbb{E}\left\{\left\|X_{n+1}\right\|^{2} / \mathfrak{F}_{n}\right\}\right),\left(\frac{1}{n} \sum_{k=0}^{n}\left\|V_{k+1}^{-1} M_{k}\right\|^{2}\right)$ et $\left(\frac{1}{n} \sum_{k=0}^{n} \mathbb{E}\left\{\left(\left\|X_{k+1}\right\|^{2}-\mathbb{E}\left\{\left\|X_{k+1}\right\|^{2} / \mathfrak{F}_{k}\right\}\right)^{2} /\right.\right.$ $\left.\mathfrak{F}_{k}\right\}$ ) sont presque-sûrement bornées, la condition de Lindeberg est vérifiée par $\mathbb{H}$.

3) Étude de la convergence en loi de la suite $\left\{\frac{1}{\sqrt{n}} \mathbb{H}_{n}, V_{n}^{-1} M_{n}\right\}$

Pour $x_{1}, \ldots, x_{d}, y, z_{1}, \ldots, z_{d}$ dans $\mathbb{R}^{d}$, posons :

$\mathbb{H}_{n+1}^{\prime}=\sum_{k=0}^{n}\left(\sum_{j=1}^{d}\left[{ }^{*} x_{j}\left(V_{k+1}^{-1} M_{k}\right){ }^{*} X_{k+1} e_{j}+{ }^{*} z_{j}\left(X_{k+1}{ }^{*} X_{k+1}-\mathbb{E}\left\{X_{k+1}{ }^{*} X_{k+1} / \mathfrak{F}_{k}\right\}\right) e_{j}\right]\right)$;

$U_{k+1}^{(n+1)}=\frac{1}{\sqrt{n+1}} \Delta \mathbb{H}_{k+1}^{\prime}+\left\langle y, V_{n+1}^{-1} \Delta M_{k+1}\right\rangle$;

$\Theta_{n+1}\left(x_{1}, \ldots, x_{d}, z_{1}, \ldots, z_{d}, y\right)=\prod_{k=0}^{n} \mathbb{E}\left\{\exp \left(i \Delta U_{k+1}^{(n+1)}\right) / \mathfrak{F}_{k}\right\}$

$\Phi_{n+1}(y)=\prod_{k=0}^{n} \mathbb{E}\left\{\exp \left(i\left\langle y, V_{n+1}^{-1} \Delta M_{k+1}\right\rangle\right) / \mathfrak{F}_{k}\right\}$

$\Psi_{n+1}\left(x_{1}, \ldots, x_{d}, z_{1}, \ldots, z_{d}\right)=\prod_{k=0}^{n} \mathbb{E}\left\{\exp \left(i \frac{1}{\sqrt{n+1}} \Delta \mathbb{H}_{k+1}^{\prime}\right) / \mathfrak{F}_{k}\right\}$

En procédant comme dans la preuve de la partie $1 \mathrm{du}$ lemme 5.6, on montre que :

$$
\left|\Theta_{n+1}\left(x_{1}, \ldots, x_{d}, z_{1}, \ldots, z_{d}, y\right)-\Phi_{n+1}(y) \Psi_{n+1}\left(x_{1}, \ldots, x_{d}, z_{1}, \ldots, z_{d}\right)\right| \underset{n \rightarrow \infty}{\longrightarrow} 0 \text { p.s. }
$$

On peut donc conclure que :

$$
\left\{\frac{1}{\sqrt{n}} \mathbb{H}_{n}, V_{n}^{-1} M_{n}\right\} \underset{n \rightarrow \infty}{\stackrel{\mathcal{L}}{\longrightarrow}}\left\{\left(\begin{array}{c}
\sum^{\prime}(C) \\
\sum^{\prime \prime}(C)
\end{array}\right), \sum(\eta)\right\}
$$

où, conditionnellement à $\{C=Y\},\left(\begin{array}{c}\sum^{\prime}(Y) \\ \sum^{\prime \prime}(Y)\end{array}\right)$ est une v.a. gaussienne matricielle indépendante du triplet $\left\{\eta, C,\left(\sum(x) ; x \in \mathfrak{X}\right)\right\}$ et dont la structure de covariance est donnée par la matrice $\triangle(Y)(c f .(3.2))$.

\section{Lemme 5.8.}

1) Soit $M$ une martingale vérifiant l'hypothèse $H$ "-3) avec une normalisation scalaire $\left(v_{n}\right)$ comme dans le théorème 2.5, alors :

i) $\left(\log v_{n}^{2}\right)^{-1 / 2} v_{n}^{-2}\left([M]_{n}-\langle M\rangle_{n}\right) \underset{n \rightarrow \infty}{\longrightarrow} 0$ et

$$
\left(\log v_{n+1}^{2}\right)^{-1 / 2} \sum_{k=0}^{n}\left\{1-\left(\frac{v_{k}}{v_{k+1}}\right)^{2}\right\} v_{k}^{-2}\left([M]_{k}-\langle M\rangle_{k}\right) \underset{n \rightarrow \infty}{\longrightarrow} 0 \text { p.s. }
$$

ii) $S i$, en outre, l'hypothèse $H$-1) est vérifiée et la série

$$
\sum_{n \geq 0}\left(\log v_{n+1}^{2}\right)^{-1 / 2} v_{n+1}^{-2}\left(\mathbb{E}\left\{\Delta M_{n+1}{ }^{*} \Delta M_{n+1} / \mathfrak{F}_{n}\right\}-\left(v_{n+1}^{2}-v_{n}^{2}\right) C\right)
$$


est p.s. convergente, alors :

$$
\begin{gathered}
\left(\log v_{n}^{2}\right)^{-1 / 2} v_{n}^{-2}[M]_{n} \underset{n \rightarrow \infty}{\longrightarrow} 0 \text { et } \\
\left(\log v_{n+1}^{2}\right)^{-1 / 2} \sum_{k=0}^{n}\left\{1-\left(\frac{v_{k}}{v_{k+1}}\right)^{2}\right\}\left\{v_{k}^{-2}\left([M]_{k}-C\right)\right\} \underset{n \rightarrow \infty}{\longrightarrow} 0 \text { p.s. }
\end{gathered}
$$

La seconde propriété de ii) est également vraie si $v_{n}^{-2}\langle M\rangle_{n}-C=O\left[\left(\log v_{n+1}^{2}\right)^{-{ }^{\rho}}\right]$ p.s. ou $\mathbb{E}\left(\left\|v_{n}^{-2}\langle M\rangle_{n}-C\right\|\right)$ $=O\left[\left(\log v_{n+1}^{2}\right)^{-^{\rho}}\right]$ pour un $\rho>\frac{1}{2}$.

2) Si $M$ vérifie l'hypothèse $H^{\prime \prime}$-3) avec une normalisation $\left(V_{n}\right)$ satisfaisant les conditions de croissance régulière $\mathrm{C}-1), \mathrm{C}-2)$ et C-3-bis) avec $\Delta_{n}=O\left(A_{n}^{-3 / 2}\right)$, alors :

iii) $\left\{\begin{array}{c}A_{n}^{-1 / 2} \operatorname{tr}\left(V_{n}^{-1}\left([M]_{n}-\langle M\rangle_{n}\right){ }^{*} V_{n}^{-1}\right) \underset{n \rightarrow \infty}{\longrightarrow} 0 \text { p.s. } \\ \text { et } \\ A_{n+1}^{-1 / 2} \sum_{k=0}^{n} a_{k} \operatorname{tr}\left\{V_{k}^{-1}\left([M]_{k}-\langle M\rangle_{k}\right)^{*} V_{k}^{-1}\right\} \underset{n \rightarrow \infty}{\longrightarrow} 0 \text { p.s. }\end{array}\right.$

iv) Si, de plus, l'hypothèse $H$-1) est vérifiée et la série

$$
\sum_{k \geq 0} A_{k+1}^{-1 / 2}\left[\mathbb{E}\left\{\left\|R^{1 / 2} V_{k+1}^{-1} \Delta M_{k+1}\right\|^{2} / \mathfrak{F}_{k}\right\}-a_{k} \operatorname{tr}\{C\}\right]
$$

est p.s. convergente, $R$ étant la solution de l'équation de Lyapounov $I=R U+{ }^{*} U R$, alors :

$$
\begin{aligned}
& A_{n}^{-1 / 2} \operatorname{tr}\left\{V_{n}^{-1}[M]_{n}{ }^{*} V_{n}^{-1}\right\} \underset{n \rightarrow \infty}{\longrightarrow} 0 \\
& A_{n}^{-1 / 2} \sum_{k=0}^{n} a_{k} \operatorname{tr}\left\{V_{k}^{-1}[M]_{k}{ }^{*} V_{k}^{-1}-C\right\} \underset{n \rightarrow \infty}{\longrightarrow} 0 \text { p.s. }
\end{aligned}
$$

La seconde propriété de iv) a également lieu si $V_{n}^{-1}\langle M\rangle_{n}{ }^{*} V_{n}^{-1}-C=O\left[A_{n}^{-\rho}\right]$ p.s. ou $\mathbb{E}\left(\left\|V_{n}^{-1}\langle M\rangle_{n}{ }^{*} V_{n}^{-1}-C\right\|\right)=O\left[A_{n}^{-\rho}\right]$ p.s. pour un $\rho>\frac{1}{2}$.

Preuve du lemme 5.8.

i) On a :

$$
\begin{aligned}
v_{n+1}^{-2}\left\{[M]_{n+1}-\langle M\rangle_{n+1}\right\}+\sum_{k=0}^{n}\left\{1-\left(\frac{v_{k}}{v_{k+1}}\right)^{2}\right\} & \frac{[M]_{k}-\langle M\rangle_{k}}{v_{k}^{2}} \\
& =\sum_{k=0}^{n} v_{k+1}^{-2}\left(\Delta M_{k+1}{ }^{*} \Delta M_{k+1}-\mathbb{E}\left\{\Delta M_{k+1}{ }^{*} \Delta M_{k+1} / \mathfrak{F}_{k}\right\}\right)
\end{aligned}
$$

$$
v_{n+1}^{-2}[M]_{n+1}+\sum_{k=0}^{n}\left\{1-\left(\frac{v_{k}}{v_{k+1}}\right)^{2}\right\}\left(\frac{[M]_{k}}{v_{k}^{2}}-C\right)=\sum_{k=0}^{n} v_{k+1}^{-2}\left\{\Delta M_{k+1} *\left(\Delta M_{k+1}\right)-\left(v_{k+1}^{2}-v_{k}^{2}\right) C\right\} .
$$

Par ailleurs, posant pour $u \in \mathbb{R}^{d}$ :

$N_{n+1}(u)=\sum_{k=0}^{n}\left(\log v_{k+1}^{2}\right)^{-1 / 2} v_{k+1}^{-2}\left(\left\langle u, \Delta M_{k+1}\right\rangle^{2}-\mathbb{E}\left\{\left\langle u, \Delta M_{k+1}\right\rangle^{2} / \mathfrak{F}_{k}\right\}\right)$,

on a :

$$
\mathbb{E}\left\{\left|\Delta N_{k+1}(u)\right|^{\beta} / \mathfrak{F}_{k}\right\}=O\left\{\left(\log v_{k+1}^{2}\right)^{-\frac{\beta}{2}} \mathbb{E}\left\{\left|v_{k+1}^{-1}\left\langle u, \Delta M_{k+1}\right\rangle\right|^{2 \beta} / \mathfrak{F}_{k}\right\}\right\} .
$$


Donc sous l'hypothèse H"-3), la série $\sum_{k \geq 0} \mathbb{E}\left\{\left|\Delta N_{k+1}(u)\right|^{\beta} / \mathfrak{F}_{k}\right\}$ converge p.s. et le théorème de Chow implique que la martingale $\left(N_{n}(u)\right)$ converge p.s. vers une limite finie. D'où les deux propriétés :

$$
\left(\log v_{n+1}^{2}\right)^{-1 / 2} \sum_{k=0}^{n} v_{k+1}^{-2}\left(\left\langle u, \Delta M_{k+1}\right\rangle^{2}-\mathbb{E}\left\{\left\langle u, \Delta M_{k+1}\right\rangle^{2} / \mathfrak{F}_{k}\right\}\right) \underset{n \rightarrow \infty}{\longrightarrow} 0 \text { p.s. }
$$

pour tout $u \in \mathbb{R}^{d}$; et

$$
\left(\log v_{n+1}^{2}\right)^{-1 / 2} v_{n+1}^{-2}\left([M]_{n+1}-\langle M\rangle_{n+1}\right) \underset{n \rightarrow \infty}{\longrightarrow} 0 \text { p.s. }
$$

par le lemme de Kronecker. La première propriété de la partie 1) du lemme découle de $(5.40,5.42)$ et $(5.43)$. ii) $\mathrm{Si}$, en outre, $\sum_{k \geq 0}\left(\log v_{k+1}^{2}\right)^{-1 / 2} v_{k+1}^{-2}\left(\mathbb{E}\left\{\Delta M_{k+1}{ }^{*} \Delta M_{k+1} / \mathfrak{F}_{k}\right\}-\left(v_{k+1}^{2}-v_{k}^{2}\right) C\right)=\xi$ est p.s. convergente, alors compte tenu de (5.41), on peut affirmer que :

$$
\begin{aligned}
\sum_{k=0}^{n} & v_{k+1}^{-2}\left(\left\langle u, \Delta M_{k+1}\right\rangle^{2}-\left(v_{k+1}^{2}-v_{k}^{2}\right){ }^{*} u C u\right) \\
& =\sum_{k=0}^{n} v_{k+1}^{-2}\left(\mathbb{E}\left\{\left\langle u, \Delta M_{k+1}\right\rangle^{2} / \mathfrak{F}_{k}\right\}-\left(v_{k+1}^{2}-v_{k}^{2}\right){ }^{*} u C u\right)+o\left\{\left(\log v_{n+1}^{2}\right)^{1 / 2}\right\} \\
& =o\left\{\left(\log v_{n+1}^{2}\right)^{1 / 2}\right\} \quad \text { p.s., } \forall u \in \mathbb{R}^{d} .
\end{aligned}
$$

D'où l'on déduit grâce à $(5.41,5.43)$ et l'hypothèse H-1) :

$$
\begin{aligned}
& \left(\log v_{n}^{2}\right)^{-1 / 2} v_{n}^{-2}[M]_{n} \underset{n \rightarrow \infty}{\longrightarrow} 0 \text { et } \\
& \left(\log v_{n+1}^{2}\right)^{-1 / 2} \sum_{k=0}^{n}\left\{1-\left(\frac{v_{k}}{v_{k+1}}\right)^{2}\right\}\left(v_{k}^{-2}[M]_{k}-C\right) \underset{n \rightarrow \infty}{\longrightarrow} 0 \text { p.s. }
\end{aligned}
$$

La seconde propriété de ii) est immédiate si au lieu de la convergence p.s. de la série $\xi$, on a :

$$
v_{n}^{-2}\langle M\rangle_{n}-C=O\left[\left(\log v_{n+1}^{2}\right)^{-^{\rho}}\right] \text { p.s. }
$$

ou

$$
\mathbb{E}\left(\left\|v_{n}^{-2}\langle M\rangle_{n}-C\right\|\right)=O\left[\left(\log v_{n+1}^{2}\right)^{-\rho}\right]
$$

pour un $\rho>\frac{1}{2}$.

En effet, dans ce cas la série $\sum_{n \geq 0}\left[1-\left(\frac{v_{n}}{v_{n+1}}\right)^{2}\right]\left(\log v_{n+1}^{2}\right)^{-1 / 2}\left(v_{n}^{-2}\langle M\rangle_{n}-C\right)$ est p.s. convergente; d'où la propriété cherchée par le lemme de Kronecker. La partie 1) du lemme est établie. 
iii) Dans le cadre de la partie 2) du lemme, on a les relations suivantes analogues à (5.40) et (5.41) respectivement, valables pour toute matrice $R$ symétrique et positive :

$$
\begin{array}{r}
\operatorname{tr}\left\{R^{1 / 2} V_{n+1}^{-1}\left([M]_{n+1}-\langle M\rangle_{n+1}\right){ }^{*} V_{n+1}^{-1} R^{1 / 2}\right\} \\
+\sum_{k=0}^{n} \operatorname{tr}\left\{\left(R-{ }^{*} \Lambda_{k} R \Lambda_{k}\right) V_{k}^{-1}\left([M]_{k}-\langle M\rangle_{k}\right){ }^{*} V_{k}^{-1}\right\} \\
=\sum_{k=0}^{n} \operatorname{tr}\left\{R^{1 / 2} V_{k+1}^{-1}\left(\Delta[M]_{k+1}-\Delta\langle M\rangle_{k+1}\right)^{*} V_{k+1}^{-1} R^{1 / 2}\right\}
\end{array}
$$

et

$$
\begin{aligned}
\operatorname{tr}\left\{R^{1 / 2} V_{n+1}^{-1}\left([M]_{n+1}\right){ }^{*} V_{n+1}^{-1} R^{1 / 2}\right\}+\sum_{k=0}^{n} \operatorname{tr}\left\{\left(R-{ }^{*} \Lambda_{k} R \Lambda_{k}\right)\left(V_{k}^{-1}[M]_{k}{ }^{*} V_{k}^{-1}-C\right)\right\} \\
=\sum_{k=0}^{n} \operatorname{tr}\left\{R^{1 / 2} V_{k+1}^{-1}\left[\Delta M_{k+1}{ }^{*}\left(\Delta M_{k+1}\right)-\left(V_{k+1} C^{*} V_{k+1}-V_{k} C^{*} V_{k}\right)\right]{ }^{*} V_{k+1}^{-1} R^{1 / 2}\right\}
\end{aligned}
$$

Si la condition C-3-bis) a lieu avec $\Delta_{n}=O\left(A_{n}^{-3 / 2}\right)$ et si $R$ est la solution de l'équation $R U+{ }^{*} U R=I$, on a :

$$
R-{ }^{*} \Lambda_{k} R \Lambda_{k}=a_{k} I+O\left(a_{k} A_{k}^{-3 / 2}\right) \quad(k \rightarrow \infty)
$$

Une adaptation des raisonnements effectués dans les étapes i) et ii) permet alors d'établir les propriétés de la partie 2) du lemme.

\section{e) Preuve du théorème 2.3 et du TLC du théorème $\mathbf{2 . 4}$}

D'après la partie 1) du lemme 5.6, on peut affirmer que

$$
\left\{A_{n}^{-1 / 2} H_{n}, V_{n}^{-1} M_{n}\right\} \underset{n \rightarrow \infty}{\stackrel{\mathcal{L}}{\longrightarrow}} \nu_{\infty}^{\prime} \otimes \mu_{\infty}
$$

où conditionnellement à $\{C=\Gamma\}, \nu_{\infty}^{\prime}$ est la loi d'une v.a. $\mathfrak{N}_{d \times d}(0, \widetilde{\Gamma} \otimes \Gamma)$ indépendante $\operatorname{de} C$ et $\widetilde{\Gamma}=U \Gamma+\Gamma^{*} U$. Compte tenu de (5.23), cette propriété implique que les v.a. $\left(D_{n}\right)$ définies par :

vérifient :

$$
D_{n}=V_{n}^{-1}\left(M_{n}{ }^{*} M_{n}-[M]_{n}\right) * V_{n}^{-1}
$$

$$
\left\{A_{n}^{-1 / 2} R^{1 / 2} D_{n+1} R^{1 / 2}+A_{n}^{-1 / 2} \sum_{k=0}^{n} R^{1 / 2}\left\{D_{k}-\Lambda_{k} D_{k}{ }^{*} \Lambda_{k}\right\} R^{1 / 2}, V_{n}^{-1} M_{n}\right\} \underset{n \rightarrow \infty}{\stackrel{\mathcal{L}}{\longrightarrow}} \widetilde{\nu}_{\infty} \otimes \mu_{\infty}
$$

où, pour toute matrice symétrique et positive $R, \widetilde{\nu}_{\infty}$ est la loi d'une v.a. de la forme $\Sigma^{\prime}(C)+{ }^{*} \Sigma^{\prime}(C), \Sigma^{\prime}(\Gamma)$ étant une v.a. gaussienne centrée, indépendante de $\left\{\eta, C,\left(\sum(x) ; x \in \mathfrak{X}\right)\right\}$ et de covariance $\widetilde{\Gamma} \otimes \Gamma$.

Or, on a : $A_{n}^{-1 / 2} D_{n+1} \underset{n \rightarrow \infty}{\longrightarrow} 0$ en probabilité, car d'une part la suite $\left(V_{n}^{-1} M_{n}\right)$ converge en loi et d'autre part la suite $\left(V_{n}^{-1}\langle M\rangle_{n}{ }^{*} V_{n}^{-1}\right)$ converge p.s. ce qui implique que $A_{n}^{-1 / 2} V_{n}^{-1}[M]_{n}{ }^{*} V_{n}^{-1} \underset{n \rightarrow \infty}{\longrightarrow} 0$ en probabilité. D'où la propriété suivante :

$$
\left\{A_{n}^{-1 / 2} \sum_{k=0}^{n} \operatorname{tr}\left\{\left(R-{ }^{*} \Lambda_{k} R \Lambda_{k}\right) D_{k}\right\}, V_{n}^{-1} M_{n}\right\} \underset{n \rightarrow \infty}{\stackrel{\mathcal{L}}{\longrightarrow}} \nu_{\infty} \otimes \mu_{\infty}
$$


où $\nu_{\infty}$ est la loi d'une v.a. de la forme $2 \sqrt{\operatorname{tr}\{\widetilde{C} R C R\}} G, G$ étant une v.a. gaussienne centrée, réduite et indépendante de $\left\{\eta, C,\left(\sum(x) ; x \in \mathfrak{X}\right)\right\}$. La partie 1 du théorème est établie.

Choisissons pour matrice $R$ la solution de l'équation de Lyapounov : $I=R U+{ }^{*} U R$. Alors grâce à la propriété :

$$
R-{ }^{*} \Lambda_{n} R \Lambda_{n}=a_{n} I+O\left(a_{n} A_{n}^{-3 / 2}\right) \text { et à la suivante }: D_{n}=o\left(A_{n}\right) \quad \text { p.s., }
$$

qui découle de $\mathrm{H}-3)$ ou H'-3), on peut affirmer que :

$$
\sum_{k=0}^{n} \operatorname{tr}\left\{\left(R-{ }^{*} \Lambda_{k} R \Lambda_{k}-a_{k} I\right) D_{k}\right\}=o\left(\sum_{k=0}^{n} a_{k} A_{k}^{-1 / 2}\right)=o\left(A_{n}^{1 / 2}\right) \text { p.s. }
$$

par conséquent (5.47) s'écrit :

$$
\left\{\frac{1}{\sqrt{A_{n}}} \sum_{k=1}^{n} a_{k} \operatorname{tr}\left(V_{k}^{-1}\left\{M_{k}{ }^{*} M_{k}-[M]_{k}\right\}{ }^{*} V_{k}^{-1}\right), V_{n}^{-1} M_{n}\right\} \underset{n \rightarrow \infty}{\stackrel{\mathcal{L}}{\longrightarrow}} \nu_{\infty} \otimes \mu_{\infty} .
$$

On en déduit la partie 2) du théorème 2.3. En effet, en vertu de l'une ou l'autre des deux hypothèses suivantes :

$\Delta A_{n}^{\rho}\left|\operatorname{tr}\left(V_{n}^{-1}\langle M\rangle_{n}{ }^{*} V_{n}^{-1}\right)-\operatorname{tr}(C)\right|=O(1)$ p.s.,

$\Delta A_{n}^{\rho} \mathbb{E}\left\{\left|\operatorname{tr}\left(V_{n}^{-1}\langle M\rangle_{n}{ }^{*} V_{n}^{-1}\right)-\operatorname{tr}(C)\right|\right\}=O(1)$,

pour un $\rho>\frac{1}{2}$, on peut substituer dans le TLC précédent la v.a. $\operatorname{tr}(C)$ à la suite $\left(\operatorname{tr}\left(V_{k}^{-1}[M]_{k}{ }^{*} V_{k}^{-1}\right)\right)$.

Plaçons nous sous les hypothèses du théorème 2.4, en gardant le même choix de la matrice $R$; et posons $\widetilde{D}_{k}=V_{k}^{-1}\left\{M_{k}{ }^{*} M_{k}-\langle M\rangle_{k}\right\}{ }^{*} V_{k}^{-1}$.

D'après la preuve du lemme 5.8 , l'hypothèse $H^{\prime}$ "-3) permet de substituer les v.a. $\left(\widetilde{D}_{k}\right)$ aux v.a. $\left(D_{k}\right)$ dans (5.47). Or , si l'événement $\left\{\varlimsup A_{n}^{-1 / 4}\left\|V_{n}^{-1} M_{n}\right\|=\infty\right\}$ est négligeable, $\widetilde{D}_{n}=O\left(A_{n}^{1 / 2}\right)$ p.s. et par suite :

$$
\sum_{k=0}^{n} \operatorname{tr}\left\{\left(R-{ }^{*} \Lambda_{k} R \Lambda_{k}-a_{k} I\right) \widetilde{D}_{k}\right\}=o\left(\sum_{k=0}^{n} a_{k} A_{k}^{-1 / 2}\right)=o\left(A_{n}^{1 / 2}\right) \text { p.s. }
$$

Ainsi l'écriture suivante de la propriété (5.47) est justifiée :

$$
\left\{A_{n}^{-1 / 2} \sum_{k=0}^{n} a_{k} \operatorname{tr}\left\{V_{k}^{-1}\left(M_{k}{ }^{*} M_{k}-\langle M\rangle_{k}\right){ }^{*} V_{k}^{-1}\right\}, V_{n}^{-1} M_{n}\right\} \underset{n \rightarrow \infty}{\stackrel{\mathcal{L}}{\longrightarrow}} \nu_{\infty} \otimes \mu_{\infty} .
$$

Elle implique le TLC du théorème 2.4, car d'après le lemme 5.8, chacune des hypothèses suivantes :

ムla série $\sum_{n \geq 0} A_{n+1}^{-1 / 2}\left[\mathbb{E}\left\{\left\|R^{1 / 2} V_{n+1}^{-1} \Delta M_{n+1}\right\|^{2} / \mathfrak{F}_{n}\right\}-a_{n} \operatorname{tr}\{C\}\right]$ converge p.s. ;

$\mathrm{ou}$

$$
\mathbf{\Delta} A_{n}^{\rho}\left|\operatorname{tr}\left(V_{n}^{-1}[M]_{n} * V_{n}^{-1}\right)-\operatorname{tr}(C)\right|=O(1)
$$

ou

$\mathbf{\Delta} A_{n}^{\rho} \mathbb{E}\left(\left|\operatorname{tr}\left(V_{n}^{-1}[M]_{n}{ }^{*} V_{n}^{-1}\right)-\operatorname{tr}(C)\right|\right)=O(1)$, pour un $\rho>\frac{1}{2} ;$

permet de substituer la matrice $C$ à $\left(V_{k}^{-1}\langle M\rangle_{k}{ }^{*} V_{k}^{-1}\right)$ dans la propriété ci-dessus.

\section{f) Preuve de la partie 1 du théorème 2.5}

La partie 2) du lemme 5.6 implique que le TLCG s'applique au couple de martingales normalisées $\left(\left(\log v_{n}^{2}\right)^{-1 / 2} K_{n}, \frac{M_{n}}{v_{n}}\right)$. Plus précisément :

$$
\left(\left(\log v_{n}^{2}\right)^{-1 / 2} K_{n}, \frac{M_{n}}{v_{n}}\right) \underset{n \rightarrow \infty}{\stackrel{\mathcal{L}}{\longrightarrow}} \nu_{\infty}^{\prime} \otimes \mu_{\infty}
$$


où conditionnellement à $\{C=\Gamma\}, \nu_{\infty}^{\prime}$ est la loi d'une v.a. $\mathfrak{N}_{d \times d}(0, \Gamma \otimes \Gamma)$ que l'on peut choisir indépendante de $C$; d'où l'on déduit la première propriété du théorème 2.5 grâce à $(5.24)$ et à la propriété $\left(\log v_{n}^{2}\right)^{-1 / 2} D_{n} \underset{n \rightarrow \infty}{\longrightarrow} 0$ en probabilité.

\section{g) Preuve du théorème 3.2}

D'après le lemme 5.6 et l'égalité (5.23), on peut affirmer que :

$$
\left\{\frac{1}{\sqrt{n}} D_{n+1}+\frac{1}{\sqrt{n}} \sum_{k=0}^{n}\left(D_{k}-\Lambda_{k} D_{k}{ }^{*} \Lambda_{k}\right), V_{n}^{-1} M_{n}\right\} \underset{n \rightarrow \infty}{\stackrel{\mathcal{L}}{\longrightarrow}}\left\{\Sigma^{\prime}(C)+{ }^{*} \Sigma^{\prime}(C), \Sigma(\eta)\right\}
$$

de manière stable, où $\sum^{\prime}(Y)$ est une v.a. gaussienne matricielle qu'on peut choisir indépendante du triplet $\left\{\eta, C,\left(\sum(x) ; x \in \mathfrak{X}\right)\right\}$ et dont la matrice de covariance est $\left(Y-\Lambda_{\infty} Y^{*} \Lambda_{\infty}\right) \otimes \Lambda_{\infty} Y^{*} \Lambda_{\infty}$ pour une matrice symétrique et positive $Y$. Comme $\frac{1}{\sqrt{n}} D_{n} \underset{n \rightarrow \infty}{\longrightarrow} 0$ en probabilité, on en déduit que pour toute matrice $R^{\prime}$ symétrique, positive et éventuellement aléatoire en tant que fonction mesurable du couple $(C, \eta)$ :

$$
\left\{\frac{1}{\sqrt{n}} \sum_{k=0}^{n} \operatorname{tr}\left\{\left(R^{\prime}-{ }^{*} \Lambda_{k} R^{\prime} \Lambda_{k}\right) D_{k}\right\}, V_{n}^{-1} M_{n}\right\} \underset{n \rightarrow \infty}{\stackrel{\mathcal{L}}{\longrightarrow}}\left\{\sigma(C) G, \sum(\eta)\right\}
$$

où $G$ est une v.a. gaussienne standard, indépendante du triplet

$\left\{C, \eta,\left(\sum(x) ; x \in \mathfrak{X}\right)\right\}$ et $\sigma^{2}(C)=4 \operatorname{tr}\left\{R^{\prime}\left[C-{ }^{*} \Lambda_{\infty} C \Lambda_{\infty}\right] R^{\prime} \Lambda_{\infty} C{ }^{*} \Lambda_{\infty}\right\}$.

Prenant : $R^{\prime}=\sum_{k=0}^{\infty}{ }^{*} \Lambda_{\infty}^{k} \Lambda_{\infty}^{k}$, on vérifie aisément que $R^{\prime}$ satisfait la relation $R^{\prime}-{ }^{*} \Lambda_{\infty} R^{\prime} \Lambda_{\infty}=I$. Or, celle-ci implique la suivante : $R^{\prime}-{ }^{*} \Lambda_{k} R^{\prime} \Lambda_{k}=I+o\left(k^{-3 / 2}\right)$ dès que la suite $\left(k^{3 / 2}\left(\Lambda_{k}-\Lambda_{\infty}\right)\right)$ est bornée. Ainsi, la partie 3) du théorème découle de celle qui la précède, car la propriété : $D_{k}=o(k)$ p.s. implique que :

$$
\frac{1}{\sqrt{n}} \sum_{k=0}^{n} k^{-3 / 2} D_{k} \underset{n \rightarrow \infty}{\longrightarrow} 0 \text { p.s. }
$$

\section{h) Preuves des TLC des théorèmes 3.3 et 3.4}

1) Montrons la partie 1) du théorème 3.3. La relation (5.23) et la suivante

$$
\begin{array}{r}
V_{n+1}^{-1}\left([M]_{n+1}-\langle M\rangle_{n+1}\right){ }^{*} V_{n+1}^{-1}+\sum_{k=0}^{n}\left(V_{k}^{-1}\left\{[M]_{k}-\langle M\rangle_{k}\right\}{ }^{*} V_{k}^{-1}-V_{k+1}^{-1}\left\{[M]_{k}-\langle M\rangle_{k}\right\}{ }^{*} V_{k+1}^{-1}\right) \\
+\sum_{k=0}^{n} V_{k+1}^{-1}\left\{\Delta M_{k+1}{ }^{*} \Delta M_{k+1}-\Delta\langle M\rangle_{k+1}\right\}{ }^{*} V_{k}^{-1}=\bar{H}_{n+1},
\end{array}
$$

donnent :

$$
\begin{gathered}
V_{n+1}^{-1}\left(M_{n+1}{ }^{*} M_{n+1}-\langle M\rangle_{n+1}\right){ }^{*} V_{n+1}^{-1} \\
+\sum_{k=0}^{n}\left(V_{k}^{-1}\left\{M_{k}{ }^{*} M_{k}-\langle M\rangle_{k}\right\}{ }^{*} V_{k}^{-1}-V_{k+1}^{-1}\left\{M_{k}{ }^{*} M_{k}-\langle M\rangle_{k}\right\}{ }^{*} V_{k+1}^{-1}\right) \\
=H_{n+1}+{ }^{*} H_{n+1}+\bar{H}_{n+1} .
\end{gathered}
$$

Ainsi, les v.a. $\widetilde{D}_{k}=V_{k}^{-1}\left\{M_{k}{ }^{*} M_{k}-\langle M\rangle_{k}\right\}{ }^{*} V_{k}^{-1}$ vérifient la relation :

$$
\widetilde{D}_{n+1}+\sum_{k=0}^{n}\left(\widetilde{D}_{k}-\Lambda_{k} \widetilde{D}_{k}^{*} \Lambda_{k}\right)=H_{n+1}+{ }^{*} H_{n+1}+\bar{H}_{n+1} .
$$


Vu que :

$$
\left\|\widetilde{D}_{n+1}\right\|=O\left(\left\|V_{n+1}^{-1} M_{n+1}\right\|^{2}\right)=o\left(n^{1 / 2}\right) \text { p.s. }
$$

en vertu de l'hypothèse H-5), la partie 1) du théorème 3.3 est une conséquence directe du lemme 5.7.

2) Pour obtenir la partie 2) du théorème 3.3, on remarque d'abord que pour toute matrice $R^{\prime}$ symétrique, positive et éventuellement aléatoire en tant que fonction mesurable du couple $(\eta, C)$ :

$$
\begin{aligned}
n^{-1 / 2} \sum_{k=0}^{n} \operatorname{tr}\left\{\left(R^{\prime}-{ }^{*} \Lambda_{k} R^{\prime} \Lambda_{k}\right) \widetilde{D}_{k}\right\} \\
\quad=n^{-1 / 2} \sum_{k=0}^{n} \operatorname{tr}\left\{\left(R^{\prime}\right)^{1 / 2}\left(\widetilde{D}_{k}-\Lambda_{k} \widetilde{D}_{k}{ }^{*} \Lambda_{k}\right)\left(R^{\prime}\right)^{1 / 2}\right\} \\
\quad=n^{-1 / 2} \operatorname{tr}\left\{\left(R^{\prime}\right)^{1 / 2}\left(H_{n+1}+{ }^{*} H_{n+1}+\bar{H}_{n+1}\right)\left(R^{\prime}\right)^{1 / 2}\right\}+o(1) . \\
=n^{-1 / 2} \operatorname{tr}\left\{R^{\prime}\left(H_{n+1}+{ }^{*} H_{n+1}+\bar{H}_{n+1}\right)\right\}+o(1) \\
=n^{-1 / 2}\left\langle\operatorname{Vect}\left(R^{\prime}\right), \operatorname{Vect}\left(H_{n+1}+{ }^{*} H_{n+1}+\bar{H}_{n+1}\right)\right\rangle+o(1) \\
=2\left\langle\operatorname{Vect}\left(R^{\prime}\right), \mathcal{H}_{n+1}\right\rangle+\left\langle\operatorname{Vect}\left(R^{\prime}\right), \overline{\mathcal{H}}_{n+1}\right\rangle+o(1) \\
=\left\langle\left(\begin{array}{c}
2 \operatorname{Vect}\left(R^{\prime}\right) \\
\operatorname{Vect}\left(R^{\prime}\right)
\end{array}\right), n^{-1 / 2} \mathbb{H}_{n+1}\right\rangle+o(1) \text { p.s. },(n \rightarrow \infty),
\end{aligned}
$$

$\operatorname{car} \operatorname{tr}(A B)=\left\langle\operatorname{Vect}\left({ }^{*} A\right)\right.$, Vect $\left.(B)\right\rangle$.

La convergence en loi de la suite $\left\{n^{-1 / 2} \mathbb{H}_{n+1}, V_{n}^{-1} M_{n}\right\}$ vers $\left\{\left(\begin{array}{c}\sum^{\prime}(C) \\ \sum^{\prime \prime}(C)\end{array}\right), \sum(\eta)\right\}$ établie au lemme 5.7 est évidemment stable, on peut donc affirmer que :

$$
\left\{\frac{1}{\sqrt{n}} \sum_{k=0}^{n} \operatorname{tr}\left\{\left(R^{\prime}-{ }^{*} \Lambda_{k} R^{\prime} \Lambda_{k}\right) \widetilde{D}_{k}\right\}, V_{n}^{-1} M_{n}\right\} \underset{n \rightarrow \infty}{\stackrel{\mathcal{L}}{\longrightarrow}}\left\{\widetilde{\sigma}(C) G, \sum(\eta)\right\}
$$

où $G$ est une v.a. gaussienne standard, indépendante du triplet $\left\{\eta, C,\left(\sum(x) ; x \in \mathfrak{X}\right)\right\}$ et

$$
\tilde{\sigma}^{2}\left(C, R^{\prime}\right)=\tilde{\sigma}^{2}(C)=*\left(\begin{array}{c}
2 \operatorname{Vect}\left(R^{\prime}\right) \\
\operatorname{Vect}\left(R^{\prime}\right)
\end{array}\right) \triangle(C)\left(\begin{array}{c}
2 \operatorname{Vect}\left(R^{\prime}\right) \\
\operatorname{Vect}\left(R^{\prime}\right)
\end{array}\right) \text {; }
$$

$\triangle(Y)$ étant la matrice définie par (3.2).

Désormais, supposons que la suite $\left(n\left(\Lambda_{n}-\Lambda_{\infty}\right)\right)$ est bornée. Alors on peut remplacer dans le résultat précédent la suite $\left(\frac{1}{\sqrt{n}} \sum_{k=0}^{n} \operatorname{tr}\left\{\left(R^{\prime}-{ }^{*} \Lambda_{k} R^{\prime} \Lambda_{k}\right) \widetilde{D}_{k}\right\}\right)$ par la suite $\left(\frac{1}{\sqrt{n}} \sum_{k=0}^{n} \operatorname{tr}\left\{\left(R^{\prime}-{ }^{*} \Lambda_{\infty} R^{\prime} \Lambda_{\infty}\right) \widetilde{D}_{k}\right\}\right)$, car leur différence tend vers 0 lorsque $n \rightarrow \infty$. Or, pour $R^{\prime}=\sum_{k=0}^{\infty}{ }^{*} \Lambda_{\infty}^{k} \Lambda_{\infty}^{k}$, on a : $R^{\prime}-{ }^{*} \Lambda_{\infty} R^{\prime} \Lambda_{\infty}=I$. D'où la partie $3 \mathrm{du}$ théorème 3.3 .

3) Si, en plus de la bornitude de la suite $\left(n\left(\Lambda_{n}-\Lambda_{\infty}\right)\right)$, la version suivante de l'hypothèse H-1) a lieu :

$$
V_{n}^{-1}\langle M\rangle_{n}{ }^{*} V_{n}^{-1}=C+O\left(n^{-\rho}\right) \text { p.s. pour un } \rho>1 ; \text { et } C \text { est p.s. inversible, }
$$

alors on peut substituer la v.a. $C$ à la suite $\left(V_{n}^{-1}\langle M\rangle_{n}{ }^{*} V_{n}^{-1}\right)$ dans les résultats des parties 2) et 3) du théorème 3.3. Autrement dit, on a la propriété suivante :

$$
\left\{\frac{1}{\sqrt{n}} \sum_{k=0}^{n} \operatorname{tr}\left\{\left(R^{\prime}-{ }^{*} \Lambda_{\infty} R^{\prime} \Lambda_{\infty}\right)\left(V_{k}^{-1} M_{k}{ }^{*} M_{k}{ }^{*} V_{k}^{-1}-C\right)\right\}, V_{n}^{-1} M_{n}\right\} \underset{n \rightarrow \infty}{\stackrel{\mathcal{L}}{\longrightarrow}}\left\{\tilde{\sigma}\left(C, R^{\prime}\right) G, \sum(\eta)\right\}
$$


Mais comme $C$ est inversible, on peut prendre $R^{\prime}=\sum_{k=0}^{\infty}{ }^{*} \Lambda_{\infty}^{k} C^{-1} \Lambda_{\infty}^{k}$ dans la propriété ci-dessus. La matrice $R^{\prime}$ vérifie alors : $R^{\prime}-{ }^{*} \Lambda_{\infty} R^{\prime} \Lambda_{\infty}=C^{-1}$; d'où la partie 1) du théorème 3.4 car :

$$
\begin{aligned}
\frac{1}{\sqrt{n}}\left(\sum_{k=1}^{n}{ }^{*} M_{k} Q_{k}^{-1} M_{k}-\sum_{k=1}^{n}{ }^{*} M_{k}{ }^{*} V_{k}^{-1} C^{-1} V_{k}^{-1} M_{k}\right) \\
\quad=o\left(n^{-1 / 2} \sum_{k=1}^{n} k^{(1 / 2-\rho)}\right)=o\left(n^{(1-\rho)}\right)=o(1) \text { p.s. }(i . e \rho>1) .
\end{aligned}
$$

\subsubsection{Lois du logarithme itéré}

La dernière assertion du théorème 2.4 et la partie 2-b) du théorèmes 2.5 découlent de la loi du logarithme itéré établie dans $[4,7]$ et du lemme 5.8 .

\section{i) Preuve de la LLI du théorème $\mathbf{2 . 4}$}

D'après la relation (5.23), on a :

$$
\sum_{k=0}^{n} \operatorname{tr}\left\{\left(R-{ }^{*} \Lambda_{k} R \Lambda_{k}\right) D_{k}\right\}+\operatorname{tr}\left\{R D_{n+1}\right\}=2 \sum_{k=0}^{n}\left\langle R^{1 / 2} V_{k+1}^{-1} M_{k}, R^{1 / 2} V_{k+1}^{-1} \Delta M_{k+1}\right\rangle=2 \widetilde{L}_{n+1}
$$

où : $\quad D_{n}=V_{n}^{-1}\left(M_{n}{ }^{*} M_{n}-[M]_{n}\right){ }^{*} V_{n}^{-1}$.

Par ailleurs, si l'événement $\Delta=\left\{\varlimsup \lim A_{n}^{-1 / 4}\left\|V_{n}^{-1} M_{n}\right\|=\infty\right\}$ est négligeable, l'hypothèse H"-3) permet d'appliquer la loi du logarithme itéré rappelée ci-dessus à la martingale scalaire $\left(\widetilde{L}_{n}\right)$ :

$$
\varlimsup_{n \rightarrow \infty}\left(2\langle\widetilde{L}\rangle_{n} \log \log \langle\widetilde{L}\rangle_{n}\right)^{-1 / 2}\left|\widetilde{L}_{n}\right|=1 \text { p.s. }
$$

d'où :

$$
\varlimsup_{n \rightarrow \infty}\left(2 A_{n} \log \log A_{n}\right)^{-1 / 2}\left|\widetilde{L}_{n}\right|=\sqrt{\operatorname{tr}\{\widetilde{C} R C R\}} \text { p.s. },
$$

car d'après la partie $1 \mathrm{du}$ lemme 5.2 :

$$
A_{n}^{-1}\left\langle\widetilde{L}_{n}\right\rangle \underset{n \rightarrow \infty}{\longrightarrow} \operatorname{tr}\{\widetilde{C} R C R\} \text { p.s. }
$$

On conclut en exploitant la partie 2) du lemme 5.8, la relation (5.51), la propriété (5.48) vérifiée par la matrice $R$ et en remarquant que sous $\mathrm{H}-1$ ) et $\mathrm{H}^{\prime}-2$ ) on a $\mathrm{H}-2$ ) et $\left.\mathrm{H}-4\right)$.

\section{j) Preuve de la LLI du théorème 2.5}

Soient $x, y$ deux vecteurs de la base canonique de $\mathbb{R}^{d}$. Si l'événement $\Delta=\left\{\varlimsup\left(\log v_{n}^{2}\right)^{-1 / 4} v_{n}^{-2}\left\|M_{n}\right\|=\infty\right\}$ est négligeable. L'hypothèse $\mathrm{H}$ "-3) assure d'après le théorème 2.4 de [4]que la martingale scalaire $\left({ }^{*} x K_{n} y\right)$ vérifie la loi du logarithme itéré. On en déduit la loi LLI avec poids suivante, compte tenu de la partie 2) du lemme 5.3 et de la relation (5.24) :

$$
\begin{gathered}
\varlimsup_{n \rightarrow \infty}\left(2 \log v_{n}^{2} \log \log \log v_{n}^{2}\right)^{-1 / 2}\left|\sum_{k=1}^{n}\left(1-\frac{v_{k}^{2}}{v_{k+1}^{2}}\right) v_{k}^{-2}\left\{\left\langle x, M_{k}\right\rangle\left\langle y, M_{k}\right\rangle-{ }^{*} x[M]_{k} y\right\}\right| \\
=\sqrt{2\left({ }^{*} x C x\right)^{2}+2{ }^{*} x C x^{*} y C y} \text { p.s. }
\end{gathered}
$$


Mais d'après la partie 1) du lemme 5.8, la convergence p.s. de la série

$$
\sum_{n \geq 0}\left(\log v_{n+1}^{2}\right)^{-1 / 2} v_{n+1}^{-2}\left[\mathbb{E}\left\{\Delta M_{n+1} * \Delta M_{n+1} / \mathfrak{F}_{n}\right\}-\left(v_{n+1}^{2}-v_{n}^{2}\right) C\right]
$$

ou bien l'une ou l'autre des deux conditions : $v_{n}^{-2}\langle M\rangle_{n}-C=O\left[\left(\log v_{n+1}^{2}\right)^{-{ }^{\rho}}\right]$ p.s. ou $\mathbb{E}\left(\left\|v_{n}^{-2}\langle M\rangle_{n}-C\right\|\right)=O\left[\left(\log v_{n+1}^{2}\right)^{-\rho}\right]$ pour un $\rho>\frac{1}{2}$, permet de substituer ${ }^{*} x C y$ à $\frac{{ }^{*} x[M]_{k} y}{v_{k}^{2}}$ dans la relation précédente. La partie 2-b) du théorème 2.5 est établie. Elle reste vraie en substituant H'-2) au couple $\{\mathrm{H}-2), \mathrm{H}-4)\}$ car les hypothèses H-1) et H'-2) impliquent H-2) et H-4). On l'obtient enfin sous H-1) et l'hypothèse :

$$
\left.\left.\sum_{n \geq 0} \mathbb{E}\left\{\left\|v_{n+1}^{-1} \Delta M_{n+1}\right\|^{2 \beta} / \mathfrak{F}_{n}\right\}<\infty \text { p.s. pour un réel } \beta \in\right] 1,2\right]
$$

car dans ce cas on a H'-2), H"-3) et la loi du logarithme itéré :

$$
\varlimsup \frac{\left\|M_{n}\right\|}{\sqrt{2 v_{n}^{2} \log \log v_{n}^{2}}} \leq \operatorname{tr}(C) \text { p.s. ; }
$$

ce qui implique que $\Delta$ est négligeable.

\section{k) Preuve de la LLI du théorème 3.4}

Compte tenu de la partie $h$ ) de 5.2.2, posant $\widetilde{D}_{k}=V_{k}^{-1}\left\{M_{k}{ }^{*} M_{k}-\langle M\rangle_{k}\right\}{ }^{*} V_{k}^{-1}$, la propriété : $n^{-1 / 2} \sum_{k=0}^{n} \operatorname{tr}\left\{\left(R^{\prime}-{ }^{*} \Lambda_{\infty} R^{\prime} \Lambda_{\infty}\right) \widetilde{D}_{k}\right\}=\left\langle\left(\begin{array}{c}2 \operatorname{Vect}\left(R^{\prime}\right) \\ \operatorname{Vect}\left(R^{\prime}\right)\end{array}\right), n^{-1 / 2} \mathbb{H}_{n+1}\right\rangle+o(1)$ p.s., $(n \rightarrow \infty)$,

a lieu pour toute matrice $R^{\prime}$ symétrique, positive, éventuellement aléatoire en tant que fonction mesurable du couple $(\eta, C)$, dès que la suite $\left(n\left(\Lambda_{n}-\Lambda_{\infty}\right)\right)$ est bornée et on a : $\left\|V_{n}^{-1} M_{n}\right\|=o\left(n^{1 / 4}\right)$ p.s. $(n \rightarrow \infty)$. Prenons $R^{\prime}=\sum_{k=0}^{\infty} * \Lambda_{\infty}^{k} C^{-1} \Lambda_{\infty}^{k}$ dans la relation ci-dessus et supposons que l'hypothèse H"'-3) a lieu, à savoir :

$$
\left.\left.\sum_{n \geq 0} k^{-\beta} \mathbb{E}\left\{\left\|X_{n+1}\right\|^{4} / \mathfrak{F}_{n}\right\}<\infty \text { p.s. pour un } \beta \in\right] 1,2\right] .
$$

Alors les v.a. $\left(\left\langle\left(\begin{array}{c}2 \operatorname{Vect}\left(R^{\prime}\right) \\ \operatorname{Vect}\left(R^{\prime}\right)\end{array}\right), \mathbb{H}_{n+1}\right\rangle\right)$ vérifient la propriété suivante :

$$
\left\langle\left(\begin{array}{c}
2 \operatorname{Vect}\left(R^{\prime}\right) \\
\operatorname{Vect}\left(R^{\prime}\right)
\end{array}\right), \mathbb{H}_{n+1}\right\rangle=\bar{L}_{n+1}+o(\sqrt{n}) \text { p.s. }
$$

où :

$$
\begin{aligned}
& \bar{L}_{n+1}=2 \sum_{k=0}^{n}\left\langle\left(R_{k}^{\prime}\right)^{1 / 2} V_{k+1}^{-1} M_{k},\left(R_{k}^{\prime}\right)^{1 / 2} X_{k+1}\right\rangle \\
& +\sum_{k=0}^{n}\left(\left\|\left(R_{k}^{\prime}\right)^{1 / 2} X_{k+1}\right\|^{2}-\mathbb{E}\left\{\left\|\left(R_{k}^{\prime}\right)^{1 / 2} X_{k+1}\right\|^{2} / \mathfrak{F}_{k}\right\}\right),
\end{aligned}
$$

avec :

$$
R_{k}^{\prime}=\sum_{l=0}^{k} * \Lambda_{\infty}^{l} \bar{C}_{k}^{-1} \Lambda_{\infty}^{l} \text { et } \bar{C}_{k}=V_{k}^{-1}\left(I+\langle M\rangle_{k}\right) * V_{k}^{-1}
$$


En effet, grâce à l'hypothèse : $V_{n}^{-1}\langle M\rangle_{n}{ }^{*} V_{n}^{-1}=C+O\left(n^{-\rho}\right)$ p.s. pour un $\rho>1$; et au fait que $C$ est p.s. inversible, on a :

d'où :

$$
R^{\prime}=R_{k}^{\prime}+\sum_{l=0}^{k}{ }^{*} \Lambda_{\infty}^{l}\left(C^{-1}-\bar{C}_{k}^{-1}\right) \Lambda_{\infty}^{l}+\sum_{l>k}{ }^{*} \Lambda_{\infty}^{l} C^{-1} \Lambda_{\infty}^{l}=R_{k}^{\prime}+O\left(k^{-\rho}\right) \text { p.s. ; }
$$

$$
\begin{aligned}
\left\langle\left(\begin{array}{c}
2 \operatorname{Vect}\left(R^{\prime}\right) \\
\operatorname{Vect}\left(R^{\prime}\right)
\end{array}\right)\right. & \left., \mathbb{H}_{n+1}\right\rangle-\bar{L}_{n+1}=\sum_{k=0}^{n}\left\langle\left(R^{\prime}-R_{k}^{\prime}\right) V_{k+1}^{-1} M_{k}, X_{k+1}\right\rangle \\
& +\sum_{k=0}^{n}\left(\left\langle\left(R^{\prime}-R_{k}^{\prime}\right) X_{k+1}, X_{k+1}\right\rangle-\mathbb{E}\left\{\left\langle\left(R^{\prime}-R_{k}^{\prime}\right) X_{k+1}, X_{k+1}\right\rangle / \mathfrak{F}_{k}\right\}\right) \\
& =o\left(\sum_{k=0}^{n} k^{-\rho} k^{1 / 2}\right)=o\left(n^{\frac{3}{2}-\rho}\right)=o(\sqrt{n}) \text { p.s. }
\end{aligned}
$$

car d'une part $\rho>1$ et d'autre part $\left\|V_{k+1}^{-1} M_{k}\right\|\left\|X_{k+1}\right\|=o(\sqrt{k})$ et $\left\|X_{k+1}\right\|^{2}=o(\sqrt{k})$ p.s.

Or, la martingale $\left(\bar{L}_{n}\right)$ vérifie la LLI :

$$
\varlimsup_{n \rightarrow \infty}\left(2\langle\bar{L}\rangle_{n} \log \log \langle\bar{L}\rangle_{n}\right)^{-1 / 2}\left|\bar{L}_{n}\right|=1 \text { p.s. }
$$

cela résulte du fait que les propriétés : $\left\|V_{n}^{-1} M_{n}\right\|=o\left(n^{1 / 4}\right)$ p.s. et $\left.\mathrm{H}^{\prime \prime}-3\right)$ impliquent que la série :

$$
\begin{aligned}
& \sum_{n \geq 0} \mathbb{E}\left\{\left|\frac{\Delta \bar{L}_{n+1}}{\sqrt{\langle\bar{L}\rangle_{n+1}}}\right|^{4} / \mathfrak{F}_{n}\right\}<\infty \text { p.s. Comme }: n^{-1}\langle\bar{L}\rangle_{n} \underset{n \rightarrow \infty}{\longrightarrow} \widetilde{\sigma}^{2}\left(C, R^{\prime}\right) \text { p.s., on a la LLI : } \\
& \varlimsup_{n \rightarrow \infty}(2 n \log \log n)^{-1 / 2}\left|\left\langle\left(\begin{array}{c}
2 \operatorname{Vect}\left(R^{\prime}\right) \\
\operatorname{Vect}\left(R^{\prime}\right)
\end{array}\right), \mathbb{H}_{n}\right\rangle\right| \\
& =\varlimsup_{n \rightarrow \infty}(2 n \log \log n)^{-1 / 2}\left|\sum_{k=1}^{n}\left({ }^{*} M_{k}{ }^{*} V_{k}^{-1} C^{-1} V_{k}^{-1} M_{k}-d\right)\right|=\widetilde{\sigma}\left(C, R^{\prime}\right) \text { p.s. }
\end{aligned}
$$

La partie 2) du théorème en découle en vertu de la propriété (5.50).

\subsection{Preuve succinte du théorème 4.2}

\section{a) Calcul préliminaire}

Posons :

$$
\begin{aligned}
& \widetilde{\mathfrak{D}}_{n}=V_{n}^{-1}\left(M_{n}{ }^{*} M_{n}-\langle M\rangle_{n}\right){ }^{*} V_{n}^{-1} ; \widetilde{\mathfrak{D}}_{n}(1)=v_{n}^{-2}\left(M_{n}(1)^{*} M_{n}(1)-\langle M(1)\rangle_{n}\right) \\
& \widetilde{\mathfrak{D}}_{n}(2)=V_{n}^{-1}(2)\left(M_{n}(2)^{*} M_{n}(2)-\langle M(2)\rangle_{n}\right)^{*} V_{n}^{-1}(2) ; \widetilde{V}_{n}(2)=n^{\alpha / 2} V_{n}(2), n \geq 1
\end{aligned}
$$

En écrivant les relations (5.24) et (5.40) pour le couple $\left\{\left(M_{n}(1)\right),\left(v_{n}\right)\right\}$ et les relations (5.23) et (5.49) pour le couple $\left\{\left(M_{n}(2)\right),\left(\widetilde{V}_{n}(2)\right)\right\}$, on obtient les relations (5.53) et (5.54) ci-dessous, à savoir :

$$
\widetilde{\mathfrak{D}}_{n}(1)+\sum_{k=1}^{n}\left(1-\frac{v_{k}^{2}}{v_{k+1}^{2}}\right) \widetilde{\mathfrak{D}}_{k}(1)=K_{n+1}(1)+{ }^{*} K_{n+1}(1)+\bar{K}_{n+1}(1)
$$

où

$$
\begin{aligned}
& K_{n+1}(1)=\sum_{k=0}^{n} v_{k+1}^{-2} M_{k}(1)^{*}\left(\Delta M_{k+1}(1)\right), \\
& \bar{K}_{n+1}(1)=\sum_{k=0}^{n} v_{k+1}^{-2}\left(\Delta[M(1)]_{k+1}-\Delta\langle M(1)\rangle_{k+1}\right) .
\end{aligned}
$$




$$
\begin{aligned}
&(n+1)^{-\alpha} \widetilde{\mathfrak{D}}_{n}(2)+\sum_{k=1}^{n}\left(k^{-\alpha} \widetilde{\mathfrak{D}}_{k}(2)-(k+1)^{-\alpha} \Lambda_{k}(2) \widetilde{\mathfrak{D}}_{k}(2) * \Lambda_{k}(2)\right) \\
&=\sum_{k=1}^{n}(k+1)^{-\alpha}\left(\Delta H_{k+1}(2)+{ }^{*} \Delta H_{k+1}(2)\right)+\sum_{k=1}^{n}(k+1)^{-\alpha} \Delta \bar{H}_{k+1}(2)
\end{aligned}
$$

où

$$
\begin{aligned}
& H_{n+1}(2)=\sum_{k=0}^{n} V_{k+1}^{-1}(2) M_{k}(2) *\left(V_{k+1}^{-1}(2) \Delta M_{k+1}(2)\right) \\
& \bar{H}_{n+1}(2)=\sum_{k=0}^{n} V_{k+1}^{-1}(2)\left(\Delta[M(2)]_{k+1}-\Delta\langle M(2)\rangle_{k+1}\right) * V_{k+1}^{-1}(2) .
\end{aligned}
$$

Par ailleurs, la v.a. matricielle $R^{\prime}(2)=\sum_{k=0}^{\infty} * \Lambda_{\infty}^{k}(2) C^{-1}(2) \Lambda_{\infty}^{k}(2)$ vérifie la relation : $R^{\prime}(2)-{ }^{*} \Lambda_{\infty}(2) R^{\prime}(2) \Lambda_{\infty}(2)$ $=C^{-1}(2)$; laquelle implique la suivante en vertu de la condition (4.4) :

$$
R^{\prime}(2)-{ }^{*} \Lambda_{k}(2) R^{\prime}(2) \Lambda_{k}(2)=C^{-1}(2)+O\left(k^{-\alpha / 2}\right) .
$$

Compte tenu de (5.54), des propriétés précédentes de la v.a. $R^{\prime}(2)$ et des hypothèses H-5) et (4.5) sur le couple $\left\{\left(M_{n}(2)\right),\left(V_{n}(2)\right)\right\}$, on a :

$$
\begin{aligned}
\sum_{k=1}^{n} k^{-\alpha *} M_{k}(2)^{*} V_{k}^{-1}(2) C^{-1}(2) V_{k}^{-1}(2) & M_{k}(2) \\
= & \operatorname{tr}\left\{R^{\prime}(2) \sum_{k=0}^{n}(k+1)^{-\alpha} \Delta\left(2 H_{k+1}(2)+\bar{H}_{k+1}(2)\right)\right\}+o\left(n^{\frac{1-\alpha}{2}}\right) \text { p.s. }
\end{aligned}
$$

De même, la relation (5.53), la condition (4.3) sur la normalisation $\left(v_{n}\right)$ et les hypothèses (4.5) et (4.6) sur le couple $\left\{\left(M_{n}(1)\right),\left(v_{n}\right)\right\}$ permettent de justifier la relation suivante :

$$
s^{2} \sum_{k=1}^{n} k^{-\alpha}\left(v_{k}^{-2 *} M_{k}(1) C^{-1}(1) M_{k}(1)-d_{1}\right)=2 \operatorname{tr}\left\{C^{-1}(1) K_{n+1}(1)\right\}+o\left(n^{\frac{1-\alpha}{2}}\right) \text { p.s. }
$$

Remarquons maintenant qu'en posant : $Q_{n}=I+\langle M\rangle_{n}$, on a :

$$
\begin{aligned}
& { }^{*} M_{n} Q_{n}^{-1} M_{n}-d=\operatorname{tr}\left\{Q_{n}^{-1}\left(M_{n}{ }^{*} M_{n}-Q_{n}\right)\right\}=\operatorname{tr}\left\{{ }^{*} V_{n} Q_{n}^{-1} V_{n} \widetilde{\mathfrak{D}}_{n}\right\}-\operatorname{tr}\left\{Q_{n}^{-1}\right\} \\
& =\left(v_{n}^{-2 *} M_{n}(1) C^{-1}(1) M_{n}(1)-d_{1}\right)+\left({ }^{*} M_{k}(2){ }^{*} V_{k}^{-1}(2) C^{-1}(2) V_{k}{ }^{-1}(2) M_{k}(2)-d_{2}\right) \\
& \quad-\operatorname{tr}\left\{Q_{n}^{-1}\right\}-\operatorname{tr}\left\{\left({ }^{*} V_{n} Q_{n}^{-1} V_{n}-C^{-1}\right) \widetilde{\mathfrak{D}}_{n}\right\} .
\end{aligned}
$$

D'où l'on déduit, compte tenu de (5.55) et (5.56) :

$$
\begin{aligned}
\sum_{k=1}^{n} k^{-\alpha}\left({ }^{*} M_{k} Q_{k}^{-1} M_{k}-d\right)=\frac{2}{s^{2}} \operatorname{tr}\left\{C^{-1}(1)\right. & \left.K_{n+1}(1)\right\} \\
& +\operatorname{tr}\left\{R^{\prime}(2) \sum_{k=0}^{n}(k+1)^{-\alpha} \Delta\left(2 H_{k+1}(2)+\bar{H}_{k+1}(2)\right)\right\}+\zeta_{n}
\end{aligned}
$$


avec :

$$
\zeta_{n}=o\left(n^{\frac{1-\alpha}{2}}\right)-\sum_{k=1}^{n} k^{-\alpha} \operatorname{tr}\left\{Q_{k}^{-1}\right\}+\sum_{k=1}^{n} k^{-\alpha} \operatorname{tr}\left\{\widetilde{\mathfrak{D}}_{k} C^{-1}\left(V_{k}^{-1} Q_{k}{ }^{*} V_{k}^{-1}-C\right)\left({ }^{*} V_{k} Q_{k}^{-1} V_{k}\right)\right\} .
$$

\section{b) Preuve de l'assertion 1) du théorème}

L'hypothèse (4.5) sur le couple $(\mathrm{M}, \mathrm{V})$ implique l'assertion 1$)$ du théorème, à savoir :

$$
V_{n}^{-1} Q_{n}{ }^{*} V_{n}^{-1}=C+o\left(n^{-\frac{1-\alpha}{2}}\right) \text { p.s. avec } C=\operatorname{Diag}\{C(1), C(2)\}
$$

En effet :

\ $v_{n}^{-2} Q_{n}(1,1)=v_{n}^{-2}\left(I_{d_{1}}+\langle M(1)\rangle_{n}\right)=C(1)+o\left[\left(\log v_{n}^{2}\right)^{-\rho_{1}}\right]=C(1)+o\left(n^{-\frac{1-\alpha}{2}}\right)$ p.s. car : $\log v_{n}^{2} \sim s^{2} n^{1-\alpha}$ et $\rho_{1}>\frac{1}{2}$;

\ $V_{n}^{-1}(2) Q_{n}(2,2){ }^{*} V_{n}^{-1}(2)=V_{n}^{-1}(2)\left(I_{d_{2}}+\langle M(2)\rangle_{n}\right){ }^{*} V_{n}^{-1}(2)=C(1)+O\left(n^{-\rho_{2}}\right)$ p.s. et $\rho_{2}>1$;

\ $v_{n}^{-1} Q_{n}(1,2){ }^{*} V_{n}^{-1}(2)=v_{n}^{-1}\langle M\rangle_{n}(1,2){ }^{*} V_{n}^{-1}(2)$

$\operatorname{car} \alpha>\frac{1}{2}$.

$$
\begin{aligned}
& =\sum_{k=1}^{n} \mathbb{E}\left\{v_{n}^{-1} \Delta M_{k}(1)^{*}\left(V_{n}^{-1}(2) \Delta M_{k}(2)\right) / \mathfrak{F}_{k-1}\right\} \\
& =O\left(\sum_{k=1}^{n}\left[v_{k}^{-2} \operatorname{tr}\left(\Delta\langle M\rangle_{k}(1)\right)\right]^{1 / 2}\left\|V_{n}^{-1}(2) V_{k}(2)\right\|\right) \\
& =O\left(\sum_{k=1}^{n} k^{-\alpha / 2} \prod_{l=k}^{n-1}\left\|\Lambda_{l}\right\|\right)=O\left(n^{-\alpha / 2}\right)=o\left(n^{-\frac{1-\alpha}{2}}\right) \text { p.s. }
\end{aligned}
$$

c) Preuves des assertions 2) et 3) du théorème

D'après (5.58) et (5.59), on a :

$$
\zeta_{n}=o\left(n^{\frac{1-\alpha}{2}}\right)+O\left(\sum_{k=1}^{n} k^{-\alpha}\left\|V_{k}^{-1}\right\|^{2}\right)+O\left(\sum_{k=1}^{n} k^{-\alpha} k^{-\alpha / 2} \operatorname{tr}\left\{\widetilde{\mathfrak{D}}_{k}\right\}\right)=o\left(n^{\frac{1-\alpha}{2}}\right) \text { p.s. }
$$

car :

$$
\begin{aligned}
\operatorname{tr}\left\{\widetilde{\mathfrak{D}}_{k}\right\} & =O\left(\left\|V_{k}^{-1} M_{k}\right\|^{2}\right)=O\left(\left\|v_{k}^{-1} M_{k}(1)\right\|^{2}\right)+O\left(\left\|V_{k}^{-1}(2) M_{k}(2)\right\|^{2}\right) \\
& =O\left(\left(\log \log v_{k}^{2}\right)\right)+o\left(k^{\alpha-\frac{1}{2}}\right)=O((\log \log k))+o\left(k^{\alpha-\frac{1}{2}}\right)=o\left(k^{\alpha-\frac{1}{2}}\right) \text { p.s. }
\end{aligned}
$$

Compte tenu du lemme 5.7 et du fait que $\alpha>\frac{1}{2}$, on vérifie aisément que la martingale matricielle $\left(\sum_{k=0}^{n}(k+1)^{-\alpha}\right.$ $\left.\Delta\left(2 H_{k+1}(2)+\bar{H}_{k+1}(2)\right)\right)$ considérée dans (5.55) est p.s. convergente, car sa variation quadratique prévisible est p.s. convergente. Il en résulte, grâce à (5.57) et (5.60), que :

$$
\sum_{k=1}^{n} k^{-\alpha}\left({ }^{*} M_{k} Q_{k}^{-1} M_{k}-d\right)=\frac{2}{s^{2}} \operatorname{tr}\left\{C^{-1}(1) K_{n+1}(1)\right\}+o\left(n^{\frac{1-\alpha}{2}}\right) \text { p.s. }
$$

D'où la partie 3) du théorème, grâce au lemme 5.6 et à la partie j) de la section 5.2.2. 
On obtient la partie 2) en montrant par la méthode de la fonction caractéristique conditionnelle, comme au lemme 5.6, que :

$$
\left\{n^{-1 / 2} K_{n+1}(1), v_{n}^{-1} M_{n}(1), V_{n}^{-1}(2) M_{n}(2)\right\} \underset{n \rightarrow \infty}{\stackrel{\mathcal{L}}{\longrightarrow}} \mathfrak{N}_{d_{1} \times d_{1}}(0, C(1) \otimes C(1)) \otimes \mathfrak{N}_{d_{1}}(0, C(1)) \otimes \nu
$$

où $\nu$ est la loi de la v.a. $\Sigma_{2}(\eta)$, limite en loi de la suite $\left(V_{n}^{-1}(2) M_{n}(2)\right)$.

\section{AnNexe}

\section{GÉNÉRALISATION DU THÉORÈME DE LA LIMITE CENTRALE PRESQUE-SÛRE POUR LES MARTINGALES VECTORIELLES}

Les résultats suivants ont été établis dans $[5,6]$.

Théorème I ( $1^{\text {re }}$ forme du TLCPSG). Soit $M=\left(M_{n}\right)_{n \geq 0}$ une martingale à valeurs dans $\mathbb{R}^{d}$, localement de carré intégrable, satisfaisant aux hypothèses $H$-1) et H-2) avec une normalisation $\left(V_{n}\right)_{n>0}$ vérifiant ou bien les conditions $(\mathcal{C})$ (cas 1) ou bien les conditions $\left(\mathcal{C}^{\prime}\right)$ (cas 2). Soit $\left(r_{n}\right)_{n \geq 0}$ une suite de réels positifs majorée et telle que $R_{N}=\sum_{n=1}^{N} r_{n} \rightarrow \infty$. Considérons les mesures empiriques pondérées :

$$
\begin{gathered}
\mu_{N}^{1}(.)=\left[\log \left(\text { Dét } V_{N}\right)^{2}\right]^{-1} \sum_{n=1}^{N}\left[1-\left(\frac{\text { Dét } V_{n}}{\text { Dét } V_{n+1}}\right)^{2}\right] \delta_{\left\{Z_{n} \in .\right\}}, \\
\mu_{N}^{2}(.)=R_{N}^{-1} \sum_{n=1}^{N} r_{n} \delta_{\left\{Z_{n} \in .\right\}}
\end{gathered}
$$

associées aux v.a. $Z_{n}=V_{n}^{-1} M_{n}$. Alors, presque-sûrement, dans le cas $j,(j=1,2)$, les mesures $\left(\mu_{N}^{j}\right)$ convergent étroitement vers $\mu_{\infty}$.

Théorème II ( $2^{\mathrm{e}}$ forme du TLCPSG). Soit $M=\left(\left(M_{n}(1), M_{n}(2)\right)\right)_{n \geq 0}$ une martingale à valeurs dans $\mathbb{R}^{d_{1}} \times$ $\mathbb{R}^{d_{2}}$, localement de carré intégrable, satisfaisant aux hypothèses $H$-1) et $H$-2) avec une normalisation $\left(V_{n}\right)_{n \geq 0}$ de la forme : $V_{n}=\operatorname{Diag}\left(V_{n}(1), V_{n}(2)\right)$ où les matrices carrées $\left(V_{n}(1)\right)\left[\right.$ resp. $\left.\left(V_{n}(2)\right)\right]$ vérifient les conditions $(\mathcal{C})\left[\right.$ resp. $\left.\left(\mathcal{C}^{\prime}\right)\right]$. Associons aux v.a. $Z_{n}=\left(Z_{n}(1), Z_{n}(2)\right)$ avec $Z_{n}(i)=V_{n}^{-1}(i) M_{n}(i)$ les mesures empiriques pondérées:

$$
\mu_{N}(.)=\left[\log \text { Dét } V_{N}^{2}(1)\right]^{-1} \sum_{n=1}^{N}\left[1-\left(\frac{\text { Dét } V_{n}(1)}{\text { Dét } V_{n+1}(1)}\right)^{2}\right] \delta_{\left\{Z_{n} \in .\right\}} .
$$

Alors, presque-sûrement, les mesures $\left(\mu_{N}\right)$ convergent étroitement vers la mesure $\mu_{\infty}$.

Du théorème I, on déduit le corollaire suivant appelé théorème de limite centrale presque-sûre multidimensionnel (TLCPS) :

Corollaire III (TLCPS). Si dans le cas 1 du théorème I, l'hypothèse H-2) est remplacée par la condition de Lindeberg $\left.H^{\prime}-2\right)$, alors la limite $\mu_{\infty}(\omega, \bullet)$ des mesures $\mu_{N}^{1}(\omega, \bullet)$ est la loi mélange aléatoire de $C^{1 / 2}(\omega) G$ où $G$ est un vecteur gaussien d-dimensionnel, standard et indépendant de $C$. En particulier, $\mu_{\infty}=\mathfrak{N}(0, C)$ si en plus la matrice $C$ n'est pas aléatoire.

Comme conséquence du (TLCPSG), on obtient les minorations suivantes appelées, à juste titre, inégalités fortes de Cramer-Rao dans [5] et [6]. 


\section{Corollaire IV}

1) Sous les hypothèses du théorème II, et en supposant que la probabilité limite $\mu_{\infty}$ est de covariance $C$ (i.e. $\left.C=\int x^{*} x d \mu_{\infty}(x)\right)$, presque-sûrement pour tous vecteurs $u, v$ de $\mathbb{R}^{d}$ :

$$
\begin{aligned}
& \varliminf_{N \rightarrow \infty}\left(\log \left[\text { Dét } V_{N}(1)\right]^{2}\right)^{-1} \sum_{n=1}^{N}\left[1-\left(\frac{\text { Dét } V_{n}(1)}{\text { Dét } V_{n+1}(1)}\right)^{2}\right]\left|\left\langle u, V_{n}^{-1} M_{n}\right\rangle\left\langle v, V_{n}^{-1} M_{n}\right\rangle\right| \\
& \geq\left|{ }^{*} u C v\right| \text {; }
\end{aligned}
$$

en particulier :

$$
\varliminf_{N \rightarrow \infty}\left(\log \left[\text { Dét } V_{N}(1)\right]^{2}\right)^{-1} \sum_{n=1}^{N}\left[1-\left(\frac{\text { Dét } V_{n}(1)}{\text { Dét } V_{n+1}(1)}\right)^{2}\right]\left\|V_{n}^{-1} M_{n}\right\|^{2} \geq \operatorname{tr}(C) \text {. }
$$

2) Si la probabilité $\mu_{\infty}$ est de covariance $C$, alors dans le cadre 2 du théorème $I$ :

$$
\varliminf_{N \rightarrow \infty} \frac{1}{N} \sum_{n=1}^{N}\left|\left\langle u, V_{n}^{-1} M_{n}\right\rangle\left\langle v, V_{n}^{-1} M_{n}\right\rangle\right| \geq\left|{ }^{*} u C v\right|
$$

presque-sûrement pour tous vecteurs $u, v$ de $\mathbb{R}^{d}$.

Les auteurs tiennent à remercier l'éditeur et les deux rapporteurs pour leurs commentaires constructifs qui ont permis d'améliorer la première version de ce travail.

\section{REFERENCES}

[1] S. Asmussen et N. Keiding, Martingale central limit theorems and asymptotic theory for multitype branching processes. Adv. in Appl. Probab. 10 (1978) 109-129.

[2] K.B. Athreya et P.E. Ney, Branching processes. Springer, Berlin (1972).

[3] I. Berkes, L. Horvath et D. Khoshnevisan, Logarithmic averages of stable rondom variables are asymptotically normal. Stochastic Process. Appl. 77 (1998) 35-51.

[4] F. Chaâbane, Version forte du théorème de la limite centrale fonctionnel pour les martingales. C. R. Acad. Sci. Paris Sér. I. Math. 323 (1996) 195-198.

[5] F. Chaâbane, F. Maâouia et A. Touati, Généralisation du théorème de la limite centrale presque-sûr pour les martingales vectorielles. C. R. Acad. Sci. Paris Sér. I. Math. 326 (1998) 229-232.

[6] F. Chaâbane, F. Maâouia et A. Touati, Versions fortes des théorèmes limites en loi pour les martingales vectorielles (soumis).

[7] F. Chaâbane, Invariance principles with logarithmic averaging for martingales. Studia Sci. Math. Hungar (à paraître).

[8] F. Chaâbane, F. Maâouia et A. Touati, Théorèmes limites avec poids pour des modèles statistiques. Prépublication de la Faculté des Sciences de Bizerte (1998).

[9] F. Chaâbane et A. Touati, Sur la méthode de "moyennisation" logarithmique pour l'identification de modèles de régression linéaires. Prépublication de la Faculté des Sciences de Bizerte (2000).

[10] H.F. Chen et L. Guo, Identification and stochastic adaptive control. Birkhäuser (1991).

[11] M. Csörgö et L. Horvath, Invariance principles for logarithmic aerages. Process. Camb. Phil. Soc. (1992) 112-195.

[12] D. Dacunha-Castelle et M. Duflo, Probabilités et statistiques, tome 2. Masson (1983).

[13] M. Duflo, R. Senoussi et A. Touati, Sur la loi des grands nombres pour les martingales vectorielles et l'estimateur des moindres carrés d'un modèle de régression. Ann. Inst. H. Poincaré 26 (1990) 549-566.

[14] M. Duflo, Random iterative models. Springer-Verlag (1997).

[15] M. Duflo, Algorithmes Stochastiques. Springer-Verlag (1996).

[16] P. Hall et C.C. Heyde, Martingale limit theory and its applications. Academic Press (1981).

[17] J. Jacod et A.N. Shiryaev, Limit theorems for stochastic processes. Springer-Verlag (1987).

[18] L. Le Cam, Asymptotic methods in statistical decision theory. Springer-Verlag (1986).

[19] F. Maâouia, Versions fortes du théorème de la limite centrale pour les processus de Markov. C. R. Acad. Sci. Paris Sér. I. Math. 323 (1996) 293-296. 
[20] F. Maâouia, Principes d'invariance par "moyennisation" logarithmique pour les processus de Markov. Prépublication de la Faculté des Sciences de Bizerte (soumis).

[21] F. Maâouia et A. Touati, Identification of multitype branching processes. Prépublication de la Faculté des Sciences de Bizerte (1999).

[22] M. Pelletier, On the almost sure asymptotic behaviour of stochastic algorithms. Stochastic Process. Appl. 78 (1998) $217-244$.

[23] A. Touati, Two theorems on convergence in distribution for stochastic integrals and statistical applications. Probab. Th. Applications 38 (1993) 95-117.

[24] A. Touati, Sur les versions fortes du théorème de la limite centrale. Prépublication de l'Université de Marne-La-Vallée, $n^{\circ} 23$ (1995)

[25] A. Touati, Vitesse de convergence en loi de l'estimateur des moindres carrés d'un modèle autorégressif (cas mixte). Ann. Inst. H. Poincaré 32 (1996) 211-230.

[26] C.Z. Wei, Asymptotic properties of least-squares estimates in stochastic regression models. Ann. Stat. 13 (1985) $1498-1508$.

[27] C.Z. Wei, Adaptive prediction by least squares predictors in stochastic regression models with applications to time series. Ann. Statist. 15 (1987) 1667-1682. 